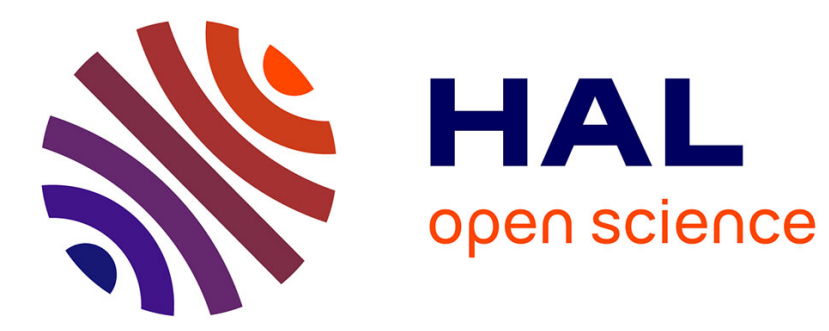

\title{
A highly anisotropic nonlinear elasticity model for vesicles. II. Derivation of the thin bilayer bending theory
}

Benoit Merlet

\section{To cite this version:}

Benoit Merlet. A highly anisotropic nonlinear elasticity model for vesicles. II. Derivation of the thin bilayer bending theory. 2013. hal-00848552v2

HAL Id: hal-00848552

https://hal.science/hal-00848552v2

Preprint submitted on 2 Sep 2014

HAL is a multi-disciplinary open access archive for the deposit and dissemination of scientific research documents, whether they are published or not. The documents may come from teaching and research institutions in France or abroad, or from public or private research centers.
L'archive ouverte pluridisciplinaire HAL, est destinée au dépôt et à la diffusion de documents scientifiques de niveau recherche, publiés ou non, émanant des établissements d'enseignement et de recherche français ou étrangers, des laboratoires publics ou privés. 


\title{
A highly anisotropic nonlinear elasticity model for vesicles II. Derivation of the thin bilayer bending theory
}

the date of receipt and acceptance should be inserted later

\begin{abstract}
We study the thin-shell limit of the nonlinear elasticity model for vesicles introduced in part I. We consider vesicles of width $2 \varepsilon \downarrow 0$ with elastic energy of order $\varepsilon^{3}$. In this regime, we show that the limit model is a bending theory for generalized hypersurfaces - namely, co-dimension one oriented varifolds without boundary. Up to a positive factor, the limit functional is the Willmore energy. In the language of $\Gamma$-convergence, we establish a compactness result, a lower bound result and the matching upper bound in the smooth case.
\end{abstract}

Keywords Calculus of Variation $\cdot \Gamma$-convergence $\cdot$ Willmore functional Rigidity estimates $\cdot$ Non-linear elasticity $\cdot$ Lipid bilayers

Mathematics Subject Classification (2000) 49Q10 - 49Q15 · 74B20 · 74K25 · $74 \mathrm{~K} 25$

\section{Contents}

1 Introduction .......................... 2

2 Compactness: approximation by hypersurfaces, proof of Theorem $1.1 \quad \ldots$. . . . 9

3 Construction of a recovery family in the smooth case. Proof of Theorem 1.3 . . . . 27

4 Lower bound. Proof of Theorem 1.2 . . . . . . . . . . . . . . . . . . . . . . . 29

5 Concluding remarks ......................... 45

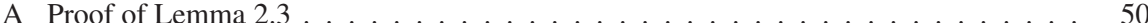

B Proofs of Lemmas 2.4, 2.5 and $2.6 \ldots \ldots \ldots$. . . . . . . . . . . . . . . 52

C Proof of Lemma 3.1 . . . . . . . . . . 56

D Oriented varifolds with $L^{2}$-generalized mean curvature . . . . . . . . . . . . . . . 57

Index of notation . . . . . . . . . . . . . . . . . . . . . . . 61

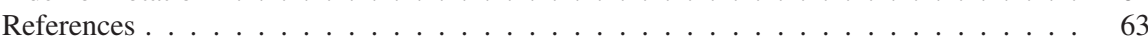

Benoît Merlet

Centre de Mathématiques Appliquées (CMAP), Ecole Polytechnique, 91128 Palaiseau, France.

E-mail: merlet@cmap.polytechnique.fr 


\section{Introduction}

In this article, we study the behavior as $\varepsilon$ goes to 0 of the nonlinear elasticity model for vesicle membranes with finite thickness introduced in [11]. More precisely, we perform a $\Gamma$-limit analysis of the family of functionals $\mathscr{F} / \varepsilon^{3}$ where $\varepsilon$ is the halfthickness of the membrane. The limit functional will be (up to a positive factor) the Willmore functional defined on generalized hypersufaces. Let us recall that the Willmore energy of a smooth compact hypersurface without boundary $\Sigma \subset \mathbf{R}^{d}$ is defined as

$$
\mathscr{W}(\Sigma):=\int_{\Sigma} h^{2} d \mathscr{H}^{d-1},
$$

where $h$ is the mean curvature of $\Sigma$.

This second part strongly depends on the first part of the paper. Before stating the main results, we recall the model, set some notation and introduce complementary assumptions. For simplicity, we specialize the model presented in [11] by assuming $\mu=0$ where $\mu$ is the parameter which accounts for the spontaneous curvature of the membrane. However, the general case is addressed in the last section.

\subsection{An Eulerian nonlinear elasticity model}

Let us fix an integer $d \geq 2$. Given $\varepsilon>0$, a membrane of thickness $2 \varepsilon$ in $\mathbf{R}^{d}$ is modeled by a bounded open set $\Omega \subset \mathbf{R}^{d}$ and two mappings $\tau \in L^{2}\left(\mathbf{R}^{d}, \mathbf{R}^{d}\right)$ and $\sigma \in L^{2}\left(\Omega, \mathbf{R}^{d}\right)$. These objects are subjected to a set of constraints: first, we assume that $\tau$ is a gradient vector field, more precisely, there exists $t \in W_{l o c}^{1,2}\left(\mathbf{R}^{d}\right) \cap$ $C\left(\mathbf{R}^{d},[-\varepsilon, \varepsilon]\right)$ such that $\tau=\nabla t$. Moreover, we assume

$$
\Omega=\left\{y \in \mathbf{R}^{d}:|t|(y)<\varepsilon\right\} .
$$

To prevent membranes from escaping to infinity, we fix a large radius $R>0$ and enforce

$$
|y|>R \quad \Longrightarrow \quad t(y)=+\varepsilon .
$$

Eventually, we assume that $\nabla \cdot \sigma=0$ in $\mathscr{D}^{\prime}(\Omega)$. Outside the set $\Omega$, we extend $\sigma$ by 0 ,

$$
\sigma(y):=0 \text { for every } y \in \mathbf{R}^{d} \backslash \Omega \text {. }
$$

We denote by $\mathscr{A}_{\varepsilon}(R)$ the set of triplets $(\sigma, \tau, \Omega)$ satisfying the above hypotheses and by $\mathscr{A}_{\varepsilon}$ the union $\cup_{R \uparrow_{\infty}} \mathscr{A}_{\varepsilon}(R)$.

The material density at some point $x \in \Omega$ is defined as $\sigma(x) \cdot \tau(x)$ and the total quantity of material is,

$$
\mathscr{Q}(\sigma, \tau):=\int_{\mathbf{R}^{d}} \sigma \cdot \tau .
$$

The parameter $2 \varepsilon$ should represent the thickness of the vesicle layer, hence the natural definition for the area of the membrane is $\mathscr{Q}(\sigma, \tau) / 2 \varepsilon$. Given a radius $R>0$ and a $(d-1)$-volume $S>0$, we set

$$
\mathscr{A}_{\varepsilon}(R, S):=\left\{(\sigma, \tau, \Omega) \in \mathscr{A}_{\varepsilon}(R): \mathscr{Q}(\sigma, \tau)=2 S \varepsilon\right\} .
$$


The elastic energy associated with a configuration $a=(\sigma, \tau, \Omega) \in \mathscr{A}_{\varepsilon}$ has the form

$$
\mathscr{F}(a):=\int_{\Omega} f(\sigma(y), \tau(y)) d y
$$

where $f \in C\left(\mathbf{R}^{d} \times \mathbf{R}^{d}, \mathbf{R}_{+}\right)$depends on the material. In our context, the storedenergy functions $f$ of interest vanish on the sphere

$$
\mathbb{S}^{d-1}:=\left\{(e, e): e \in S^{d-1}\right\} \subset \mathbf{R}^{d} \times \mathbf{R}^{d},
$$

that is

$$
f\left(\mathbb{S}^{d-1}\right)=\{0\} .
$$

For the lower bound part of the $\Gamma$-limit analysis, we also require that $f$ does not degenerate with respect to this constraint: we assume that the infimum of $f / d\left(\cdot, \mathbb{S}^{d-1}\right)^{2}$ over $\mathbf{R}^{d} \times \mathbf{R}^{d} \backslash \mathbb{S}^{d-1}$ is positive. Equivalently, we assume

$$
f \geq \kappa f_{0}, \quad \text { for some constant } \kappa>0,
$$

with $f_{0}(u, v):=|u-v|^{2}+(|u|-1)^{2}+(|v|-1)^{2}$, for every $u, v \in \mathbf{R}^{d}$.

The energy functional associated with this particular function is denoted by

$$
\mathscr{F}_{0}(\sigma, \tau, \Omega):=\int_{\Omega} f_{0}(\sigma(y), \tau(y)) d y .
$$

We study the $\Gamma$-limit as $\varepsilon$ tends to 0 of the energy $\mathscr{F} / \varepsilon^{3}$ defined on the set $\mathscr{A}_{\varepsilon}(R, S)$. For this we consider families $\left\{a_{\varepsilon}\right\}_{\varepsilon \in(0,1]}$ (or sequences $\left(a_{\varepsilon_{k}}\right)$ with $\varepsilon_{k} \downarrow 0$ ) of triplets $a_{\varepsilon}=\left(\sigma_{\varepsilon}, \nabla t_{\varepsilon}, \Omega_{\varepsilon}\right) \in \mathscr{A}_{\varepsilon}(R, S)$ with energy of order of $\varepsilon^{3}$ :

$$
\sup _{\varepsilon} \frac{\mathscr{F}\left(a_{\varepsilon}\right)}{\varepsilon^{3}}<\infty
$$

\subsection{Continuity hypothesis}

In the proofs, we need a uniform equicontinuity hypothesis which in general does not follow from (1.3) and might be only a technical assumption.

Hypothesis 1 There exists a modulus of continuity $\omega$ (i.e $\omega: \mathbf{R}_{+} \rightarrow \mathbf{R}_{+}$is a nondecreasing concave continuous function such that $\omega(0)=0$ ) such that

$$
\text { for every } \varepsilon \in(0,1], \quad t_{(\varepsilon)}: y \in \mathbf{R}^{d} \mapsto \frac{t_{\varepsilon}}{\varepsilon}(\varepsilon y) \text { is } \omega \text {-continuous. }
$$

Remark 1.1 The energy bound $\mathscr{F}_{0}(\sigma, \nabla t, \Omega)<\infty$ is not sufficient for $t$ being continuous. However, in the cases $d=2$ and $d=3$, if the stored energy function satisfies

$$
f(\sigma, \tau) \geq \kappa^{\prime}(|\tau|-1)^{p}
$$

for some $p>d$ and $\kappa^{\prime}>0$, then Hypothesis 1 is the consequence of the energy bound (1.3). Indeed, in this case,

$$
\int_{(1 / \varepsilon) \Omega_{\varepsilon}}\left(\left|\nabla t_{(\varepsilon)}\right|-1\right)^{p}=\varepsilon^{3-d}\left(\frac{1}{\varepsilon^{3}} \int_{\Omega_{\varepsilon}}\left(\left|\nabla t_{\varepsilon}\right|-1\right)^{p}\right) \leq\left(\sup _{\varepsilon} \frac{\mathscr{F}\left(a_{\varepsilon}\right)}{\varepsilon^{3}}\right) \frac{\varepsilon^{3-d}}{\kappa^{\prime}}
$$


Consequently, the family $t_{(\varepsilon)}$ has uniformly bounded gradients in $L^{p}\left(\mathbf{R}^{d}\right)$ and, by Morrey embedding theorem, is uniformly equi-Hölder-continuous with Hölder exponent $1-d / p$.

\subsection{Compactness}

Let us fix $R, S>0$ and consider a family $\left\{a_{\varepsilon}\right\}_{0<\varepsilon \leq 1}, a_{\varepsilon}=\left(\sigma_{\varepsilon}, \tau_{\varepsilon}, \Omega_{\varepsilon}\right) \in \mathscr{A}_{\varepsilon}(R, S)$, satisfying Hypothesis 1 and

$$
E_{0}:=\sup _{0<\varepsilon \leq 1} \frac{\mathscr{F}_{0}\left(a_{\varepsilon}\right)}{\varepsilon^{3}}<+\infty
$$

Our strategy is to approximate the membrane described by the data $a_{\varepsilon}$ by a smooth hypersurface $\Sigma_{\varepsilon}=\partial O_{\varepsilon}$ where $O_{\varepsilon}$ is an open subset of $B_{R}$ which is close in $L^{1}$ to $\left[t_{\varepsilon} \equiv-\varepsilon\right]$ - see Section 2, Proposition 2.1. We obtain uniform bounds on the $(d-1)$-volume of $\Sigma_{\varepsilon}$ and on the Wilmore energy $\mathscr{W}\left(\Sigma_{\varepsilon}\right)$. Sets of finite perimeter seem reasonable limit objects for the family $\left\{O_{\varepsilon}\right\}$ as up to extraction, $\left(O_{\varepsilon}\right)$ converges towards a set with finite perimeter $O_{0}$. Unfortunately, we may loose large pieces of membrane in the limit process: two (or more) pieces of the hypersurface $\Sigma_{\varepsilon}$ may coincide at the limit $\varepsilon \downarrow 0$, leading to $\mathscr{H}^{d-1}\left(\partial O_{0}\right)<S$. Moreover, if we consider the behavior of the Willmore energy, the limit surface $\Sigma_{0}=\partial O_{0}$ may not have square integrable mean curvature, as cusps arise on the boundary of the cancelling pieces of hypersurface (see Figure 1.1). To keep track of these phenomena and prevent cancellation, we have to take into account multiplicity. We do this by considering hypersurfaces as $(d-1)$-dimensional varifolds. Varifolds have been introduced as a generalization of manifolds by Almgren [2] for the study of Plateau's problem, (see also Allard [1] and the reference book by Simon [13]). More precisely, here we consider the set of oriented $(d-1)$-varifolds as introduced by Hutchinson [9].
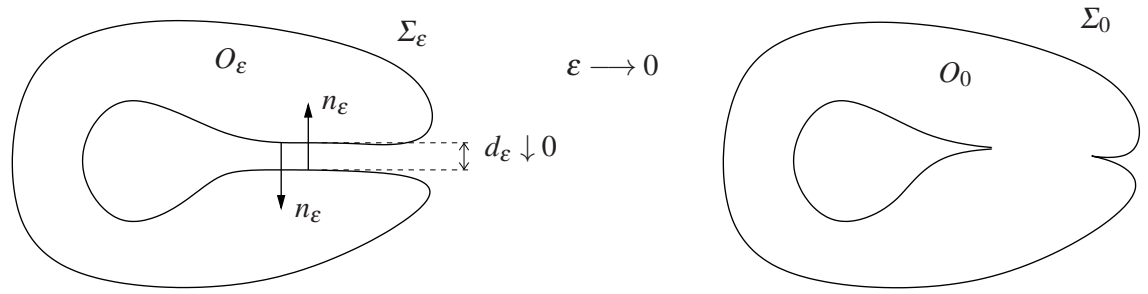

Fig. 1.1 Cancellation of boundaries with opposite orientations.

\section{Definition 1.1}

a) The space of oriented $(d-1)$-varifolds in $\mathbf{R}^{d}$ is the topological dual of $C_{c}\left(\mathbf{R}^{d} \times S^{d-1}\right)$, that is the space $\mathscr{M}\left(\mathbf{R}^{d} \times S^{d-1}\right)$ of Radon measures on $\mathbf{R}^{d} \times S^{d-1}$ endowed with the weak star topology of Radon measures. 
b) To any oriented $(d-1)$-varifold $\mathscr{V} \in \mathscr{M}\left(\mathbf{R}^{d} \times S^{d-1}\right)$, we associate a distribution $\Lambda \mathscr{V} \in \mathscr{D}^{\prime}\left(\mathbf{R}^{d}, \mathbf{R}^{d}\right)$ defined by

$$
\langle\Lambda \mathscr{V} ; \psi\rangle:=\langle\mathscr{V} ;(y, n) \mapsto \psi(y) \cdot n\rangle, \quad \text { for } \psi \in \mathscr{D}\left(\mathbf{R}^{d}, \mathbf{R}^{d}\right) .
$$

c) Given a smooth hypersurface $\Sigma$ oriented by $v$, we define the oriented $(d-$ 1)-varifold $\mathscr{V}=\mathscr{V}(\Sigma, v)$ by

$$
\langle\mathscr{V} ; \varphi\rangle:=\int_{\Sigma} \varphi(x, v(x)) d \mathscr{H}^{d-1}(x), \quad \text { for every } \varphi \in C_{c}\left(\mathbf{R}^{d} \times S^{d-1}\right) .
$$

Remark 1.2

a) Usually, oriented $k$-varifolds are defined as the Radon measures over $\mathbf{R}^{d} \times$ $G^{o}(k, d)$, where $G^{o}(k, d)$ denotes the Grassmannian of oriented $k$-subspaces of $\mathbf{R}^{d}$. Here, we consider varifolds with co-dimension 1 and we can identify any $(d-1)$ dimensional oriented subspace of $\mathbf{R}^{d}$ with its positively oriented unit normal. This defines a smooth diffeomorphism $G^{o}(d-1, d) \stackrel{\sim}{\rightarrow} S^{d-1}$.

b) Similarly, in the literature (see e.g. [9]), one associates with any oriented $k$-varifold a $k$-current $\mathscr{C} \mathscr{V}$. Here, we choose not to treat currents explicitly. We identify simple $(d-1)$ vectors of the form $(-1)^{i+1} e^{1} \wedge \cdots \wedge e^{i-1} \wedge e^{i+1} \wedge \cdots \wedge e^{d}$ with $e_{i}$. By duality, this identifies the $(d-1)$-current $\mathscr{C} \mathscr{V}$ with $\Lambda \mathscr{V}$. In fact, in the present paper the boundary of $\mathscr{C} \mathscr{V}$ always vanishes. With our notation, this amounts to saying that $\Lambda \mathscr{V}$ satisfies the Poincaré conditions $\partial_{i}[\Lambda \mathscr{V}]_{j}=\partial_{j}[\Lambda \mathscr{V}]_{i}$ for $i, j \in\{1, \cdots, d\}$ or equivalently that $\Lambda \mathscr{V}$ is a gradient.

In general the distribution $\Lambda \mathscr{V}$ carries strictly less information than the oriented varifold $\mathscr{V}$. For instance, if $\Sigma \neq \emptyset$ is a smooth compact hypersurface oriented by $v$ then $\mathscr{V}:=\mathscr{V}(\Sigma, v)+\mathscr{V}(\Sigma,-v)$ does not vanish but $\Lambda \mathscr{V} \equiv 0$.

c) When $\Sigma$ is the boundary of a smooth and bounded open set $O \subset \mathbf{R}^{d}$, with outward unit normal $v$, then $\Lambda[\mathscr{V}(\Sigma, v)]=-\nabla \mathbf{1}_{O}$.

Our compactness result concerns varifolds constructed from elements of $\mathscr{A}_{\varepsilon}$.

Definition 1.2 Let us fix a cut-off function $\chi^{\star} \in C_{c}^{\infty}(1 / 2,2)$ satisfying $\chi^{\star}(1)=1$. For $\varepsilon \in(0,1]$, we associate with any element $a=(\sigma, \nabla t, \Omega)$ of $\mathscr{A}_{\varepsilon}$ the oriented varifold,

$$
\left\langle\mathscr{V}_{\varepsilon}^{\star}(a) ; \varphi\right\rangle:=\frac{1}{2 \varepsilon} \int_{\Omega} \chi^{\star}(|\nabla t|(y)) \varphi\left(y, \frac{\nabla t}{|\nabla t|}(y)\right) d y, \quad \forall \varphi \in C\left(\mathbf{R}^{d} \times S^{d-1}\right) .
$$

Definition 1.3 (Limit set)

The limit set $\mathscr{A}_{0}(R, S)$ in our $\Gamma$-convergence analysis is a set of oriented $(d-$ 1)-varifolds. Namely, $\mathscr{V}_{0} \in \mathscr{M}\left(\mathbf{R}^{d} \times S^{d-1}\right)$ belongs to $\mathscr{A}_{0}(R, S)$ if there exists a sequence of smooth open sets $\left(O_{k}\right)_{k \geq 1} \subset B_{R}$ with outward unit normals $v_{k}$ and boundaries $\Sigma_{k}$ such that

$$
\begin{array}{ll}
\sup _{k} \mathscr{W}\left(\Sigma_{k}\right)<\infty, & \mathscr{H}^{d-1}\left(\Sigma_{k}\right) \stackrel{k \uparrow \infty}{\longrightarrow} S, \\
\text { and } & \mathscr{V}\left(\Sigma_{k}, v_{k}\right) \stackrel{k \uparrow \infty}{\longrightarrow} \mathscr{V}_{0} \text { as Radon measures. }
\end{array}
$$


We postpone further definitions and statements about varifolds to Appendix D. Let us say however that the elements of $\mathscr{A}_{0}(R, S)$ are oriented integer rectifiable $(d-1)$-varifolds (Definition D.1) which admit a $L^{2}$-generalized mean curvature (Definition D.2). The definition of the Willmore energy extends to these objects as a lower semi-continuous functional on $\mathscr{A}_{0}(R, S)$.

\section{Theorem 1.1}

Let $R, S>0$ and let $\left\{a_{\varepsilon}\right\}_{0<\varepsilon \leq 1}$ be a family of configurations $a_{\varepsilon}=\left(\sigma_{\varepsilon}, \nabla t_{\varepsilon}, \Omega_{\varepsilon}\right) \in$ $\mathscr{A}_{\varepsilon}(R, S)$ satisfying the energy bound (1.4) and Hypothesis 1. Then:

a) There exists a non-negative oriented $(d-1)$-varifold $\mathscr{V}_{0}$ with total mass $S$ such that, up to extraction,

$$
\mathscr{V}_{\varepsilon}^{\star}\left(a_{\varepsilon}\right) \stackrel{\varepsilon \downarrow 0}{\longrightarrow} \mathscr{V}_{0} \text { as Radon measures. }
$$

b) There exists a set of finite perimeter $O_{0} \subset B_{R}$, such that

$$
\nabla \mathbf{1}_{O_{0}}=-\Lambda \mathscr{V}_{0}, \quad \text { and } \quad T_{\varepsilon}:=\frac{\varepsilon-t_{\varepsilon}}{2 \varepsilon} \stackrel{\varepsilon \downarrow 0}{\longrightarrow} \mathbf{1}_{O_{0}} \text { weakly in } B V\left(\mathbf{R}^{d}\right) .
$$

c) Moreover, $\mathscr{V}_{0} \in \mathscr{A}_{0}(R, S)$ (hence $\mathscr{V}_{0}$ has finite generalized Willmore energy).

\section{Remark 1.3}

$i$. As a consequence of (b), if the interior domain $M_{\varepsilon}=\left[t_{\varepsilon} \equiv-\varepsilon\right]$ has prescribed volume or merely satisfies $\mathscr{H}^{d}\left(M_{\varepsilon}\right) \rightarrow V$, then $\mathscr{H}^{d}\left(O_{0}\right)=V$.

ii. The varifold $\mathscr{V}_{0}$ can be described by means of a $(d-1)$-rectifiable set $\Sigma_{0}$ and multiplicity functions $\theta_{0}^{ \pm}$. The Willmore energy $\mathscr{W}\left(\mathscr{V}_{0}\right)$ has an explicit expression in terms of the generalized curvature of $\Sigma_{0}$ and $\theta_{0}^{ \pm}$(see Definition D.1, Definition D.2 and formula (D.9)).

\subsection{Lower bound}

For the lower bound, we make further assumptions on the stored energy function. Namely, we assume that

$$
f \text { is of class } C^{2} \text { in some neighborhood } \mathscr{N} \text { of } \mathbb{S}^{d-1} \text { in } \mathbf{R}^{d} \times \mathbf{R}^{d},
$$

and is isotropic in this neighborhood, that is

$$
f(Q \sigma, Q \tau)=f(\sigma, \tau) \quad \forall Q \in \operatorname{SO}(d), \forall(\sigma, \tau) \in \mathscr{N} .
$$

This assumption parallels the frame indifference hypothesis in nonlinear elasticity.

Theorem 1.2 Let $R, S>0$ and let $\left(a_{\varepsilon_{k}}\right)_{\varepsilon_{k} \downarrow 0}$ with $a_{\varepsilon_{k}} \in \mathscr{A}_{\varepsilon_{k}}(R, S)$ be a sequence of configurations satisfying Hypothesis 1. Assume that there exists a $(d-1)$-varifold $\mathscr{V}_{0}$ such that $\mathscr{V}_{\varepsilon_{k}}^{\star}\left(a_{\varepsilon_{k}}\right) \rightarrow \mathscr{V}_{0}$ as Radon measures.

Then, for every $f \in C\left(\mathbf{R}^{d} \times \mathbf{R}^{d}, \mathbf{R}_{+}\right)$satisfying (1.1), (1.2), (1.6) and (1.7), we have

$$
c_{0}(f) \mathscr{W}\left(\mathscr{V}_{0}\right) \leq \liminf _{\varepsilon_{k} \downarrow 0} \frac{\mathscr{F}\left(a_{\varepsilon_{k}}\right)}{\varepsilon_{k}^{3}},
$$


where $c_{0}(f)>0$ only depends on the Hessian matrix $D^{2} f$ on $\mathbb{S}^{d-1}$. Namely,

$$
c_{0}(f):=\frac{\operatorname{det} L}{3 L_{2,2}} \quad \text { with } \quad L:=\left(\begin{array}{cc}
\frac{\partial^{2} f}{\partial \sigma_{d}^{2}} & \frac{\partial^{2} f}{\partial \sigma_{d} \partial \tau_{d}} \\
\frac{\partial^{2} f}{\partial \sigma_{d} \partial \tau_{d}} & \frac{\partial^{2} f}{\partial \tau_{d}^{2}}
\end{array}\right)\left(e_{d}, e_{d}\right) .
$$

1.5 Upper bound in the smooth case

Theorem 1.3 Let $f \in C\left(\mathbf{R}^{d} \times \mathbf{R}^{d}, \mathbf{R}_{+}\right)$satisfying (1.1), (1.6) and (1.7) and let $O_{0} \subset \mathbf{R}^{d}$ be a smooth bounded open subset with boundary $\Sigma_{0}$ and outward unit normal $v_{0}$. Let us define

$$
R:=\sup \left\{|y|: y \in O_{0}\right\}+1 \quad \text { and } \quad S:=\mathscr{H}^{d-1}\left(\Sigma_{0}\right) .
$$

Then there exists a family $\left\{a_{\varepsilon}\right\}_{0<\varepsilon \leq 1}, a_{\varepsilon} \in \mathscr{A}_{\mathcal{E}}(R, S)$, such that

$$
\mathscr{V}_{\varepsilon}^{\star}\left(a_{\varepsilon}\right) \stackrel{\varepsilon \downarrow 0}{\longrightarrow} \mathscr{V}\left(\Sigma_{0}, v_{0}\right) \text { as Radon measures and } c_{0}(f) \mathscr{W}\left(\Sigma_{0}\right)=\lim _{\varepsilon \downarrow 0} \frac{\mathscr{F}\left(a_{\varepsilon}\right)}{\varepsilon^{3}} \text {. }
$$

Moreover, noting $a_{\varepsilon}=\left\{\sigma_{\varepsilon}, \nabla t_{\varepsilon}, \Omega_{\varepsilon}\right\}$, the open sets $\Omega_{\varepsilon}$ and the vector fields $\sigma_{\varepsilon}$ and $\nabla t_{\varepsilon}$ are uniformly smooth. In particular Hypothesis 1 holds.

We expect the above upper bound to hold true for any element of the limit set $\mathscr{A}_{0}(R, S)$ and not only for smooth compact oriented manifolds, however the construction of a recovery family in the general case is a seemingly difficult open problem, see Section 5.

\subsection{Notation}

We present here the main conventions adopted in this work. The reader will find a list of notation in lexicographic order at the end of the article

Throughout the paper, the letter $C$ denotes a non-negative constant which is either a universal constant or only depends on the dimension $d$. For constants which also depend on other parameters, $\alpha_{1}, \cdots, \alpha_{k}$, we write $C\left(\alpha_{1}, \cdots, \alpha_{k}\right)$. As usual, the values of these constants may change from line to line. For constants which depend on the data introduced in the hypotheses (the dimension $d$, the prescribed $(d-1)$-volume $S$, the modulus of continuity $\omega$, the cut-off function $\chi^{\star}$ or the energy upper bound $E_{0}$ ) but not on $\varepsilon$, we use the short hand $C_{\varepsilon}$ and we write $C_{q}\left(\alpha_{1}, \cdots, \alpha_{k}\right)$ for constants also depending on other parameters. This convention extends to other objects which only depend on $\omega$ and $d$, namely: the modulus of continuity $\omega_{\mathscr{\varepsilon}}^{\star}$ of Lemma 2.3, the positive number $\delta_{\dot{q}}^{\star}$ of Corollary 2.1 and the functions $\beta_{\not, 1}^{\star}, \beta_{\not, 2}^{\star}, \beta_{\dot{q}, 3}^{\star}$ introduced in Lemmas 2.4, 2.5, 2.6.

We write $B_{r}(y)$ to denote the open ball in $\mathbf{R}^{d}$ with center $y$ and radius $r>0$ or simply $B_{r}$ for $B_{r}(0)$. 
The $(d-1)$-dimensional unit sphere of $\mathbf{R}^{d}$ is denoted by $S^{d-1}$.

The $k$-dimensional Hausdorff measure of a set $E \subset \mathbf{R}^{d}$ is denoted by $\mathscr{H}^{k}(E)$.

We often define the set of elements satisfying a property $\mathscr{P}$ by $[\mathscr{P}]$. For instance $[t=\varepsilon]$ is the set $\left\{y \in \mathbf{R}^{d}: t(y)=\varepsilon\right\}$.

Most of the time, we use $y$ or $z$ to denote a generic element of $\mathbf{R}^{d}$ whereas $x$ is always a point on a hypersurface.

For $e \in S^{d-1}, \pi_{e}$ denotes the orthogonal projection on the space $e^{\perp}=\left\{y \in \mathbf{R}^{d-1}\right.$ : $y \cdot e=0\}$, that is $\pi_{e}(y)=y-(y \cdot e) e$.

We identify $e_{d}^{\perp}$ with $\mathbf{R}^{d-1}$ and for $y \in \mathbf{R}^{d}$, we write $y^{\prime}=\left(y_{1}, \cdots, y_{d-1}\right)=\pi_{e_{d}} y$, so that $y=\left(y^{\prime}, y_{d}\right)$.

Some objects introduced along the proofs are used in different and sometimes distant parts of the paper. These objects are singled out by means of a superscript star: $\omega_{\tilde{\xi}}^{\star}, U^{\star}$, etc. We have already met the cut-off function $\chi^{\star}$ in Definition 1.2.

We use the prefix "I" to refer to a result of the first part of this article. For instance, Theorem 2.1 in [11] is refered as Theorem I.2.1.

\subsection{Outline of the paper}

In Section 2, we establish the compactness result Theorem 1.1. The main part of this section is devoted to the proof of Proposition 2.1 which contains the relevant constructions and estimates. In particular, we use the rigidity estimates of [11] to show that we can approximate the varifolds $\left\{\mathscr{V}^{\star}\left(a_{\varepsilon}\right)\right\}_{\varepsilon}$ by oriented hypersurfaces $\left\{\left(\Sigma_{\varepsilon}, v_{\varepsilon}\right)\right\}_{\varepsilon}$ with uniformly bounded Willmore energy.

In Section 2.6, we establish Theorem 1.1 as a consequence of Proposition 2.1 and of Allard's compactness theorem for integer rectifiable varifolds applied to the family of oriented hypersurfaces $\left\{\left(\Sigma_{\varepsilon}, v_{\varepsilon}\right)\right\}_{\varepsilon}$.

In Section 3, we build a recovery sequence in the smooth case, proving Theorem 1.3. We also describe there the general form of the Hessian matrix of our anisotropic stored energy functions on the set $\mathbb{S}^{d-1}$.

Section 4 is devoted to the proof of the lower bound of Theorem 1.2. In Section 4.1, we introduce an approximate mean curvature at the $\varepsilon$-level. At some point $x \in \Sigma_{\varepsilon}$, this approximate mean curvature only depends on the restriction of $\sigma_{\varepsilon}$ to the ball $B_{\sqrt{2} \varepsilon}(x)$. We show that this approximate curvature is indeed an approximation of the mean curvature on $\Sigma_{\varepsilon}$ in a weak sense. Again, the rigidity estimates are crucial in this step. In Section 4.2 we pass to the limit $\varepsilon \downarrow 0$ using lower semicontinuity of the Willmore energy. This reduces the lower bound problem to a relatively easy local optimization problem: minimize the local energy under prescribed approximate mean curvature.

In Section 5, we discuss the hypotheses and indicate possible generalizations and open problems. In particular, we consider the case of a material with spontaneous curvature $\mu \neq 0$ by substituting the condition $\nabla \cdot\left[\left(\rho\left(\mu t_{\varepsilon}\right)\right)^{-1} \sigma_{\varepsilon}\right] \equiv 0$ for the condition $\nabla \cdot \sigma_{\varepsilon} \equiv 0$.

In Appendices A, B and C we prove some ancillary results stated in the main text. In Appendix D we introduce further material concerning varifolds. The aim 
of the latter is to provide a better understanding of the limit set $\mathscr{L}_{0}(R, S)$ and to define the Willmore energy of generalized hypersurfaces.

\section{Compactness: approximation by hypersurfaces, proof of Theorem 1.1}

Let $R, S>0$ and let us consider a family $\left\{a_{\varepsilon}\right\}_{0<\varepsilon \leq 1}$ satisfying the hypotheses of Theorem 2.1. In this section, for every $\varepsilon$, we build a smooth open set $O_{\varepsilon}$ with boundary $\Sigma_{\varepsilon}$ and outward unit normal $v_{\varepsilon}$ such that $O_{\varepsilon}$ is close in $L^{1}$ to $\left[t_{\varepsilon} \equiv-\varepsilon\right]$ and $\mathscr{V}\left(\Sigma_{\varepsilon}, v_{\varepsilon}\right)$ is close to $\mathscr{V}_{\varepsilon}^{\star}\left(a_{\varepsilon}\right)$. The relevant uniform bounds and properties of these families are collected in Proposition 2.1 below whose proof occupies Sections 2.1 to 2.5. We deduce Theorem 1.1 from the proposition in Section 2.6.

\section{Proposition 2.1}

a) For every $\varepsilon \in(0,1]$,

$$
\left|\frac{1}{2 \varepsilon} \mathscr{H}^{d}\left(\Omega_{\varepsilon}\right)-S\right|+\left|\frac{1}{2 \varepsilon} \int_{\mathbf{R}^{d}}\right| \nabla t_{\varepsilon}|-S|+\left|\left\langle\mathscr{V}_{\varepsilon}^{\star}\left(a_{\varepsilon}\right) ; 1\right\rangle-S\right| \leq C_{\not{q}} \varepsilon .
$$

b) For every $\varepsilon \in(0,1]$, there exists a smooth bounded open set $O_{\varepsilon} \subset B_{R+C \varepsilon}$ with outward unit normal $v_{\varepsilon}$, such that, using the notation $\Sigma_{\varepsilon}:=\partial O_{\varepsilon}$ and $M_{\varepsilon}:=$ $\left\{y \in \mathbf{R}^{d}: t_{\varepsilon}(y)=-\varepsilon\right\}$, we have

$$
\left\|\mathbf{1}_{O_{\varepsilon}}-\mathbf{1}_{M_{\varepsilon}}\right\|_{L^{1}} \leq C_{\not \varepsilon} \varepsilon, \quad \mathscr{H}^{d-1}\left(\Sigma_{\varepsilon}\right) \leq C_{\mathscr{\xi}}, \quad \mathscr{W}\left(\Sigma_{\varepsilon}\right) \leq C_{\not \notin} .
$$

c) For every $\varphi \in C\left(\mathbf{R}^{d} \times \mathbf{R}^{d}\right)$ such that $\sup _{y, n}|\varphi(y, n)| /\left(1+|n|^{2}\right)<\infty$, we have

$$
\left|\frac{1}{2 \varepsilon} \int_{\Omega_{\varepsilon}} \varphi\left(y, \nabla t_{\varepsilon}(y)\right) d y-\int_{\Sigma_{\varepsilon}} \varphi\left(x, v_{\varepsilon}(x)\right) d \mathscr{H}^{d-1}(x)\right| \stackrel{\varepsilon \downarrow 0}{\longrightarrow} 0 .
$$

Except in the proof of part (c) of the proposition (Section 2.5), the parameter $\varepsilon \in(0,1]$ is fixed. In this case, it is convenient to rescale the domain by a factor $1 / \varepsilon$ by setting $\Omega_{(\varepsilon)}:=\varepsilon^{-1} \Omega_{\varepsilon}$. We also define the rescaled data:

$t_{(\varepsilon)}(y):=\varepsilon^{-1} t_{\varepsilon}(\varepsilon y), \quad \tau_{(\varepsilon)}(y):=\nabla t_{\varepsilon}(\varepsilon y), \quad \sigma_{(\varepsilon)}(y):=\sigma_{\varepsilon}(\varepsilon y) \quad$ for $y \in \mathbf{R}^{d}$.

With this notation, the function $t_{(\varepsilon)} \in C\left(\mathbf{R}^{d},[-1,1]\right)$ is $\omega$-continuous, we have $\nabla t_{(\varepsilon)}=\tau_{(\varepsilon)}$ and $\Omega_{(\varepsilon)}=\left\{y \in \mathbf{R}^{d}:\left|t_{(\varepsilon)}\right|(y)<1\right\}$. The vector field $\sigma_{(\varepsilon)}$ is divergence free in $\Omega_{(\varepsilon)}$ and vanishes in $\mathbf{R}^{d} \backslash \Omega_{(\varepsilon)}$. We also have,

$$
\begin{gathered}
\mathscr{Q}\left(\sigma_{(\varepsilon)}, \nabla t_{(\varepsilon)}\right)=\int_{\mathbf{R}^{d}} \sigma_{(\varepsilon)} \cdot \nabla t_{(\varepsilon)}=2 S / \varepsilon^{d-1}, \\
\text { and } \quad \mathscr{F}_{0}\left(\sigma_{(\varepsilon)}, \nabla t_{(\varepsilon)}, \Omega_{(\varepsilon)}\right) \leq E_{0} / \varepsilon^{d-3}
\end{gathered}
$$

Part (a) is established in Section 2.1. In Section 2.2, we introduce the harmonic extension $u_{(\varepsilon)}$ of $t_{(\varepsilon)}$ in a subset of $\Omega_{(\varepsilon)}$ which contains $\left[\left|t_{(\varepsilon)}\right|<4 / 5\right]$. The level sets of $u_{(\varepsilon)}$ are candidates for the hypersurface $\Sigma_{(\varepsilon)}$.

In Section 2.3 we state and prove some technical lemmas which follow from the weak rigidity estimates of [11]. These lemmas are designed for selecting the 
"good" points where the data is sufficiently close to a zero energy limit state, as described in Theorem I.1.1. We also state there all the consequences of the strong rigidity estimates which are relevant to our purpose. Eventually, we bound the volume of "bad" regions. All these results are also used in the proof of the lower bound in Section 4.

In Section 2.4, we build the set $O_{(\varepsilon)}$ (and therefore the hypersurface $\Sigma_{(\varepsilon)}$ ) and we prove the estimates of part (b). Eventually, the convergence result (c) is established in Section 2.5.

To lighten notation, we drop all the subscripts $(\varepsilon)$ and write $t$ for $t_{(\varepsilon)}, \sigma$ for $\sigma_{(\varepsilon)}, \Omega$ for $\Omega_{(\varepsilon)}$, etc. We come back to the unambiguous notation at the beginning of Section 2.5 when considering the limit $\varepsilon \downarrow 0$.

\subsection{Proof of Proposition 2.1. a}

For $-1 \leq \alpha_{-}<\alpha_{+} \leq 1$, we set

$$
\Omega_{\alpha_{-}}^{\alpha_{+}}:=\left\{y \in \mathbf{R}^{d}: \alpha_{-}<t(y)<\alpha_{+}\right\} \subset \Omega .
$$

Lemma 2.1 Let $-1 \leq \alpha_{-}<\alpha_{+} \leq 1$, we have

$$
\int_{\Omega_{\alpha_{-}}^{\alpha_{+}}} \nabla t \cdot \sigma=\left(\alpha_{+}-\alpha_{-}\right) S / \varepsilon^{d-1} .
$$

Moreover,

$\left|\mathscr{H}^{d}\left(\Omega_{\alpha_{-}}^{\alpha_{+}}\right)-\frac{\left(\alpha_{+}-\alpha_{-}\right) S}{\varepsilon^{d-1}}\right| \leq \frac{5}{2} \mathscr{F}_{0}\left(\sigma, \nabla t, \Omega_{\alpha_{-}}^{\alpha_{+}}\right)+2 \sqrt{\frac{S \mathscr{F}_{0}\left(\sigma, \nabla t, \Omega_{\alpha_{-}}^{\alpha_{+}}\right)}{\varepsilon^{d-1}}}$,

and similarly,

$$
\left|\int_{\Omega_{\alpha_{-}}^{\alpha_{+}}}\right| \nabla t\left|-\frac{\left(\alpha_{+}-\alpha_{-}\right) S}{\varepsilon^{d-1}}\right| \leq 4 \mathscr{F}_{0}\left(\sigma, \nabla t, \Omega_{\alpha_{-}}^{\alpha_{+}}\right)+4 \sqrt{\frac{S \mathscr{F}_{0}\left(\sigma, \nabla t, \Omega_{\alpha_{-}}^{\alpha_{+}}\right)}{\varepsilon^{d-1}}} .
$$

Before proving the lemma let us show that it implies Proposition 2.1.a. Applying (2.3) and (2.4) with $\alpha_{ \pm}= \pm 1$, unscaling and taking into account (2.1), we obtain

$$
\left|\frac{1}{2 \varepsilon} \mathscr{H}^{d}\left(\Omega_{\varepsilon}\right)-S\right|+\left|\frac{1}{2 \varepsilon} \int_{\mathbf{R}^{d}}\right| \nabla t_{\varepsilon}|-S| \leq C_{\not \varepsilon} \varepsilon
$$

Next, we have

$$
\left\langle\mathscr{V}_{\varepsilon}^{\star}\left(a_{\varepsilon}\right) ; 1\right\rangle=\frac{1}{2 \varepsilon} \int_{\Omega_{\varepsilon}} \chi^{\star}\left(\left|\nabla t_{\varepsilon}\right|(y)\right) d y .
$$

Since $\left|\chi^{\star}(\tau)-1\right| \leq C|| \tau|-1|$, we get

$$
\begin{aligned}
&\left|\left\langle\mathscr{V}_{\varepsilon}^{\star}\left(a_{\varepsilon}\right) ; 1\right\rangle-S\right| \leq \frac{C}{2 \varepsilon} \int_{\Omega_{\varepsilon}}|| \nabla t_{\varepsilon}|-1|+\left|\frac{1}{2 \varepsilon} \mathscr{H}^{d}\left(\Omega_{\varepsilon}\right)-S\right| \\
& \stackrel{(1.4),(2.5)}{\leq} C \sqrt{\mathscr{H}^{d}\left(\Omega_{\varepsilon}\right) / 2 \varepsilon} \sqrt{E_{0}} \varepsilon+C_{\not d} \varepsilon \stackrel{(2.5)}{\leq} C_{\not d} \varepsilon .
\end{aligned}
$$

Proposition 2.1.a follows from (2.5),(2.6). 
Proof (of Lemma 2.1) Let us first establish that for every smooth open set $O$ such that

$$
\left\{x \in \mathbf{R}^{d}: t(x)>-1\right\} \subset O \subset\left\{x \in \mathbf{R}^{d}: t(x)<1\right\},
$$

we have

$$
\int_{\partial o} \sigma \cdot v=S / \varepsilon^{d-1}
$$

where $v$ denotes the outward unit normal on $\partial O$ and $\sigma \cdot v$ is well defined in $H^{-1 / 2}(\partial O)$ as the trace on $\partial O$ of the normal component of $\sigma \in H_{\text {div }}(\Omega)=\left\{\sigma^{\prime} \in\right.$ $\left.L^{2}\left(\Omega, \mathbf{R}^{d}\right): \operatorname{div} \sigma^{\prime} \in L^{2}(\Omega)\right\}$.

Now, notice that the identity

$$
\int_{O} \nabla \varphi \cdot \sigma=\int_{\partial O} \varphi \sigma \cdot v
$$

is valid for every $\varphi \in C^{\infty}(\bar{\Omega})$ such that $\varphi \equiv 0$ in the neighborhood of $[t=-1]$. In order to extend this formula to the case $\varphi=t+1$, we introduce for $s \in(0,1)$, the truncated function $t_{s}$ defined as

$$
t_{s}(y):=\left\{\begin{array}{cl}
t(y) & \text { if }|t(y)| \leq 1-s \\
\pm(1-s) & \text { if } \pm t(y)>1-s
\end{array}\right.
$$

By continuity of $t$, the function $\varphi_{s}:=t_{s}+(1-s) \in W^{1,2}(\Omega)$ vanishes in the neighborhood of $[t=-1]$. Consequently, $\varphi_{s}$ belongs to the closure in $W^{1,2}$ of $\left.\left\{\varphi \in C^{\infty}(\bar{\Omega}): \operatorname{supp} \varphi \cap[t \leq-1]\right\}=\emptyset\right\}$ and (2.8) is valid with $\varphi=\varphi_{s}$.

$$
\int_{O} \nabla t_{s} \cdot \sigma=\int_{\partial O}\left(t_{s}+(1-s)\right) \sigma \cdot v .
$$

Letting $s \downarrow 0$, since $t_{s} \rightarrow t$ in $W_{l o c}^{1,2}\left(\mathbf{R}^{d}\right)$, we obtain,

$$
\int_{O} \nabla t \cdot \sigma=\int_{\partial O}(t+1) \sigma \cdot v .
$$

Similarly, integrating $\nabla\left(t_{s}-(1-s)\right) \cdot \sigma$ on $\Omega \backslash \bar{O}$ and passing to the limit $s \downarrow 0$, we also get,

$$
\int_{\Omega \backslash \bar{O}} \nabla t \cdot \sigma=\int_{\partial O}(-t+1) \sigma \cdot v .
$$

Summing these identities, we obtain $2 \int_{\partial O} \sigma \cdot v=\int_{\Omega} \nabla t \cdot \sigma$. By hypothesis, the value of the latter is $2 S / \varepsilon^{d-1}$, so (2.7) holds true.

$1 /$ Let us establish (2.2). By hypothesis, this identity is true for $\left(\alpha_{-}, \alpha_{+}\right)=$ $(-1,1)$. Let us first assume $-1<\alpha_{-}<\alpha_{+}<1$ and let $O$ be a smooth bounded open set such that

$$
\left[t \leq \alpha_{+}\right] \subset O \subset[t<1] .
$$

For instance, we may slightly mollify $t$ and invoke Sard theorem to define $O$ as a smooth sublevel set of the smooth approximation of $t$. Now let us introduce the truncated function,

$$
\widetilde{t}(y):= \begin{cases}t(y) & \text { if } \alpha_{-} \leq t(y) \leq \alpha_{+} \\ \alpha_{ \pm} & \text {if } \pm t(y)>\alpha_{ \pm}\end{cases}
$$


As above, (2.8) is valid with $\varphi=\widetilde{t}-\alpha_{-}$and we have,

$$
\int_{\Omega_{\alpha_{-}}^{\alpha_{+}}} \nabla t \cdot \sigma=\int_{\Omega_{\alpha_{-}}^{\alpha_{+}}} \nabla \widetilde{t} \cdot \sigma=\left(\alpha_{+}-\alpha_{-}\right) \int_{\partial O} \sigma \cdot v \stackrel{(2.7)}{=}\left(\alpha_{+}-\alpha_{-}\right) S / \varepsilon^{d-1} .
$$

Hence, identity (2.2) holds in the case $-1<\alpha_{-}<\alpha_{+}<1$. The remaining cases follow by continuity of the integral.

$2 /$ We are ready to establish (2.3). Let $-1 \leq \alpha_{-}<\alpha_{+} \leq 1$. By (2.2), the left hand side of (2.3) is bounded by $\int_{\Omega_{\alpha_{-}}^{\alpha_{+}}}|1-\nabla t \cdot \sigma|$.

To estimate this integral, we write

$$
\begin{aligned}
& |1-\nabla t \cdot \sigma|=\left|\left(1-|\nabla t|^{2}\right)+\left(1-|\sigma|^{2}\right)+\right| \nabla t-\left.\sigma\right|^{2} \mid / 2 \\
& \quad \leq|1-| \nabla t||+|1-| \sigma||+\left[(1-|\nabla t|)^{2}+(1-|\sigma|)^{2}+|\nabla t-\sigma|^{2}\right] / 2 .
\end{aligned}
$$

Integrating on $\Omega_{\alpha_{-}}^{\alpha_{+}}$and using the Cauchy-Schwarz inequality, we obtain,

$$
\left|X^{2}-\left(\alpha^{+}-\alpha^{-}\right) S / \varepsilon^{d-1}\right| \leq \sqrt{2 F_{0}} X+F_{0} / 2,
$$

with the notation:

$$
F_{0}:=\mathscr{F}_{0}\left(\sigma, \nabla t, \Omega_{\alpha_{-}}^{\alpha_{+}}\right), \quad X:=\sqrt{\mathscr{H}^{d}\left(\Omega_{\alpha_{-}}^{\alpha_{+}}\right)} .
$$

In particular we have $X^{2}-\sqrt{2 F_{0}} X \leq 2 S / \varepsilon^{d-1}+F_{0} / 2$ which yields $X \leq\left(\sqrt{2 F_{0}}+\right.$ $\left.\sqrt{8 S / \varepsilon^{d-1}+2 F_{0}}\right) / 2$. Substituting this in the right hand side of the above estimate, we obtain,

$\left|X^{2}-\frac{\left(\alpha^{+}-\alpha^{-}\right) S}{\varepsilon^{d-1}}\right| \leq(3 / 2) F_{0}+\sqrt{F_{0}^{2}+4 F_{0} S / \varepsilon^{d-1}} \leq(5 / 2) F_{0}+2 \sqrt{F_{0} S / \varepsilon^{d-1}}$,

that is (2.3).

Similarly, the left hand side of (2.4) is bounded by $\int_{\Omega_{\alpha_{-}}^{\alpha_{+}}}|| \nabla t|-\nabla t \cdot \sigma|$.

Writing ||$\nabla t|-\nabla t \cdot \sigma| \leq|1-\nabla t \cdot \sigma|+|| \nabla t|-1|$, we get $\int_{\Omega_{\alpha_{-}}^{\alpha_{+}}}|| \nabla t|-\nabla t \cdot \sigma| \leq$ $\sqrt{5 F_{0}} X+F_{0} / 2$ which yields (2.4).

\subsection{Construction of a harmonic extension of $t_{\varepsilon}$. Definition of $\Sigma^{s}$}

Recall that $\omega$ is the modulus of continuity of Hypothesis 1 . Let $\delta>0$ be the largest number such that

$$
\delta \leq 1 / 4, \quad \omega(\delta) \leq 1 / 10 .
$$

Then we define

$$
\Omega_{(\varepsilon)}^{\prime}:=\left\{y \in \mathbf{R}^{d}:\left|t_{(\varepsilon)}\right|(y)<9 / 10\right\}, \quad F_{(\varepsilon)}^{\prime}:=\mathbf{R}^{d} \backslash \Omega_{(\varepsilon)}^{\prime},
$$


and

$O_{(\varepsilon), \delta}:=\left\{y \in \mathbf{R}^{d}: d\left(y, F_{(\varepsilon)}^{\prime}\right)>\delta\right\}, \quad F_{(\varepsilon), \delta}:=\overline{\bigcup_{y \in F_{(\varepsilon)}^{\prime}} B_{\delta}(y)}=\mathbf{R}^{d} \backslash O_{(\varepsilon), \delta}$.

Notice that $[|t|<4 / 5] \subset O_{(\varepsilon), \delta}$ or equivalently, $t \geq 4 / 5$ in $F_{(\varepsilon), \delta}$. More precisely, for $y \in F_{(\varepsilon)}^{\prime}$, we have $t \geq 4 / 5$ on $\overline{B_{\delta}}(y)$ if $t(y) \geq 9 / 10$ and $t \leq-4 / 5$ on $\overline{B_{\delta}}(y)$ if $t(y) \leq-9 / 10$.

We introduce the harmonic extension $u_{(\varepsilon)}$ of $t_{(\varepsilon)}$ in $O_{(\varepsilon), \delta}$. Its level sets are good candidates for the hypersurface $\Sigma_{(\varepsilon)}=(1 / \varepsilon) \Sigma_{\varepsilon}$ of Proposition 2.1.b.

Definition 2.1 We set $u_{\varepsilon}(y)=\varepsilon u_{(\varepsilon)}(y / \varepsilon)$, where $u_{(\varepsilon)}$ is defined as

$$
u_{(\varepsilon)}:=\operatorname{argmin}\left\{\int_{\mathbf{R}^{d}}|\nabla \varphi|^{2}: \varphi \in W_{l o c}^{1,2}\left(\mathbf{R}^{d}\right), \varphi \equiv t_{(\varepsilon)} \text { a.e on } F_{(\varepsilon), \delta}\right\} .
$$

Remark 2.1

a) The definition of $u_{(\varepsilon)}$ is valid since the feasible domain of the minimization problem contains at least $t_{(\varepsilon)}$.

b) We do not define $u_{(\varepsilon)}$ as the harmonic extension of $t_{(\varepsilon)}$ in $\Omega_{(\varepsilon)}$ for two reasons. - First, in the sequel, we need $u_{\varepsilon}$ to be equal to $t_{\varepsilon}$ in a large part of $\Omega_{\varepsilon}$. Thanks to the definition of $\Omega_{(\varepsilon)}^{\prime}$, this property holds true in the set $\left[9 / 10<\left|t_{(\varepsilon)}\right|<1\right]$. When we will apply the rigidity estimates of [11] to $u_{(\varepsilon)}$ and $t_{(\varepsilon)}$ in domains intersecting $\left[9 / 10<\left|t_{(\varepsilon)}\right|<1\right]$, this will allow us to use the same averaged normal direction for both vector fields $\nabla u_{\varepsilon}, \nabla t_{\varepsilon}$.

- We also need $u_{(\varepsilon)}$ to be uniformly equicontinuous (independently of $\varepsilon$ ). For this, we define the harmonic extensions $u_{(\varepsilon)}$ in domains satisfying uniformly the exterior ball property. This is the reason for the introduction of the sets $O_{(\varepsilon), \delta}$ which have the exterior ball property with radius $\delta$.

\section{Definition 2.2}

a) For $-1 / 2<s<1 / 2$, we set $\Sigma_{\varepsilon}^{\varepsilon s}:=\varepsilon \Sigma_{(\varepsilon)}^{s}$ where $\Sigma_{(\varepsilon)}^{s}$ is the level set

$$
\Sigma_{(\varepsilon)}^{s}:=\left\{x \in O_{(\varepsilon), \delta}: u_{(\varepsilon)}(x)=s\right\} .
$$

b) For every $z \in O_{(\varepsilon), \delta}$, we set

$$
n_{(\varepsilon)}(z):=\left\{\begin{array}{cl}
\frac{\nabla u_{(\varepsilon)}}{\left|\nabla u_{(\varepsilon)}\right|}(z) & \text { if } \nabla u_{(\varepsilon)}(z) \neq 0, \\
e_{d} & \text { if } \nabla u_{(\varepsilon)}(z)=0 .
\end{array}\right.
$$

Remark 2.2

Since $u_{(\varepsilon)}$ is harmonic in the neighborhood of $\Sigma_{(\varepsilon)}^{s}$, this set is an analytic surface with unit normal $n_{(\varepsilon)}$ in the neighborhood of $\left\{x \in \Sigma_{(\varepsilon)}^{s}: \nabla u_{(\varepsilon)}(x) \neq 0\right\}$.

In the sequel, we drop again the subscripts $(\varepsilon)$ : we note $u$ for $u_{(\varepsilon)}, O_{\delta}$ for $O_{(\varepsilon), \delta}$, $n$ for $n_{(\varepsilon)}$ and $\Sigma^{s}$ for $\Sigma_{(\varepsilon)}^{s}$, etc. We first establish that $\nabla u$ is close to $\nabla t$. 
Lemma 2.2 We have

$$
\int_{\mathbf{R}^{d}}|\nabla u-\sigma|^{2} \leq E_{0} \varepsilon^{3-d} \quad \text { and } \quad \int_{\mathbf{R}^{d}}|\nabla u-\nabla t|^{2} \leq 4 E_{0} \varepsilon^{3-d} .
$$

Proof Since $\sigma$ is divergence free in $O_{\delta}$, we easily check that $u$ minimizes the functional

$$
J(\varphi):=\int_{\mathbf{R}^{d}}|\nabla \varphi-\sigma|^{2} .
$$

in $\left\{\varphi \in W_{\text {loc }}^{1,2}\left(\mathbf{R}^{d}\right): \varphi \equiv t\right.$ in $\left.F_{\delta}\right\}$. Using $\varphi=t$, the energy bound (2.1) yields,

$$
\int_{\mathbf{R}^{d}}|\nabla u-\sigma|^{2}=J(u) \leq J(t) \leq E_{0} .
$$

The second estimates then follows from (2.1) and the triangular inequality.

Let us notice that, by construction, $O_{\delta} \subset B_{R}$ satisfies the exterior ball property with radius $\delta$. As a consequence, we can use the Perron method to obtain the existence of a function $\widetilde{u} \in C\left(\overline{O_{\delta}}\right)$ which is harmonic in $O_{\delta}$ and satisfies $\widetilde{u} \equiv t$ on $\partial O_{\delta}-$ see e.g. [7] Theorem 2.14. By the maximum principle, we have $\widetilde{u}=u$, hence $u$ is continuous on $\overline{O_{\delta}}$. Thereafter we also need $u$ to be uniformly continuous with a modulus of continuity that does not depend on $\varepsilon$. This is the content of the next lemma. We did not find the relevant precise statements in the literature. For the sake of completeness, we provide a proof in Appendix A.

Lemma 2.3 There exists a modulus of continuity $\omega_{\not{q}}^{\star}$ only depending on $\omega$ and $d$ such that $u$ is $\omega_{\dot{\alpha}}^{\star}$-continuous on $\mathbf{R}^{d}$.

Recall that, in $F_{\delta}$, we have $|t| \geq 4 / 5$, so as a consequence of the lemma, the level sets $\left\{\Sigma^{s}\right\}_{-1 / 2<s<1 / 2}$ lie at a positive distance from $F_{\delta}$.

Corollary 2.1 For $-1 / 2<s<1 / 2$, we have

$$
d\left(\Sigma^{s}, F_{\delta}\right) \geq \delta_{\dot{q}}^{\star}, \quad \text { with } \delta_{\dot{q}}^{\star}:=\max \left\{r: \omega_{\dot{q}}^{\star}(r) \leq 2 / 5\right\} .
$$

In particular, $\delta_{\mathscr{q}}^{\star}$ only depends on $\omega$ and $d$.

\subsection{Good cylinders. Bad balls}

We use here the weak rigidity inequalities of [11] (Theorems I.2.2 and I.1.1) to show that $t_{\varepsilon}$ and $u_{\varepsilon}$ are close to some affine function in the neighborhood of points with small local energy. These results are gathered in Lemmas 2.4, 2.5 and 2.6 below whose proof is postponed to Appendix B. They allow us to select the good points where it is possible to carry out the computations and to derive the main estimates leading to the compactness (and lower bound) results. Then, in Lemma 2.7, we bound the volume of "bad" regions. 
Definition 2.3 The local energy in an open set $O \subset \mathbf{R}^{d}$ is defined as

$$
\mathscr{E}(O):=\int_{O \cap \Omega} f_{0}(\sigma, \nabla t)+|\nabla u-\nabla t|^{2}
$$

When $O$ is the open ball $B_{\lambda}(y) \subset \mathbf{R}^{d}$, we use the short hand,

$$
\mathscr{E}_{\lambda}(y):=\mathscr{E}\left(B_{\lambda}(y)\right)=\int_{\Omega \cap B_{\lambda}(y)} f_{0}(\sigma, \nabla t)+|\nabla u-\nabla t|^{2} .
$$

We first show that if $z \in \Omega$ is such that $\mathscr{E}_{3}(z)$ is small enough, then $\Omega-z$ contains a cylinder of the following form.

Definition 2.4 For $\lambda>0$ and $\bar{n} \in S^{d-1}, D_{\lambda}^{\prime}(\bar{n})$ denotes the $(d-1)$-ball,

$$
\left\{y^{\prime} \in \mathbf{R}^{d}:\left|y^{\prime}\right|<\lambda, y^{\prime} \cdot \bar{n}=0\right\}=B_{\lambda} \cap \bar{n}^{\perp} .
$$

For $\lambda>1, \xi \in[0,1)$ and $\bar{n} \in S^{d-1}, D_{\lambda}^{\xi}(\bar{n})$ denotes the finite cylinder

$$
\left\{y^{\prime}+s \bar{n}: y^{\prime} \in D_{\lambda}^{\prime}(\bar{n}),|s|<1-\xi\right\} .
$$

Using rotation invariance, we often consider the case $\bar{n}=e_{d}$, for which we simply write $D_{\lambda}^{\prime}$ for $D_{\lambda}^{\prime}\left(e_{d}\right)$ and $D_{\lambda}^{\xi}$ for

$$
D_{\lambda}^{\xi}\left(e_{d}\right)=\left\{y \in \mathbf{R}^{d}:\left|y_{d}\right|<1-\xi, \sum_{i<d} y_{i}^{2}<\lambda^{2}\right\} .
$$

Lemma 2.4 Let $\xi \in(0,1 / 2)$, and $\eta>0$. There exists $\beta=\beta_{\phi, 1}^{\star}(\xi, \eta)$ such that if $z \in \mathbf{R}^{d}$ satisfies $|u(z)| \leq 1 / 2$ and $\mathscr{E}_{3}(z) \leq \beta$, then, $|\nabla u(z)| \geq 1 / 2$ and using the notation

$$
z_{0}:=z-u(z) n(z), \quad D:=z_{0}+D_{1}^{\xi}(n(z)), \quad \varphi(y)=u(z)+(y-z) \cdot n(z),
$$

we have,

(a) $\bar{D} \subset \Omega$ (see Figure 2.1) and

$$
\|t-\varphi\|_{L^{\infty}(\bar{D})}+\|u-\varphi\|_{L^{\infty}(\bar{D})}+\|\nabla t-\nabla \varphi\|_{L^{2}(D)}+\|\sigma-\nabla \varphi\|_{L^{2}(D)} \leq \eta ;
$$

(b) there exists an analytic mapping $\Psi: D_{1}^{\prime}(n(z)) \times(-1 / 2,1 / 2) \rightarrow \mathbf{R}$ such that $\|\Psi\|_{C^{2}} \leq \eta$ and for every $s \in(-1 / 2,1 / 2)$,

$$
\Sigma^{s} \cap D=\left[z_{0}+\operatorname{sn}(z)\right]+\left\{y^{\prime}+\Psi\left(y^{\prime}, s\right) n(z) ; y^{\prime} \in D_{1}^{\prime}(n(z))\right\} .
$$

(c) As a consequence,

$$
0 \leq \mathscr{H}^{d-1}\left(\Sigma^{s} \cap D\right)-\mathscr{H}^{d-1}\left(D_{1}^{\prime}\right) \leq c_{\not{\phi}}^{\star}(\eta),
$$

with $c_{\mathscr{\varepsilon}}^{\star}(\eta) \downarrow 0$ as $\eta \downarrow 0$. 


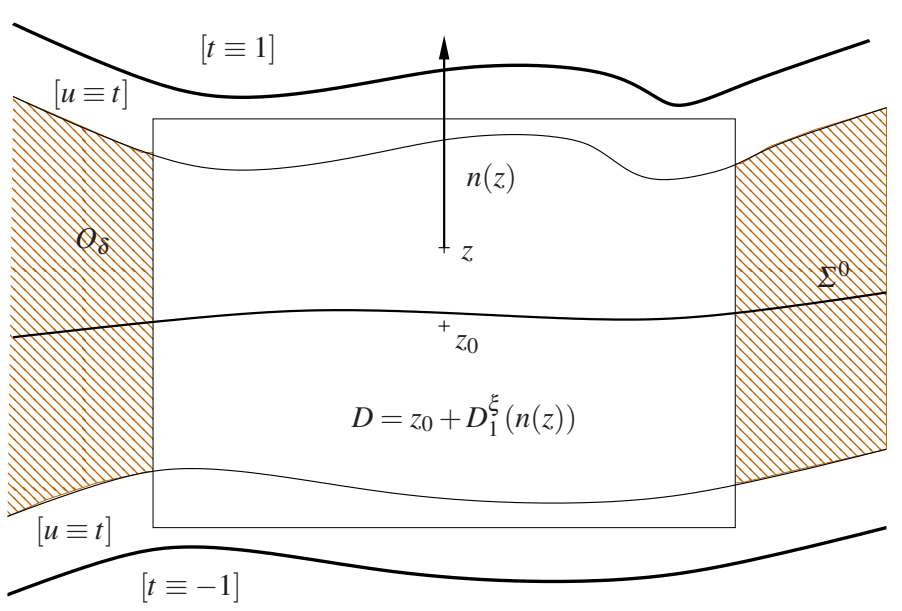

Fig. 2.1 Example of "good" cylinder in dimension $d=2$.

When establishing the lower bound of Theorem 1.2 in Section 4, we apply the weak and strong rigidity inequalities in the cylindrical boxes of Lemma 2.4. It is convenient to gather the relevant estimates here.

Lemma 2.5 Let $\xi \in(0,1 / 4)$. There exists $\beta=\beta_{\dot{\alpha}, 2}^{\star}(\xi)$ such that if $x \in \Sigma^{0}$ satisfies $\mathscr{E}_{3}(x) \leq \beta$, then using the notation $n(x)=\bar{n}$ and

$$
D_{\text {int }}:=x+D_{1-2 \xi}^{2 \xi}(\bar{n}) \quad \subset \quad D:=x+D_{1}^{\xi}(\bar{n}),
$$

we have:

(a) $\bar{D} \subset \Omega$;

(b)

$$
|I I|^{4}(x)+|h|^{2}(x) \leq C \mathscr{E}(D) .
$$

(c) There exists a harmonic function $\psi: D_{\text {int }} \rightarrow \mathbf{R}$ satisfying $\nabla \psi(x)=0, \bar{n} \cdot \nabla \psi \equiv 0$,

$$
\int_{D_{\text {int }}}|\nabla \psi|^{2} \leq C(\xi) \sqrt{\mathscr{E}(D)}
$$

and such that

$$
\int_{D_{\text {int }}}|\nabla u-\bar{n}-\nabla \psi|^{2}+|\nabla t-\bar{n}-\nabla \psi|^{2}+|\sigma-\bar{n}-\nabla \psi|^{2} \leq C(\xi) \mathscr{E}(D) .
$$

In the sequel, we perform some changes in the order of integration for which we are led to consider the sets

$$
\Gamma^{\xi}(z):=\left\{x \in \Sigma^{0}: z \in x+D_{1}^{\xi}(n(x))\right\} .
$$

The purpose of next lemma is to estimate the $(d-1)$-volumes of these sets. 
Lemma 2.6 Let $\xi \in(0,1 / 2)$ and $\eta>0$. There exists $\beta=\beta_{\dot{\varepsilon}, 3}^{\star}(\xi, \eta)>0$ such that if $z \in \mathbf{R}^{d}$ satisfies

$$
|t(z)| \leq 1-\xi \quad \text { and } \quad \mathscr{E}_{3}(z) \leq \beta,
$$

then the function $x \in \Sigma^{0} \mapsto|x-z|^{2}$ admits a unique minimizer $x \in \Sigma^{0}$ and we have $z=x+\operatorname{sn}(x)$ with $|s-t(z)|<\eta$. Moreover, using the notation of Lemma 2.4,

$$
\Gamma^{\xi}(z)=\{x+y+\Psi(y, 0) n(x): y \in X\},
$$

for some open subset $X$ of $n(x)^{\perp}$ such that

$$
D_{1-\eta}^{\prime}(n(x)) \subset X \subset D_{1+\eta}^{\prime}(n(x)) .
$$

In particular,

$$
\left|\mathscr{H}^{d-1}\left(\Gamma^{\xi}(z)\right)-\mathscr{H}^{d-1}\left(D_{1}^{\prime}\right)\right| \leq C \eta \text {. }
$$

We now bound the total volume of points which do not satisfy the assumption $\mathscr{E}_{3}(x)<\beta$. Let us fix $\beta>0$ and let us define the sets of good and bad points as

$$
\mathscr{G}_{\beta}:=\left\{x \in \mathbf{R}^{d}: \mathscr{E}_{\mathcal{B}}(x) \leq \beta\right\}, \quad \mathscr{B}_{\beta}:=\mathbf{R}^{d} \backslash \mathscr{G}_{\beta} .
$$

Lemma 2.7 There exists a finite number of disjoint balls $B_{3}\left(y_{1}\right), \cdots, B_{3}\left(y_{N}\right) \subset \mathbf{R}^{d}$ such that

$$
\mathscr{B}_{\beta} \subset U_{\beta}:=\bigcup_{i=1}^{N} B_{9}\left(y_{i}\right), \quad \text { with } \quad N \leq C_{\not{q}} \varepsilon^{3-d} / \beta .
$$

Proof Since the total energy $\mathscr{E}_{+\infty}(0)$ is bounded by $C E_{0} \varepsilon^{3-d}$, the lemma classically follows from Vitali covering theorem.

As a consequence of the bound on $N$ we have $\mathscr{H}^{d}\left(U_{\beta}\right) \leq C_{\mathscr{q}}(\beta) \varepsilon^{3-d}$ and more generally, for $\beta>0, \lambda \geq 1$,

$$
\mathscr{H}^{d}\left(\cup_{i=1}^{N} B_{9 \lambda}\left(y_{i}\right)\right) \leq C_{\not{\not}}(\beta, \lambda) \varepsilon^{3-d} .
$$

In the sequel, we use this inequality without further reference.

2.4 Definition and properties of the hypersurface $\Sigma_{\varepsilon}$. Proof of Proposition 2.1.b

Before beginning, let us sketch our construction of the hypersurface $\Sigma_{(\varepsilon)}$.

- Step 0 . We introduce a bad set $U_{(\varepsilon)}^{b}$ of points with local energy larger than a fixed value $\beta>0$. We also define the larger open set $U_{(\varepsilon)}^{\star}:=U_{(\varepsilon)}^{b}+B_{9}$ and the good set $G_{(\varepsilon)}^{\star}:=\mathbf{R}^{d} \backslash U_{(\varepsilon)}^{\star}$.

- Step 1. Then, we define $\Sigma_{(\varepsilon)}$ in $G_{(\varepsilon)}^{\star}$ as $\Sigma_{(\varepsilon)}^{\star}:=G_{(\varepsilon)}^{\star} \cap \Sigma_{(\varepsilon)}^{0}=G_{(\varepsilon)}^{\star} \cap\left[u_{(\varepsilon)} \equiv 0\right]$.

- Step 2. Next, we use Corollary I.2.1 to find a level set $\Sigma_{(\varepsilon)}^{s^{b}}$ such that $\Sigma_{(\varepsilon)}^{s^{b}} \cap U_{(\varepsilon)}^{b}$ 
satisfies convenient bounds. We then set $\Sigma_{(\varepsilon)}^{b}:=\Sigma_{(\varepsilon)}^{s^{j}} \cap U_{(\varepsilon)}^{b}$.

- Step 3. Eventually, we complete the construction of $\Sigma_{(\varepsilon)}^{b} \cup \Sigma_{(\varepsilon)}^{\star}$ by adding a smooth hypersurface in the gap $U_{(\varepsilon)}^{\star} \backslash U_{(\varepsilon)}^{b}$ with boundary $\partial \Sigma_{(\varepsilon)}^{\star} \cup \partial \Sigma_{(\varepsilon)}^{b} \subset \partial U_{(\varepsilon)}^{\star} \cup$ $\partial U_{(\varepsilon)}^{b}$.

In Steps 1-3, we also control the volume and the Willmore energy of the respective pieces of hypersurface.

Step 0. Let us fix $\xi=\eta=1 / 4$. With the notation of Corollary I.3.1, Lemma 2.4 and Lemma 2.7, we define,

$$
\beta:=\min \left(\beta_{\dot{\phi}, 1}^{\star}(\xi, \eta), \beta_{\dot{\xi}, 2}^{\star}(\xi)\right), \quad U^{b}:=U_{\beta}=\bigcup_{i=1}^{N} B_{9}\left(y_{i}\right) .
$$

Let us recall that $N \leq C_{\not q} \varepsilon^{3-d}$. We also note

$$
U^{\star}:=\bigcup_{i=1}^{N} B_{18}\left(y_{i}\right), \quad G^{\star}:=\mathbf{R}^{d} \backslash U^{\star} .
$$

Step 1. We set,

$$
\Sigma^{\star}:=\Sigma^{0} \cap G^{\star} .
$$

By Lemma 2.4, $\Sigma^{\star}$ is an analytic hypersurface. Moreover, $\Sigma^{\star} \subset B_{R / \varepsilon}$ and its boundary lies on the spheres $\partial B_{18}\left(y_{i}\right)$.

Now, for every $x \in \Sigma^{\star}$, we have by Corollary 2.1, $B_{\delta_{\dot{\psi}}^{\star}}(x) \subset O_{\delta}$ and applying Corollary I.3.1 with $\varphi=u$ in the ball $B_{\delta_{\xi}^{\star}}(x)$, we obtain

$$
|h|^{2}(x) \leq \frac{C}{\delta_{\dot{q}}^{\star(2+d)}} \int_{B_{\delta_{\xi}^{\star}}(x)}(|\nabla u|-1)^{2},
$$

where $h$ denotes the mean curvature on $\Sigma^{\star}$. Integrating on $\Sigma^{\star}$, and using Fubini, we get

$$
\int_{\Sigma^{\star}}|h|^{2} d \mathscr{H}^{d-1} \leq \frac{C}{\delta_{\mathscr{q}}^{\star(2+d)}} \int_{G^{\star}+B_{\delta_{\mathscr{\xi}}^{\star}}} \mathscr{H}^{d-1}\left(B_{\delta_{\tilde{\xi}}^{\star}}(y) \cap \Sigma^{0}\right)(|\nabla u|(y)-1)^{2} d y .
$$

By Lemma 2.4.b, we have

$$
\mathscr{H}^{d-1}\left(B_{\delta_{\mathscr{q}}^{\star}}(y) \cap \Sigma^{0}\right) \leq C \text { for every } y \text { such that } d\left(y, \Sigma^{\star}\right)<\delta_{\dot{q}}^{\star} .
$$

Hence,

$$
\int_{\Sigma^{\star}}|h|^{2} d \mathscr{H}^{d-1} \leq \frac{C}{\delta_{\dot{\phi}}^{\star(2+d)}} \int_{\mathbf{R}^{d}}(|\nabla u|(y)-1)^{2} d y \leq C_{\not} \varepsilon^{3-d} .
$$

Similarly, using Lemma 2.4.b and Lemma 2.1, we obtain,

$$
\mathscr{H}^{d-1}\left(\Sigma^{\star}\right) \leq C \mathscr{H}^{d}\left(\Omega_{(\varepsilon)}\right) \leq C S \varepsilon^{1-d}+C_{\not \notin} \varepsilon^{2-d} .
$$


Step 2. We now define the hypersurface $\Sigma^{\star}$ inside the balls $B_{18}\left(y_{i}\right)$. Let us first consider the union of interior balls $U^{b}=\cup B_{9}\left(y_{i}\right) \subset \subset U^{\star}$. By Corollary 2.1, for any point $y \in \mathbf{R}^{d}$ such that $|u(y)|<1 / 2$, we have $B_{\delta_{\dot{\varepsilon}}^{\star}}(y) \subset O_{\delta}$, that is $u$ is harmonic in $B_{\delta_{\tilde{\xi}}^{\star}}(y)$. For such a point $y$, we can apply Corollary I.2.1.b to $u$ in $B_{\delta_{\dot{\xi}}^{\star}}(y)$. We get

$$
\int_{\mathbf{R}} \int_{\Sigma^{s} \cap B_{\delta_{\mathscr{\xi}}^{\star} / 2}(y)}\left|I I_{S}\right|^{2} d \mathscr{H}^{d-1} d s \leq C\left(\delta_{\mathscr{\phi}}^{\star}\right) \int_{B_{\delta_{\dot{q}}^{\star}}(y)}|| \nabla u|-1|,
$$

where $I_{S}$ denotes the second fundamental form on $\Sigma^{s}$. Applying Vitali covering theorem to a cover of $\{y \in U:|u(y)|<1 / 2\}$ with balls of the form $B_{\delta_{\tilde{\xi}}^{\star} / 3}(y)$ and summing the estimates, we deduce,

$$
\int_{-1 / 2}^{1 / 2} \int_{\Sigma^{s} \cap U}\left|I I_{S}\right|^{2} d \mathscr{H}^{d-1} d s \leq C\left(\delta_{\dot{\phi}}^{\star}\right) \int_{U^{\star}}|| \nabla u|-1| .
$$

Next, by Cauchy-Schwarz inequality,

$$
\int_{-1 / 2}^{1 / 2} \int_{\Sigma^{s} \cap U}\left|I I_{S}\right|^{2} d \mathscr{H}^{d-1} d s \leq C\left(\delta_{\dot{q}}^{\star}\right) \sqrt{\mathscr{H}^{d}\left(U^{\star}\right) \int_{\mathbf{R}^{d}}(|\nabla u|-1)^{2}} \leq C_{\not \dot{q}} \varepsilon^{3-d} .
$$

On the other hand, by Corollary I.2.1.a, we have

$$
\int_{\mathbf{R}} \mathscr{H}^{d-1}\left(\Sigma^{s} \cap U\right) d s \leq \mathscr{H}^{d}(U)+C_{\not \notin} \varepsilon^{3-d} \leq C_{\not \subset} \varepsilon^{3-d} .
$$

Let us now introduce a small parameter $s_{0} \in(0,1 / 4)$ to be fixed later. From the above bounds, there exists $s^{b} \in\left[-s_{0}, s_{0}\right]$ such that $\Sigma^{b}:=\Sigma^{s^{b}} \cap U^{b}$ is an analytic hypersurface satisfying

$$
\mathscr{H}^{d-1}\left(\Sigma^{b}\right)+\int_{\Sigma^{b}}\left|I I^{b}\right|^{2} d \mathscr{H}^{d-1} \leq C_{\not \notin} \varepsilon^{3-d} / s_{0} .
$$

Step 3. We have to build a hypersurface in $U^{\star} \backslash U^{b}$ connecting $\Sigma^{\star}$ to $\Sigma^{b}$. For this, let us introduce a nonincreasing cut-off function $\chi \in C^{\infty}\left(\mathbf{R}_{+}\right)$such that $\chi \equiv 1$ on $[0,1], \chi \equiv 0$ on $[3,+\infty)$ and $0 \geq \chi^{\prime} \geq-1$. We also introduce a nondecreasing truncating function $T \in C^{\infty}\left(\mathbf{R}_{+}\right)$such that $T(0)=0, T \equiv 1$ on $[1,+\infty)$ and $0 \leq$ $T^{\prime} \leq 2$. We then set

$\Sigma:=\left\{x \in \mathbf{R}^{d}: u(x)-s^{b} \boldsymbol{\theta}(x)=0\right\} \quad$ with $\quad \theta(y):=T\left(\sum_{i=1}^{N} \chi\left(d\left(y, B_{9}\left(y_{i}\right)\right)\right)\right.$.

Since $\theta \equiv 1$ in $U^{b}$ and $\theta \equiv 0$ in $G^{\star}$, we obviously have $\Sigma \cap G^{\star}=\Sigma^{\star}, \Sigma \cap U^{b}=\Sigma^{b}$ and in fact $\Sigma=\Sigma^{s^{b}}$ in some neighborhood of $\overline{U^{b}}, \Sigma=\Sigma^{0}$ in some neighborhood of $G^{\star}$. Notice that the balls $B_{3}\left(y_{i}\right)$ are disjoint. Hence, there exists $K \geq 0$, only depending on $d$ such that the number $P(y)$ of non-zero elements in the sum which defines $\theta(y)$ is bounded by $K$. 
Let us note $w(y)=u(y)-s^{b} \theta$ and let us fix $z \in \Sigma \cap\left[U^{\star} \backslash U\right]$. We compute at point $z$,

$$
|\nabla w|(z) \geq|\nabla u|(z)-\left|s^{b}\right||\nabla \theta|(z) \geq 1 / 2-2\left|s^{b}\right| P(z)\left\|\chi^{\prime}\right\|_{\infty} \geq 1 / 2-2 K s_{0} .
$$

Now, we fix

$$
s_{0}:=1 /(8 K)
$$

so that

$$
|\nabla w|(z) \geq 1 / 4
$$

Consequently, $\Sigma=w^{-1}(\{0\})$ is a smooth hypersurface.

Let us now estimate the $(d-1)$-volume and the $L^{2}$ norm of the second fundamental form of $\Sigma \cap\left[U^{\star} \backslash U\right]$. We consider again a point $z \in \Sigma \cap\left[U^{\star} \backslash U\right]$. We have $|u(z)| \leq\left|s^{b}\right| \leq 1 / 4$ and by construction, $\mathscr{E}_{3}(z) \leq \beta_{\mathscr{\varepsilon}, 1}^{\star}(1 / 4,1 / 4)$ so we can apply Lemma 2.4 at this point with $\xi=\eta=1 / 4$. Assuming without loss of generality that $z=0, n(z)=e_{d}$ and using the notation of Lemma 2.4.b we have for $y^{\prime} \in D_{1}^{\prime}$ and $-1 / 2<s<1 / 2$,

$$
w\left(y^{\prime}+s e_{d}\right)=0 \quad \Longleftrightarrow \Psi\left(y^{\prime}, s^{b} \theta\left(y^{\prime}+s e_{d}\right)\right)=s
$$

Now, let us set,

$$
Z\left(y^{\prime}, s\right):=s-\Psi\left(y^{\prime}, s_{\star} \theta\left(y^{\prime}+s e_{d}\right)\right), \quad \text { for } y^{\prime} \in D_{1}^{\prime},-1 / 2 \leq s \leq 1 / 2 .
$$

We have $\pm Z\left(y^{\prime}, \pm 1 / 2\right) \geq 1 / 2-\eta=1 / 4>0$ and $\frac{d}{d s} Z\left(y^{\prime}, s\right) \geq 1-\eta\left|s_{0}\right|\left\|\theta^{\prime}\right\|_{\infty} \geq$ $15 / 16>0$, so for every $y^{\prime} \in D_{1}^{\prime}$, the equation $Z\left(y^{\prime}, s\right)=0$ admits a unique solution $s=\zeta\left(y^{\prime}\right)$. In other words, $\left[D_{1}^{\prime} \times(-1 / 2,1 / 2) e_{d}\right] \cap \Sigma$ is the graph of the mapping $\zeta$. By regularity of $\Psi$ and $\theta$, we also have

$$
\|\nabla \zeta\|_{\infty} \leq C, \quad\left\|D^{2} \zeta\right\|_{\infty} \leq C .
$$

We deduce the inequality

$$
\mathscr{H}^{d-1}\left(\left[D_{1}^{\prime} \times(-1 / 2,1 / 2) e_{d}\right] \cap \Sigma\right)+\int_{\left[D_{1}^{\prime} \times(-1 / 2,1 / 2) e_{d}\right] \cap \Sigma}|I I|^{2} d \mathscr{H}^{d-1} \leq C .
$$

Using Vitali covering theorem and the bound $\mathscr{H}^{d}\left(U^{\star}\right) \leq C_{\not{g}} \varepsilon^{3-d}$, we get,

$$
\mathscr{H}^{d-1}\left(\Sigma \cap U^{\star}\right)+\int_{\Sigma \cap U^{\star}}|I I|^{2} d \mathscr{H}^{d-1} \leq C_{\not d} \varepsilon^{3-d} .
$$

Taking into account (2.16),(2.17),(2.18) and (2.19), we have established

$$
\mathscr{H}^{d-1}(\Sigma) \leq C S \varepsilon^{1-d}+C_{\not}, \quad \text { and } \quad \mathscr{W}(\Sigma) \leq C_{\not \notin} \varepsilon^{3-d}
$$

Eventually, we set $M:=\left\{y \in \mathbf{R}^{d}: t(y)=-1\right\}$ and $O:=\left\{y \in \mathbf{R}^{d}: w(y)=\right.$ $\left.u(y)-s_{\star} \theta(y)<0\right\}$. We have $O \subset B_{R / \varepsilon}$ and $\partial O=\Sigma$. Moreover, taking into account Lemma 2.1 and the bound $\mathscr{H}^{d}\left(U^{\star}\right) \leq C_{\not \notin} \varepsilon^{3-d}$, we have

$$
\left\|\mathbf{1}_{O}-\mathbf{1}_{M}\right\|_{L^{1}} \leq 2 S \varepsilon^{1-d}+C_{\not} \varepsilon^{2-d} .
$$


Putting back the subscripts $(\varepsilon)$, we note $\Sigma_{(\varepsilon)}:=\Sigma, O_{(\varepsilon)}:=O, M_{(\varepsilon)}:=M, U_{(\varepsilon)}^{\star}:=$ $U^{\star}$ and $G_{(\varepsilon)}^{\star}:=G^{\star}$. Returning to the original variables, we set $\Sigma_{\varepsilon}:=(1 / \varepsilon) \Sigma_{(\varepsilon)}$, $O_{\varepsilon}:=(1 / \varepsilon) O_{(\varepsilon)}$ and $M_{\varepsilon}:=(1 / \varepsilon) M_{(\varepsilon)}$. Unscaling the inequalities (2.20)(2.21), we obtain the estimates of Proposition 2.1.b.

Further conclusions. For later use, we remark here that

$$
\mathscr{H}^{d-1}\left(B_{1}(y) \cap \Sigma_{(\varepsilon)}\right) \leq C, \quad \text { for every } y \in G^{\star} .
$$

Indeed, by construction, $B_{1}(y) \cap \Sigma_{(\varepsilon)}=B_{1}(y) \cap \Sigma_{(\varepsilon)}^{0}$ for every $y \in G_{(\varepsilon)}^{\star}$ and we can apply Lemma 2.4.b to any point $z \in B_{1}(y) \cap \Sigma_{(\varepsilon)}$.

In the sequel, we consider bad sets which write, using the notation of Lemma 2.7, as $\mathscr{U}_{(\varepsilon)}=U_{(\varepsilon), \beta^{\prime}}$. We deduce from Lemma 2.7 (2.19) and (2.22) that

$$
\mathscr{H}^{d-1}\left(\Sigma_{(\varepsilon)} \cap\left(U_{(\varepsilon)}^{\star} \cup \mathscr{U}_{(\varepsilon)}\right)\right) \leq C_{\bar{q}}\left(\beta^{\prime}\right) \varepsilon^{3-d}
$$

More generally, for $\lambda>0$, we have

$$
\mathscr{H}^{d-1}\left(\Sigma_{(\varepsilon)} \cap\left[\left(U_{(\varepsilon)}^{\star} \cup \mathscr{U}_{(\varepsilon)}\right)+B_{\lambda}\right]\right) \leq C_{\not}\left(\beta^{\prime}, \lambda\right) \varepsilon^{3-d} .
$$

\subsection{Proof of Proposition 2.1.c}

In the previous section, we have built the open sets $O_{\varepsilon}$ and $\Sigma_{\varepsilon}$ satisfying the estimates of Proposition 2.1.b. We now establish Proposition 2.1.c. Let $\varphi \in C\left(\mathbf{R}^{d} \times\right.$ $\left.\mathbf{R}^{d}\right)$ such that $\sup \left[|\varphi(y, v)| /\left(1+|v|^{2}\right)\right]<\infty$. In scaled variables, we have to prove that

$$
\varepsilon^{d-1}\left|\frac{1}{2} \int_{\Omega_{(\varepsilon)}} \varphi\left(\varepsilon z, \nabla t_{(\varepsilon)}(z)\right) d z-\int_{\Sigma_{(\varepsilon)}} \varphi\left(\varepsilon x, v_{(\varepsilon)}(x)\right) d \mathscr{H}^{d-1}(x)\right| \stackrel{\varepsilon \downarrow 0}{\longrightarrow} 0 .
$$

Proof (of (2.24))

Step 1. Mollification of $\varphi$

Let us introduce the quantities,

$Q_{(\varepsilon)}[\varphi]:=\frac{1}{2} \int_{\Omega_{(\varepsilon)}} \varphi\left(\varepsilon z, \nabla t_{(\varepsilon)}(z)\right) d z, \quad Q_{(\varepsilon)}^{\prime}[\varphi]:=\int_{\Sigma_{(\varepsilon)}} \varphi\left(\varepsilon x, v_{(\varepsilon)}(x)\right) d \mathscr{H}^{d-1}(x)$

Let $\chi \in C_{c}^{\infty}\left(\mathbf{R}^{d},[0,1]\right)$ be a cut-off function satisfying $\chi \equiv 1$ in $B_{2}$ and let us set $\varphi^{\prime}(y, v)=\chi(v) \varphi(y, v)$. We obviously have $Q_{(\varepsilon)}^{\prime}\left[\varphi^{\prime}\right]=Q_{(\varepsilon)}^{\prime}[\varphi]$. On the other hand, we compute

$$
\varepsilon^{d-1}\left|Q_{(\varepsilon)}\left[\varphi^{\prime}-\varphi\right]\right| \leq \frac{\varepsilon^{d-1}}{2} \int_{\left[\left|\nabla t_{\varepsilon}\right|>2\right]}|\varphi|\left(\varepsilon z, \nabla t_{(\varepsilon)}(z)\right) d z .
$$


Since $|\varphi|(y, v) \leq C(\varphi)(|v|-1)^{2}$ for $|v|>2$, this leads to,

$$
\varepsilon^{d-1}\left|Q_{(\varepsilon)}\left[\varphi^{\prime}-\varphi\right]\right| \leq \frac{C(\varphi) \varepsilon^{d-1}}{2} \int_{\Omega_{(\varepsilon)}}\left(\left|\nabla t_{(\varepsilon)}\right|-1\right)^{2} \leq \frac{C(\varphi) E_{0} \varepsilon^{2}}{2} \stackrel{\varepsilon \downarrow 0}{\rightarrow} 0 .
$$

As a consequence, we may assume that $\varphi$ is compactly supported in $B_{R+1} \times B_{4}$.

Let $\widetilde{\varphi} \in C_{c}\left(\mathbf{R}^{d} \times \mathbf{R}^{d}\right)$, we deduce from the estimates of Proposition 2.1.b,

$$
\begin{gathered}
\varepsilon^{d-1}\left|Q_{(\varepsilon)}[\widetilde{\varphi}-\varphi]\right| \leq \varepsilon^{d-1} \mathscr{H}^{d}\left(\Omega_{(\varepsilon)}\right)\|\widetilde{\varphi}-\varphi\|_{\infty} / 2 \leq C_{\mathscr{\xi}}\|\widetilde{\varphi}-\varphi\|_{\infty}, \\
\varepsilon^{d-1}\left|Q_{(\varepsilon)}^{\prime}[\widetilde{\varphi}-\varphi]\right| \leq \varepsilon^{d-1} \mathscr{H}^{d-1}\left(\Sigma_{(\varepsilon)}\right)\|\widetilde{\varphi}-\varphi\|_{\infty} \leq C_{\mathscr{\xi}}\|\widetilde{\varphi}-\varphi\|_{\infty} .
\end{gathered}
$$

Thus, by density, we may also assume that $\varphi$ is smooth and compactly supported.

Step 2. Cut-off procedure.

From now on, we consider a fixed test function $\varphi \in \mathscr{D}\left(\mathbf{R}^{d} \times \mathbf{R}^{d}\right)$. In order to ease the estimate below, we perform partitions of $\mathbf{R}^{d}$ into good sets and bad sets. Let us introduce a small parameter $\xi \in(0,1 / 2)$ and let us set

$$
\Omega_{(\varepsilon)}^{\xi / 2}:=\left\{z \in \Omega_{(\varepsilon)}:\left|t_{(\varepsilon)}\right|(z)<1-\xi / 2\right\} .
$$

By Lemma 2.4 and Lemma 2.5 , there exists $\beta_{a}>0$ only depending on $\xi$ such that if $x \in \Sigma_{(\varepsilon)}^{0}$ satisfies $\mathscr{E}_{(\varepsilon), 3}(x)<\beta_{a}$ then

$$
x+D_{1}^{\xi}\left(n_{(\varepsilon)}(x)\right) \subset \Omega_{(\varepsilon)}^{\xi / 2},
$$

and we have the estimate,

$$
\left.\frac{1}{\mathscr{H}^{d}\left(D_{1}^{0}\right)} \int_{\left[x+D_{1}^{\xi}\left(n_{(\varepsilon)}(x)\right)\right]} \mid \nabla t_{(\varepsilon)}(z)-n_{(\varepsilon)}(x)\right) \mid d z \leq \xi
$$

Now, for $z \in \Omega_{(\varepsilon)}^{\xi / 2}$, we set

$$
q_{(\varepsilon)}^{\xi}(z):=\frac{1}{\mathscr{H}^{d}\left(D_{1}^{0}\right)} \int_{\Sigma_{(\varepsilon)}^{0}} \theta^{\xi}\left(z-x, n_{(\varepsilon)}(x)\right) d \mathscr{H}^{d-1}(x),
$$

where for $\bar{n} \in S^{d-1}, y \mapsto \theta^{\xi}(y, \bar{n})$ denotes the characteristic function of $D_{1}^{\xi}(\bar{n})$.

By Lemma 2.6 there exists $\beta_{b}>0$ only depending on $\xi$ such that if $z \in \Omega_{(\varepsilon)}^{\xi / 2}$ satisfies $\mathscr{E}_{(\varepsilon), 3}(z)<\beta_{b}$, then

$$
\left|q_{(\varepsilon)}^{\xi}(z)-1\right| \leq \xi
$$

Let us set $\beta:=\min \left(\beta_{a}, \beta_{b}\right)$ and let us define the bad sets

$$
\mathscr{U}_{(\varepsilon), \xi}:=\left[U_{(\varepsilon)}^{\star} \cup U_{(\varepsilon), 3, \beta}\right]+B_{3} \quad \subset \quad \mathscr{U}_{(\varepsilon), \xi}^{\prime}:=\mathscr{U}_{(\varepsilon), \xi}+B_{6},
$$


and the corresponding good sets

$$
\mathscr{G}_{(\varepsilon), \xi}:=\mathbf{R}^{d} \backslash \mathscr{U}_{(\varepsilon), \xi} \quad \supset \quad \mathscr{G}_{(\varepsilon), \xi}^{\prime}:=\mathbf{R}^{d} \backslash \mathscr{U}_{(\varepsilon), \xi}^{\prime} .
$$

By Lemma 2.7, we have

$$
\mathscr{H}^{d-1}\left(\mathscr{U}_{(\varepsilon), \xi}^{\prime}\right) \leq C_{\not}(\xi) \varepsilon^{3-d}
$$

We now introduce a cut-off function $\chi_{(\varepsilon), \xi} \in C^{\infty}\left(\mathbf{R}^{d},[0,1]\right)$ such that

$$
\chi_{(\varepsilon), \xi} \equiv 1 \text { on } \mathscr{G}_{(\varepsilon), \xi}^{\prime}, \quad \chi_{(\varepsilon), \xi} \equiv 0 \text { on } \mathscr{U}_{(\varepsilon), \xi}, \quad\left\|\nabla \chi_{(\varepsilon), \xi}\right\|_{\infty} \leq 1
$$

We set $\varphi_{\xi}(y, n):=\chi_{(\varepsilon), \xi}((1 / \varepsilon) y) \varphi(y, n)$. Note the abuse of notation: although not explicitly indicated, $\varphi_{\xi}$ depends on $\varepsilon$. We have,

$$
\begin{aligned}
& \varepsilon^{d-1}\left|Q_{(\varepsilon)}\left[\varphi_{\xi}-\varphi\right]\right| \leq\|\varphi\|_{\infty} \varepsilon^{d-1} \mathscr{H}^{d}\left(\mathscr{U}_{(\varepsilon), \xi}^{\prime}\right) / 2 \stackrel{(2.27)}{\leq} C_{\dot{q}}(\varphi, \xi) \varepsilon^{2} \\
& \varepsilon^{d-1}\left|Q_{(\varepsilon)}^{\prime}\left[\varphi_{\xi}-\varphi\right]\right| \leq\|\varphi\|_{\infty} \varepsilon^{d-1} \mathscr{H}^{d-1}\left(\Sigma_{(\varepsilon)} \cap \mathscr{U}_{(\varepsilon), \xi}^{\prime}\right) \stackrel{(2.23)}{\leq} C_{\not \varepsilon}(\varphi, \xi) \varepsilon^{2} .
\end{aligned}
$$

Therefore,

$$
\varepsilon^{d-1}\left|\left\{Q_{(\varepsilon)}\left[\varphi_{\xi}\right]-Q_{(\varepsilon)}^{\prime}\left[\varphi_{\xi}\right]\right\}-\left\{Q_{(\varepsilon)}[\varphi]-Q_{(\varepsilon)}^{\prime}[\varphi]\right\}\right| \leq C_{\not \notin}(\varphi, \xi) \varepsilon^{2} \stackrel{\varepsilon \downarrow 0}{\longrightarrow} 0 .
$$

As a consequence, we can substitute $\varphi_{\xi}$ for $\varphi$ in (2.24). The rest of the proof consists in establishing

$$
\underset{\varepsilon \downarrow 0}{\limsup \varepsilon^{d-1}}\left|Q_{(\varepsilon)}\left[\varphi_{\xi}\right]-Q_{(\varepsilon)}^{\prime}\left[\varphi_{\xi}\right]\right| \leq C_{\xi}(\varphi) \xi
$$

Since $\xi \in(0,1 / 2)$ is arbitrary, this proves the desired convergence result (2.24).

Step 3. Proof of (2.28).

Let us enumerate the benefits of the cut-off procedure. Since $\varphi_{\xi}$ is supported in $\mathscr{G}_{\varepsilon, \xi} \times \mathbf{R}^{d}$, we may substitute $\Sigma_{(\varepsilon)}^{0}$ for $\Sigma_{(\varepsilon)}$ in the definition of $Q_{(\varepsilon)}^{\prime}\left[\varphi_{\xi}\right]$. Moreover (2.25) hold for any $x \in \Sigma_{(\varepsilon)}^{0} \cap \mathscr{G}_{(\varepsilon), \xi}$ and (2.26) hold for any $z \in \Omega_{(\varepsilon)}^{\xi / 2} \cap \mathscr{G}_{(\varepsilon), \xi}$. Eventually, $\varphi_{\xi}$ satisfies

$$
\begin{gathered}
\left\|\varphi_{\xi}\right\|_{\infty} \leq\|\varphi\|_{\infty}, \quad\left\|\nabla_{n} \varphi_{\xi}\right\|_{\infty} \leq\left\|\nabla_{n} \varphi\right\|_{\infty} \\
\left\|\nabla_{x} \varphi_{\xi}\right\|_{L^{\infty}\left(\mathscr{G}_{\varepsilon, \xi}^{\prime} \times \mathbf{R}^{d}\right)} \leq\left\|\nabla_{x} \varphi\right\|_{\infty}, \quad\left\|\nabla_{x} \varphi_{\xi}\right\|_{\infty} \leq \varepsilon^{-1}\|\varphi\|_{\infty}+\left\|\nabla_{x} \varphi\right\|_{\infty}
\end{gathered}
$$

To lighten notation we write $Q_{(\varepsilon)}$ for $Q_{(\varepsilon)}\left[\varphi_{\varepsilon, \xi}\right]$ and $Q_{(\varepsilon)}^{\prime}$ for $Q_{(\varepsilon)}^{\prime}\left[\varphi_{\varepsilon, \xi}\right]$. With this notation, we have to show that

$$
\underset{\varepsilon \downarrow 0}{\limsup } \varepsilon^{d-1}\left|Q_{(\varepsilon)}-Q_{(\varepsilon)}^{\prime}\right| \leq C_{\not{\xi}}(\varphi) \xi .
$$


For this, we introduce the intermediate quantities

$$
Q_{(\varepsilon)}(\xi):=\frac{1}{2} \int_{\Omega_{(\varepsilon)}^{\xi / 2}} \varphi_{\varepsilon, \xi}\left(\varepsilon z, \nabla t_{(\varepsilon)}(z)\right) d z
$$

and

$$
Q_{(\varepsilon)}^{\prime}(\xi):=\int_{\Sigma_{(\varepsilon)}^{0}}\left\{\frac{1}{\mathscr{H}^{d}\left(D_{1}^{0}\right)} \int_{\left[x+D_{1}^{\xi}\left(n_{(\varepsilon)}(x)\right)\right]} \varphi_{\varepsilon, \xi}\left(\varepsilon z, \nabla t_{(\varepsilon)}(z)\right) d z\right\} d \mathscr{H}^{d-1}(x) .
$$

We prove (2.28) by estimating successively $Q_{(\varepsilon)}-Q_{(\varepsilon)}(\xi), Q_{(\varepsilon)}(\xi)-Q_{(\varepsilon)}^{\prime}(\xi)$ and $Q_{(\varepsilon)}^{\prime}(\xi)-Q_{(\varepsilon)}^{\prime}$. In these estimates, apart from the conclusions, we drop the subscripts $(\varepsilon)$.

Step 3.1. Estimating $Q_{(\varepsilon)}-Q_{(\varepsilon)}(\xi)$.

Using (2.3) in Lemma 2.1 with $\left(\alpha_{-}, \alpha_{+}\right)=(-1,-1+\xi / 2)$ and $\left(\alpha_{-}, \alpha_{+}\right)=$ $(1-\xi / 2,1)$, we obtain,

$$
\begin{aligned}
|Q-Q(\xi)| \leq \frac{\|\varphi\|_{\infty}}{2} \mathscr{H}^{d}\left(\Omega^{-1,-1+\xi / 2} \cup \Omega^{1-\xi / 2,1}\right) & \\
& \leq \frac{\|\varphi\|_{\infty}}{2}\left(\frac{S \xi}{\varepsilon^{d-1}}+\frac{C_{\not{\phi}}(\xi)}{\varepsilon^{(d-1) / 2}}\right) .
\end{aligned}
$$

Hence,

$$
\underset{\varepsilon \downarrow 0}{\limsup } \varepsilon^{d-1}\left|Q_{(\varepsilon)}-Q_{(\varepsilon)}(\xi)\right| \leq\|\varphi\|_{\infty} S \xi / 2 .
$$

Step 3.2. Estimating $Q_{(\varepsilon)}(\xi)-Q_{(\varepsilon)}^{\prime}(\xi)$.

Changing the order of integration in the definition of $Q^{\prime}(\xi)$, we obtain,

$$
Q^{\prime}(\xi)=\int_{\Omega^{\xi} / 2} \varphi_{\varepsilon, \xi}(\varepsilon z, \nabla t(z)) q^{\xi}(z) d z,
$$

Hence, by (2.26), we have,

$$
\left|Q(\xi)-Q^{\prime}(\xi)\right| \leq\|\varphi\|_{\infty} \mathscr{H}^{d}(\Omega) \xi \stackrel{(2.3)}{\leq}\left(\frac{S}{\varepsilon^{d-1}}+\frac{C_{\not \notin}}{\varepsilon^{(d-1) / 2}}\right) \xi .
$$

Again, this leads to

$$
\underset{\varepsilon \downarrow 0}{\limsup } \varepsilon^{d-1}\left|Q_{(\varepsilon)}(\xi)-Q_{(\varepsilon)}^{\prime}(\xi)\right| \leq\|\varphi\|_{\infty} S \xi .
$$

Step 3.3. Estimating $Q^{\prime}(\xi)-Q^{\prime}$.

We have

$$
\left|Q^{\prime}(\xi)-Q^{\prime}\right| \leq \int_{\Sigma^{0}} q(x) d \mathscr{H}^{d-1}(x)
$$


with

$$
q(x):=\left|\varphi_{\xi}(\varepsilon x, n(x))-\frac{1}{\mathscr{H}^{d}\left(D_{1}^{0}\right)} \int_{\left[x+D_{1}^{\xi}(n(x))\right]} \varphi_{\xi}(\varepsilon z, \nabla t(z)) d z\right|
$$

For $x \in \Sigma^{0} \cap \mathscr{G}_{\xi}^{\prime}$, we estimate $q(x)$ as follows.

$$
\begin{gathered}
q(x) \leq\left|\varphi_{\xi}(\varepsilon x, n(x))\right|\left[1-\mathscr{H}^{d}\left(D_{1}^{\xi}\right) / \mathscr{H}^{d}\left(D_{1}^{0}\right)\right] \\
+\frac{1}{\mathscr{H}^{d}\left(D_{1}^{0}\right)} \int_{\left[x+D_{1}^{\xi}(n(x))\right]}\left|\varphi_{\xi}(\varepsilon z, \nabla t(z))-\varphi_{\xi}(\varepsilon x, n(x))\right| d z \\
\quad \leq 2\left\|\varphi_{\xi}\right\|_{\infty} \xi \\
+\left[\sqrt{2}\left\|\nabla_{x} \varphi_{\xi}\right\|_{L^{\infty}\left(x+D_{1}^{\xi}(n(x))\right)} \varepsilon+\frac{\left\|\nabla_{\tau} \varphi_{\xi}\right\|_{\infty}}{\mathscr{H}^{d}\left(D_{1}^{0}\right)} \int_{\left[x+D_{1}^{\xi}(n(x))\right]}|\nabla t(z)-n(x)| d z\right] \\
\stackrel{(2.25)}{\leq}\left(2\left\|\varphi_{\xi}\right\|_{\infty}+\left\|\nabla_{\tau} \varphi_{\xi}\right\|_{\infty}\right) \xi+\sqrt{2}\left\|\nabla_{x} \varphi_{\xi}\right\|_{L^{\infty}\left(x+D_{1}^{\xi}(n(x))\right)} \varepsilon .
\end{gathered}
$$

Integrating over $\Sigma_{0} \cap \mathscr{G}_{\xi}^{\prime}$ and using (2.29), (2.30) we obtain,

$$
\begin{aligned}
& \left|Q^{\prime}(\xi)-Q^{\prime}\right| \\
& \quad \leq 2\|\varphi\|_{W^{1, \infty}} \mathscr{H}^{d-1}(\Sigma)(\xi+\varepsilon)+\sqrt{2}\|\varphi\|_{\infty} \mathscr{H}^{d-1}\left(\Sigma \cap\left[\mathscr{U}_{\xi}^{\prime}+B_{\sqrt{2}}\right]\right) \\
& \stackrel{(2.20)(2.23)}{\leq} C_{\phi}(\varphi)\left(\varepsilon^{d-1} \xi+\varepsilon^{d}\right) .
\end{aligned}
$$

We conclude that

$$
\underset{\varepsilon \downarrow 0}{\limsup } \varepsilon^{d-1}\left|Q_{(\varepsilon)}^{\prime}(\xi)-Q_{(\varepsilon)}^{\prime}\right| \leq C_{\not \notin}(\varphi) \xi .
$$

Step 3.4. Conclusion.

Gathering (2.31)(2.32)(2.33), we have established (2.28). This proves (2.24).

\subsection{Proof of Theorem 1.1}

Let $R, S>0$ and let us consider a family $\left\{a_{\varepsilon}\right\}_{0<\varepsilon<1}$ with $a_{\varepsilon}=\left(\sigma_{\varepsilon}, \nabla t_{\varepsilon}, \Omega_{\varepsilon}\right) \in$ $\mathscr{A}_{\mathcal{E}}(R, S)$ satisfying the hypotheses of Theorem 1.1. In this case, the conclusions of Proposition 2.1 hold. We use the notation of the proposition.

Let us call $\mathscr{V}_{\varepsilon}^{\prime}=\mathscr{V}\left(\Sigma_{\varepsilon}, n_{\varepsilon}\right)$ the oriented $(d-1)$-varifold associated with $\Sigma_{\varepsilon}=$ $\partial O_{\varepsilon}$ and let $\varphi \in C\left(\mathbf{R}^{d} \times S^{d-1}\right)$. Proposition 2.1.c applied to the test function $\bar{\varphi}(y, v)=\varphi(y, v /|v|) \chi^{\star}(|v|)$ reads

$$
\left\langle\mathscr{V}_{\varepsilon}^{\star}\left(a_{\varepsilon}\right) ; \varphi\right\rangle-\left\langle\mathscr{V}_{\varepsilon}^{\prime} ; \bar{\varphi}\right\rangle \rightarrow 0 \text { as } \varepsilon \downarrow 0 .
$$


Now, since $\chi^{\star}(1)=1$, we have $\left\langle\mathscr{V}_{\varepsilon}^{\prime} ; \bar{\varphi}\right\rangle=\left\langle\mathscr{V}_{\varepsilon}^{\prime} ; \varphi\right\rangle$ and we conclude that

$$
\mathscr{V}_{\varepsilon}^{\star}\left(a_{\varepsilon}\right)-\mathscr{V}_{\varepsilon}^{\prime} \rightarrow 0 \quad \text { weakly in } \mathscr{M}\left(\mathbf{R}^{d} \times S^{d-1}\right) \text { as } \varepsilon \downarrow 0 .
$$

As a consequence, the compactness and limit properties of $\left\{\mathscr{V}_{\varepsilon}^{\prime}\right\}$ transfer to the family $\left\{\mathscr{V}_{\varepsilon}^{\star}\left(a_{\varepsilon}\right)\right\}$ and it is sufficient to study the former.

The Radon measures $\left\{\mathscr{V}_{\varepsilon}^{\prime}\right\}$ are non-negative and supported in $B_{R+C} \times S^{d-1}$. By the second estimate of Proposition 2.1.b, their total mass $\left\|\mathscr{V}_{\varepsilon}^{\prime}\right\|\left(\mathbf{R}^{d} \times S^{d-1}\right)=$ $\left\langle\mathscr{V}_{\varepsilon}^{\prime} ; 1\right\rangle$ is uniformly bounded. Thus, there exists a (not relabelled) sequence $\varepsilon \downarrow 0$ and an oriented $(d-1)$-varifold $\mathscr{V}_{0}$ such that up to extraction,

$$
\mathscr{V}_{\varepsilon}^{\prime} \rightarrow \mathscr{V}_{0} \quad \text { in } \mathscr{M}\left(\mathbf{R}^{d} \times S^{d-1}\right) \text { as } \varepsilon \downarrow 0 .
$$

The estimates of Proposition 2.1.b show that the sequence of varifolds $\mathscr{V}_{\varepsilon}^{\prime}=$ $\mathscr{V}\left(\Sigma_{\varepsilon}, v_{\varepsilon}\right)$ satisfies the requirements of (1.3). Therefore, $\mathscr{V}_{0} \in \mathscr{A}_{0}(R, S)$. This establishes parts (c) of the theorem.

We easily see that $\mathscr{V}_{0}$ is non-negative and compactly supported in $\overline{B_{R}} \times S^{d-1}$ and that its total mass is given by

$$
\left\|\mathscr{V}_{0}\right\|\left(\mathbf{R}^{d} \times S^{d-1}\right)=\left\langle\mathscr{V}_{0} ; 1\right\rangle=\lim _{\varepsilon \downarrow 0}\left\langle\mathscr{V}_{\varepsilon}^{\prime} ; 1\right\rangle \stackrel{(2.34)}{=} \lim _{\varepsilon \downarrow 0}\left\langle\mathscr{V}_{\varepsilon}^{\star}\left(a_{\varepsilon}\right) ; 1\right\rangle=S
$$

The last identity follows from the second estimate of Proposition 2.1.a. Taking into account $\mathscr{V}^{\star}\left(a_{\varepsilon}\right)-\mathscr{V}_{\varepsilon}^{\prime} \rightarrow 0$, we have established part (a) of the theorem.

Next, let us study the compactness properties of the family $T_{\varepsilon}=\left(\varepsilon-t_{\varepsilon}\right) / 2 \varepsilon$. These functions are compactly supported in $\overline{B_{R}}$ and by Proposition 2.1.a the sequence $\left(T_{\varepsilon}\right)$ is uniformly bounded in $B V\left(\mathbf{R}^{d}\right)$. Hence, up to a second extraction, we may assume that $\left(T_{\mathcal{\varepsilon}}\right)$ (weakly) converges in $B V\left(\mathbf{R}^{d}\right)$ towards a function $T \in B V\left(\mathbf{R}^{d}\right)$ which is compactly supported in $\overline{B_{R}}$. Moreover, by Proposition 2.1.a, $\mathscr{H}^{d}\left(\Omega_{\varepsilon}\right) \sim S \varepsilon$ and since $\left\{y \in \mathbf{R}^{d}: T_{\mathcal{E}}(y) \notin\{0,1\}\right\} \subset \Omega_{\varepsilon}$, we have $T \in\{0,1\}$ almost everywhere in $\mathbf{R}^{d}$. Therefore, there exists a set of finite perimeter $O_{0} \subset \overline{B_{R}}$ such that

$$
T_{\varepsilon} \rightarrow T=\mathbf{1}_{O_{0}} \text { weakly in } B V\left(\mathbf{R}^{d}\right) \text { as } \varepsilon \downarrow 0 .
$$

(For properties of BV functions and sets of finite perimeter, we refer to the books by Ambrosio, Fusco and Palara [3] or Evans and Gariepy [5]).

Now let us consider $\psi \in C_{c}\left(\mathbf{R}^{d}, \mathbf{R}^{d}\right)$ and let us apply Proposition 2.1.c with $\varphi(y, n)=$ $\psi(y) \cdot n$. Passing to the limit $\varepsilon \downarrow 0$, we get

$$
\int_{\mathbf{R}^{d}} \nabla \mathbf{1}_{O_{0}} \cdot \psi=-\left\langle\Lambda \mathscr{V}_{0} ; \psi\right\rangle, \quad \text { for every } \psi \in C_{c}\left(\mathbf{R}^{d}, \mathbf{R}^{d}\right) .
$$

Hence, $\Lambda \mathscr{V}_{0}=-\nabla \mathbf{1}_{O_{0}}$. This identity uniquely defines the limit $T=\mathbf{1}_{O_{0}}$, so the second extraction was not necessary.

This establishes part (b) and ends the proof of Theorem 1.1. 
3 Construction of a recovery family in the smooth case. Proof of Theorem $\mathbf{1 . 3}$

We consider an energy $\mathscr{F}(\sigma, \tau)=\int_{\tau \neq 0} f(\sigma, \tau)$ with stored energy function $f \in$ $C\left(\mathbf{R}^{d} \times, \mathbf{R}^{d}, \mathbf{R}_{+}\right)$. We assume that $f$ satisfies (1.1) and (1.6), (1.7), namely: $f \equiv 0$ on the sphere $\mathbb{S}^{d-1}=\left\{(e, e): e \in S^{d-1}\right\}, f$ is of class $C^{2}$ in some neighborhood $\mathscr{N}$ of $\mathbb{S}^{d-1}$ in $\mathbf{R}^{d} \times \mathbf{R}^{d}$ and $f$ is invariant under change of orthonormal coordinates in this neighborhood, i.e. $f\left(Q v_{1}, Q v_{2}\right)=f\left(v_{1}, v_{2}\right)$ for every $\left(v_{1}, v_{2}\right) \in \mathscr{N}$ and every (orientation preserving) $Q \in \mathrm{SO}(d)$.

The symmetry assumption and the minimality of $f$ on $\mathbb{S}^{d-1}$ yield a special structure for the Hessian matrix of $f$ on $\mathbb{S}^{d-1}$. This structure is described in the following lemma whose proof is postponed to Appendix C

Lemma 3.1 Let $e \in S^{d-1}$ and $b_{1}, \cdots, b_{d-1} \in S^{d-1}$ such that $\left(b_{1}, \cdots, b_{d-1}, e\right)$ is an orthonormal basis of $\mathbf{R}^{d}$. We define an orthonormal basis of $\mathbf{R}^{d} \times \mathbf{R}^{d}$ as

$$
\mathscr{B}=\left(\left(b_{1}, 0\right), \cdots,\left(b_{d-1}, 0\right),\left(0, b_{1}\right), \cdots,\left(0, b_{d-1}\right),(e, 0),(0, e)\right) .
$$

Then there exists $a \geq 0$ and $a 2 \times 2$ non-negative symmetric matrix $L$ which do not depend on e, such that, in the basis $\mathscr{B}$, the Hessian matrix of $f$ at point $(e, e)$ reads

$$
D^{2} f(e, e)=\left(\begin{array}{ccc}
a \mathbf{I}_{d-1} & -a \mathbf{I}_{d-1} & 0 \\
-a \mathbf{I}_{d-1} & a \mathbf{I}_{d-1} & 0 \\
0 & 0 & L
\end{array}\right)
$$

$\left(\mathbf{I}_{d-1}\right.$ denotes the identity matrix of size $(d-1) \times(d-1)$.)

Moreover, if (1.2) also holds ( $f \geq \kappa f_{0}$ for some $\left.\kappa>0\right)$, then a and L are positive.

Now let us establish Theorem 1.3. Let $O_{0} \subset \mathbf{R}^{d}$ be a smooth bounded open subset of $\mathbf{R}^{d}$, let $v_{0}$ the outward unit normal to $O_{0}$ and let $\Sigma_{0}:=\partial O_{0}$. We introduce the mapping

$$
\psi: \Sigma_{0} \times \mathbf{R} \rightarrow \mathbf{R}^{d}, \quad \psi(x, s):=x+s v_{0}(x) .
$$

There exists $\varepsilon_{\star}>0$ such that $\psi$ is a smooth (and bi-Lipschitz) diffeomorphism from $\Sigma_{0} \times\left(-\varepsilon_{\star}, \varepsilon_{\star}\right)$ onto its range $\Omega_{\star}$. The inverse mapping is given by $\Psi^{-1}=$ $\left(\pi_{0}, Z\right)$ where $\pi_{0}$ is the orthogonal projection on $\Sigma_{0}$ and where $Z(y):=d\left(y, O_{0}\right)-$ $d\left(y, \mathbf{R}^{d} \backslash O_{0}\right)$ is the signed distance function to $\Sigma_{0}$.

Let us extend $v_{0}$ as a mapping $n_{0}: \Omega_{\star} \rightarrow S^{d-1}$ by $n_{0}(y)=v_{0}\left(\pi_{0}(y)\right)=\nabla Z(y)$. We are going to build a quasi optimal family $\left\{a_{\varepsilon}\right\}=\left\{\left(\sigma_{\varepsilon}, \nabla t_{\varepsilon}, \Omega_{\varepsilon}\right)\right\}$ such that $\sigma_{\varepsilon}$ and $\nabla t_{\varepsilon}$ are the restrictions to $\Omega_{\varepsilon}$ of vector fields $\sigma$ and $\nabla t$ defined on $\Omega_{\star}$.

For symmetry reasons, we look for vector fields $\sigma$ and $\nabla t$ which are collinear to $n_{0}$ in $\Omega_{\star}$ and equal to $v_{0}$ on $\Sigma_{0}$. Under this ansatz the only possibility for the divergence free vector field $\sigma: \Omega_{\star} \rightarrow \mathbf{R}^{d}$ is to set

$$
\sigma(y):=\left(\operatorname{det}\left[\mathbf{I}_{d}+Z(y) D v_{0}\left(\pi_{0}(y)\right)\right]\right)^{-1} n_{0}(y) \quad \text { for every } y \in \Omega_{\star} .
$$

Let us check that $\sigma$ is divergence free in $\Omega_{\star}$. Equivalently, we have to show that $\int_{\Omega_{\star}} \nabla \varphi \cdot \sigma$ vanishes for every $\varphi \in \mathscr{D}\left(\Omega_{\star}\right)$. We perform the change of variable 
$y=\Psi(x, s)$. The Jacobian determinant of $\Psi$ is $J_{\Psi}(x, s)=\operatorname{det}\left[\mathbf{I}_{d}+s D v_{0}(x)\right]$ and we indeed obtain,

$$
\int_{\Omega_{\star}} \nabla \varphi(y) \cdot \sigma(y) d y=\int_{\Sigma_{0}}\left\{\int_{-\varepsilon_{\star}}^{\varepsilon_{\star}}\left[v_{0}(x) \cdot \nabla\right] \varphi\left(x+s v_{0}(x)\right) d s\right\} d \mathscr{H}^{d-1}(x)=0 .
$$

Then we set

$$
t(y):=\left(1+\frac{\alpha\left(\pi_{0}(y)\right)}{2} Z(y)\right) Z(y),
$$

where $\alpha \in C^{1}\left(\Sigma_{0}\right)$ has to be optimized. The function $\alpha$ being given, there exists $\varepsilon_{\star}^{\prime} \in\left(0, \varepsilon_{\star}\right)$ such that $\Phi_{\alpha}: y \mapsto\left(\pi_{0}(y), t(y)\right)$ is a smooth diffeomorphism from $\Sigma+B_{\varepsilon_{\star}^{\prime}}$ onto its range. Expanding the inverse of $\Phi_{\alpha}$ with respect to $\alpha$ around $\Phi_{0}^{-1}=\Psi$, we see that

$$
\Phi_{\alpha}^{-1}(x, s)=x+\left(1-\frac{\alpha(x)}{2} s+o(s)\right) s v_{0}(x)
$$

with $o(s) \rightarrow 0$ uniformly in $x$ as $s \rightarrow 0$. Moreover, lowering $\varepsilon_{\star}^{\prime}$ if necessary, we have for $\varepsilon \in\left(0, \varepsilon_{\star}^{\prime} / 2\right)$,

$$
\Sigma_{0}+B_{(1-o(\varepsilon)) \varepsilon} \subset \Phi_{\alpha}^{-1}(\Sigma \times(-\varepsilon, \varepsilon)) \subset \Sigma_{0}+B_{(1+o(\varepsilon)) \varepsilon} .
$$

Now, for $\varepsilon \in\left(0, \varepsilon_{\star}^{\prime} / 2\right)$ we set $\Omega_{\varepsilon}:=\Phi_{\alpha}^{-1}(\Sigma \times(-\varepsilon, \varepsilon))$ and we define

$$
t_{\varepsilon}(y):=t(y) \quad \text { for } y \in \overline{\Omega_{\varepsilon}} .
$$

By construction, $\partial \Omega_{\varepsilon}$ splits into two disjoint components $\partial \Omega_{\varepsilon}^{ \pm}$with $\partial \Omega_{\varepsilon}^{-} \subset O_{0}$, $\partial \Omega_{\mathcal{\varepsilon}}^{+} \subset \mathbf{R}^{d} \backslash O_{0}$ and we have $\pm t_{\varepsilon}=\varepsilon$ on $\partial \Omega_{\varepsilon}^{ \pm}$. We then extend $t_{\varepsilon}$ by continuity as

$$
t_{\varepsilon}:= \begin{cases}-\varepsilon & \text { in } O_{0} \backslash \Omega_{\varepsilon} \\ \varepsilon & \text { in } \mathbf{R}^{d} \backslash\left[\Omega_{\varepsilon} \cup O_{0}\right]\end{cases}
$$

Eventually, we set

$$
\sigma_{\varepsilon}(y):= \begin{cases}\sigma(y) & \text { if } y \in \Omega_{\varepsilon} \\ 0 & \text { if } y \in \mathbf{R}^{d} \backslash \Omega_{\varepsilon}\end{cases}
$$

By construction, we have $a_{\varepsilon}:=\left(\sigma_{\varepsilon}, \nabla t_{\varepsilon}, \Omega_{\varepsilon}\right) \in \mathscr{A}_{\varepsilon}(R+O(\varepsilon))$ where by definition, $R=\max \left\{|x| ; x \in \Sigma_{0}\right\}$. Writing $y=\Psi(x, s)$, we compute the expansions

$$
\begin{aligned}
\sigma_{\varepsilon}\left(x+s v_{0}(x)\right) & =v_{0}(x)+s h_{0}(x) v_{0}(x)+O\left(s^{2}\right), \\
\nabla t_{\varepsilon}\left(x+s v_{0}(x)\right) & =v_{0}(x)+s \alpha(x) v_{0}(x)+O\left(s^{2}\right),
\end{aligned}
$$

where $h_{0}(x)=-\nabla \cdot n_{0}(x)$ is the scalar mean curvature of $\Sigma_{0}$. Taking into account Lemma 3.1, we have for every $y=\Phi_{\alpha}^{-1}(x, s) \in \Omega_{\varepsilon}$,

$$
f\left(\sigma_{\varepsilon}(y), \nabla t_{\varepsilon}(y)\right)=\frac{s^{2}}{2}\left(h_{0}(x), \alpha(x)\right)^{T} L\left(h_{0}(x), \alpha(x)\right)+o\left(s^{2}\right) .
$$


Optimizing with respect to $\alpha(x)$, we get $\alpha(x)=-\left(L_{1,2} / L_{2,2}\right) h_{0}(x)$ which yields

$$
f\left(\sigma_{\varepsilon}(y), \nabla t_{\varepsilon}(y)\right)=h_{0}^{2}(x) \frac{\operatorname{det} L}{2 L_{2,2}} s^{2}+o\left(s^{2}\right) .
$$

Taking into account (3.3), the energy of $a_{\varepsilon}$ then expands as

$$
\mathscr{F}\left(a_{\varepsilon}\right)=\frac{\operatorname{det} L}{3 L_{2,2}} \mathscr{W}\left(\Sigma_{0}\right) \varepsilon^{3}+o\left(\varepsilon^{3}\right) .
$$

This is the expected expansion, however, the volume constraint is not exactly satisfied. We only have

$$
\mathscr{Q}\left(\sigma_{\varepsilon}, \nabla t_{\varepsilon}\right)=\int_{\Omega_{\varepsilon}} \sigma \cdot \nabla t=2 \varepsilon \mathscr{H}^{d-1}\left(\Sigma_{0}\right)+O\left(\varepsilon^{2}\right) .
$$

We recover the exact constraint $\mathscr{Q}\left(\sigma_{\varepsilon}^{\prime}, \nabla t_{\varepsilon}^{\prime}\right)=2 \varepsilon \mathscr{H}^{d-1}\left(\Sigma_{0}\right)$ by substituting $a_{\varepsilon}^{\prime}=$ $\left(\sigma_{\varepsilon}^{\prime}, \nabla t_{\varepsilon}^{\prime}, \Omega_{\varepsilon}^{\prime}\right)$ for $a_{\varepsilon}$ with

$$
\sigma_{\varepsilon}^{\prime}(y):=\sigma_{\varepsilon}\left(\left(1 / \lambda_{\varepsilon}\right) y\right), \quad t_{\varepsilon}^{\prime}(y):=\lambda_{\varepsilon} t_{\varepsilon}\left(\left(1 / \lambda_{\varepsilon}\right) y\right), \quad \Omega_{\varepsilon}^{\prime}:=\lambda_{\varepsilon} \Omega_{\varepsilon},
$$

where the magnification factor is defined as

$$
\lambda_{\varepsilon}:=\left[\frac{\mathscr{Q}\left(\sigma_{\varepsilon}, \nabla t_{\varepsilon}\right)}{2 \varepsilon \mathscr{H}^{d-1}(\Sigma)}\right]^{1 / d}=1+O(\varepsilon)
$$

The convergence properties of $\left\{\mathscr{V}_{\varepsilon}^{\star}\left(a_{\varepsilon}^{\prime}\right)\right\}$ towards $\mathscr{V}\left(\Sigma_{0}, v_{0}\right)$ and of the sets $\left\{\left[t_{\varepsilon}^{\prime}=\right.\right.$ $-\varepsilon]\}$ towards $O_{0}$ are straightforward by construction. This ends the proof of Theorem 1.3.

\section{Lower bound. Proof of Theorem 1.2}

In this Section, we assume that the stored energy function $f$ satisfies (1.1), (1.2) and (1.6), (1.7) and we consider an oriented $(d-1)$-varifold $\mathscr{V}_{0}$ and a sequence $\left(a_{\varepsilon_{k}}\right)_{k \geq 0}$ with $\varepsilon_{k} \downarrow 0$ such that $a_{\varepsilon_{k}} \in \mathscr{A}_{\varepsilon_{k}}(R, S)$ for some $R, S>0$ and $\mathscr{V}_{\varepsilon_{k}}\left(a_{\varepsilon_{k}}\right) \rightarrow \mathscr{V}_{0}$ as $\varepsilon_{k} \downarrow 0$.

The lower bound result being obvious in the case $\liminf _{k} \mathscr{F}\left(a_{\varepsilon_{k}}\right) / \varepsilon_{k}^{3}=+\infty$, we assume without loss of generality, that there exists $E_{0}>0$ such that

$$
\mathscr{F}_{0}\left(a_{\varepsilon_{k}}\right) \leq E_{0} \varepsilon_{k}^{3}, \quad \text { for every } k \geq 0
$$

Consequently, the constructions and estimates of Proposition 2.1 and Theorem 1.1 apply to the sequence $\left\{a_{\varepsilon_{k}}\right\}$ and $\mathscr{V}_{0}$. In particular $\mathscr{V}_{0} \in \mathscr{A}_{0}(R, S)$ and by Appendix D $\mathscr{V}_{0}=\mathscr{V}\left(\Sigma_{0}, v_{0}, \theta_{0}^{+}, \theta_{0}^{-}\right)$is an oriented integer rectifiable $(d-1)$-varifold which admits a $L^{2}$-generalized mean curvature. To lighten notation, we drop the subscripts $k$ in the sequence $\varepsilon_{k}$. We use the notation of Section 2 for the objects constructed along the proof of Proposition 2.1: $u_{\varepsilon}, O_{\varepsilon}, \Sigma_{\varepsilon}, v_{\varepsilon}$ and the corresponding rescaled objects $u_{(\varepsilon)}, O_{(\varepsilon)}, \Sigma_{(\varepsilon)}, v_{(\varepsilon)}$, etc. 
By lower semi-continuity of the Willmore energy (see (D.6)) we have

$$
\mathscr{W}\left(\mathscr{V}_{0}\right) \leq \liminf _{\varepsilon \downarrow 0} \mathscr{W}\left(\Sigma_{\varepsilon}\right) .
$$

However, there is little hope for deducing the lower bound of Theorem 1.2 from this inequality. Indeed, the mean curvature $h_{\varepsilon}$ of $\Sigma_{\varepsilon}$ is loosely and non locally related to the data $a_{\varepsilon}$ through the harmonic function $u_{\varepsilon}$. Remark that $u_{\varepsilon}$ does not even depend on $\sigma_{\varepsilon}$. In short (and omitting cut-off issues) our strategy is the following.

$i$. In Section 4.1, we define an approximate mean curvature $\hat{h}_{\varepsilon}(x)$ as the dot product of $\sigma_{\varepsilon}$ with a particular test function $\zeta$ compactly supported in $x+\varepsilon D_{1}^{0}(v(x))$. We also establish that $\hat{h}_{\varepsilon}$ is indeed an approximation of $h_{\varepsilon}$ in a weak sense. We deduce the inequality

$$
\mathscr{W}\left(\mathscr{V}_{0}\right) \leq \liminf _{\varepsilon \downarrow 0} \int_{\Sigma_{\varepsilon}}\left|\hat{h}_{\varepsilon}\right|^{2} d \mathscr{H}^{d-1} .
$$

ii. In Section 4.2, we introduce local minimization problems of the form

$$
c_{\zeta, \eta}(f):=\inf \left[\mathscr{F}\left(\sigma^{\#}, \nabla t^{\#}, D_{1-2 \xi}^{2 \xi}\left(e_{d}\right)\right) /\left|\hat{h}^{\#}\right|^{2}\right],
$$

where the infimum ranges over all admissible vector fields $\sigma^{\#}, \nabla t^{\#}$ with approximate mean curvature $\hat{h}^{\#} \in(-\eta, \eta) \backslash\{0\}$ at $x=0$. With this notation, we deduce from (4.1)

$$
\begin{aligned}
& c_{\zeta, \eta}(f) \mathscr{W}\left(\mathscr{V}_{0}\right) \leq \\
& \quad \liminf _{\varepsilon \downarrow 0} \frac{1}{\varepsilon^{3}} \int_{\Sigma_{\varepsilon} \cap\left[\hat{h}_{\varepsilon} \mid<\eta / \varepsilon\right]} \mathscr{F}\left(\sigma_{\varepsilon}, \nabla t_{\varepsilon}, x+\varepsilon D_{1-2 \xi}^{2 \xi}\left(v_{\varepsilon}(x)\right)\right) d \mathscr{H}^{d-1}(x) .
\end{aligned}
$$

We then show that the above integral is bounded by $[1+s(\eta)] \mathscr{H}^{d-1}\left(D_{1}^{\prime}\right) \mathscr{F}\left(a_{\varepsilon}\right)$ with $s(\eta) \rightarrow 0$ as $\eta \downarrow 0$ and get

$$
c_{\zeta, \eta}(f) \mathscr{W}\left(\mathscr{V}_{0}\right) \leq[1+s(\eta)] \mathscr{H}^{d-1}\left(D_{1}^{\prime}\right) \liminf _{\varepsilon \downarrow 0} \frac{\mathscr{F}\left(a_{\varepsilon}\right)}{\varepsilon^{3}}
$$

We study the local problems (4.2) and establish

$$
\liminf _{\eta \downarrow 0} c_{\zeta, \eta}(f) \geq \mathscr{H}^{d-1}\left(D_{1}^{\prime}\right) c_{0, \zeta}(f),
$$

where $c_{0, \zeta}(f)$ depends explicitly on $f$ and on the test function $\zeta$. Eventually, optimizing wit respect to $\zeta$ we obtain $\sup _{\zeta} c_{0, \zeta}(f)=c_{0}(f)$ which yields the desired lower bound.

The above description leaves aside the following technical difficulties: 1/ we are only able to define the approximate mean curvature in a good set, so we need to perform a cut-off and control the volume of the bad set; $2 /$ we need also to control the $(d-1)$-volumes of the sets $\Sigma_{\varepsilon} \cap\left[\left|\hat{h}_{\varepsilon}\right| \geq \eta / \varepsilon\right] ; 3 /$ for compactness issues we introduce a small parameter $\alpha>0$ and (with obvious notation), we substitute

$$
\mathscr{F}\left(\sigma^{\#}, \nabla t^{\#}, D_{1-2 \xi}^{2 \xi}\right)+\alpha \int_{B_{3} \cap \Omega^{\#}}\left[f_{0}\left(\sigma^{\#}, \nabla t^{\#}\right)+\left|\nabla u^{\#}-\nabla t^{\#}\right|^{2}\right]
$$

for $\mathscr{F}\left(\sigma^{\#}, \nabla t^{\#}, D_{1-2 \xi}^{2 \xi}\right)$ in the local optimization problem. 
4.1 Definition and properties of the approximate mean curvature.

We already know that $\sigma_{\varepsilon}$ approximates $v_{\varepsilon}$, so a naive way to build an approximation of the mean curvature on $\Sigma_{\varepsilon}$ by means of the data $\left(\sigma_{\varepsilon}, \nabla t_{\varepsilon}\right)$ is to set $\hat{h}_{\varepsilon}(x):=\operatorname{div}_{\Sigma_{\varepsilon}} \sigma_{\varepsilon}(x)$. However, the regularity of $\sigma_{\varepsilon}$ is too weak to provide a robust definition under sole energy bounds. The next idea consists in using a weak notion for the mean curvature where, thanks to integration by parts, the space derivatives on $\sigma_{\varepsilon}$ are transferred to a test function. The relevant tool for this is the tangential Green formula.

Let us consider a smooth compact hypersurface $\Gamma \subset \mathbf{R}^{d}$ without boundary, with normal $v$ and mean curvature $h$. Let $X \in C^{1}\left(\Gamma, \mathbf{R}^{d}\right)$ of the form $X(x)=\varphi(x) v(x)$ and let us extend it as a mapping $X \in C_{c}^{1}\left(\mathbf{R}^{d}, \mathbf{R}^{d}\right)$. For such a test vector field, the tangential Green formula (D.2) reads,

$$
\int_{\Gamma} \varphi h v d \mathscr{H}^{d-1}=-\int_{\Gamma} \nabla_{\Gamma} \varphi d \mathscr{H}^{d-1} \quad \text { for every } \varphi \in \mathscr{D}\left(\mathbf{R}^{d}\right) .
$$

Assume for simplicity that $0 \in \Gamma$ with $v(0)=e_{d}$ and let us localize the above identity around 0 . For this, we introduce a function of the form

$$
\zeta\left(y^{\prime}, y_{d}\right):=\chi_{/ /}\left(\left|y^{\prime}\right|\right) \chi_{\perp}\left(y_{d}\right)
$$

with $\chi_{/ /}, \chi_{\perp} \in \mathscr{D}\left(\mathbf{R}, \mathbf{R}_{+}\right)$even and such that $\chi_{\perp}(0) \int_{\mathbf{R}^{d-1}} \chi_{/ /}\left(\left|y^{\prime}\right|\right) d y^{\prime}=1$. Using the test function $\varphi(y)=\eta^{1-d} \zeta((1 / \eta) y)$ in (4.3), taking the scalar product with $v(0)=e_{d}$ and sending $\eta$ to 0 , we obtain after some Taylor expansions,

$$
h(0)=\lim _{\eta \downarrow 0} \frac{1}{\eta} \int_{\Gamma_{(\eta)}} \chi_{\perp}\left(z_{d}\right) v_{(\eta)}(z) \cdot \nabla \chi_{/ /}\left(\left|z^{\prime}\right|\right) d \mathscr{H}^{d-1}(z) .
$$

where $\Gamma_{(\eta)}$ is the hypersurface $(1 / \eta) \Gamma$ and $v_{(\eta)}(z):=v(\eta z)$.

We mimic this formula for defining an approximate mean curvature on $\Sigma_{\varepsilon}$. For this, we choose $\eta=\varepsilon$ and we substitute the vector field $\sigma_{(\varepsilon)}$ for the outward unit normal $v_{(\eta)}=v_{(\varepsilon)}$. This substitution closely connects the approximate curvature to the data. We also thicken the domain of integration by substituting the cylinder $D_{1}^{0} \subset \mathbf{R}^{d}$ for the piece of hypersurface $\Sigma_{(\varepsilon)} \cap \operatorname{supp} \zeta$. The precise construction is detailed below.

Definition 4.1 Let $\chi_{/ /}, \chi_{\perp} \in W^{1, \infty}\left(\mathbf{R}, \mathbf{R}_{+}\right)$be two nonnegative, even cut-off functions which are compactly supported in $(-1,1)$ and satisfy

$$
\left(\int_{\mathbf{R}^{d-1}} \chi_{/ /}\left(\left|y^{\prime}\right|\right) d \mathscr{H}^{d-1}\left(y^{\prime}\right)\right)\left(\int_{\mathbf{R}} \chi_{\perp}(s) d s\right)=1 .
$$

We define a Lipschitz test function $\zeta \in W^{1, \infty}\left(\mathbf{R}^{d} \times S^{d-1}, \mathbf{R}_{+}\right)$, by setting

$$
\zeta:=\zeta_{/ /} \zeta_{\perp} \quad \text { with } \quad \zeta_{\perp}(y ; \bar{n}):=\chi_{\perp}(y \cdot \bar{n}), \quad \zeta_{/ /}(y ; \bar{n}):=\chi_{/ /}\left(\left|\pi_{\bar{n}} y\right|\right)
$$


Notice that for $\bar{n} \in S^{d-1}$, the function $y \mapsto \zeta(y ; \bar{n})$ is compactly supported in $D_{1}^{0}(\bar{n})$. In the sequel, we fix $\xi=\xi(\zeta) \in(0,1 / 4)$ such that

$$
\operatorname{supp} \zeta(\cdot ; \bar{n}) \subset D_{1-2 \xi}^{2 \xi}(\bar{n}) \quad \text { for } \bar{n} \in S^{d-1} .
$$

Notice also that

$$
\int_{\mathbf{R}^{d}} \zeta(y ; \bar{n}) d y=1 .
$$

Definition 4.2 Let us introduce a small parameter $\eta \in(0, \xi / 2)$ and let us set

$$
\beta_{\eta}:=\min \left(\beta_{\dot{\xi}, 1}^{\star}(\xi, \eta), \beta_{\dot{\xi}, 3}^{\star}(\xi / 2, \eta)\right),
$$

where the functions $\beta_{\dot{\phi}, 1}^{\star}$ and $\beta_{\dot{\phi}, 3}^{\star}$ are given in Lemma 2.4 and Lemma 2.6. Using the notation of Lemma 2.7, we define the two bad sets (union of bad balls),

$$
\mathscr{U}_{(\varepsilon), \eta}:=\left[U_{(\varepsilon)}^{\star} \cup U_{(\varepsilon), \beta_{\eta}}\right]+B_{\sqrt{2}} \subset \mathscr{U}_{(\varepsilon), \eta}^{\prime}:=\mathscr{U}_{(\varepsilon), \eta}+B_{\sqrt{2}},
$$

and the corresponding good sets

$$
\mathscr{G}_{(\varepsilon), \eta}:=\mathbf{R}^{d} \backslash \mathscr{U}_{(\varepsilon), \eta}, \quad \supset \quad \mathscr{G}_{(\varepsilon), \eta}^{\prime}:=\mathbf{R}^{d} \backslash \mathscr{U}_{(\varepsilon), \eta}^{\prime} .
$$

Next, we define a cut-off function $\chi_{(\varepsilon), \eta} \in \mathscr{D}\left(\mathbf{R}^{d},[0,1]\right)$ satisfying

$$
\chi_{(\varepsilon), \eta} \equiv 1 \text { on } \mathscr{G}_{(\varepsilon), \eta}^{\prime}, \quad \chi_{(\varepsilon), \eta} \equiv 0 \text { on } \mathscr{U}_{(\varepsilon), \eta}, \quad\left\|\nabla \chi_{(\varepsilon), \eta}\right\|_{\infty} \leq 1
$$

The approximate (scalar) mean curvature of $\Sigma_{\varepsilon}$ at some point $x \in \Sigma_{\varepsilon}$ is defined as

$$
\hat{h}_{\varepsilon}(x):=\frac{\chi_{(\varepsilon), \eta}(\varepsilon x)}{\varepsilon} \int_{\mathbf{R}^{d}} \sigma_{\varepsilon}(x+\varepsilon z) \cdot \pi_{v_{\varepsilon}(x)} \nabla_{y} \zeta\left(z ; v_{\varepsilon}(x)\right) d z .
$$

Eventually, the approximate mean curvature is defined as $\hat{H}_{\varepsilon}(x):=\hat{h}_{\varepsilon}(x) v_{\varepsilon}(x)$.

Remark 4.1 To lighten the notation in the above definitions we did not emphasized the dependencies in $\zeta$ and $\eta$. Below, the main computations are carried out for a fixed test function $\zeta$ and a fixed parameter $\eta$. Only at the end, we send $\eta$ to 0 and we optimize the lower bound with respect to $\zeta$.

The next proposition states that $\hat{h}_{\varepsilon}$ approximates $h_{\varepsilon}$, at least in a weak sense in $L^{2}\left(\Sigma_{\varepsilon}, \mathscr{H}^{d-1}\right)$

Proposition 4.1 Let $\hat{h}_{\varepsilon}$ be given by Definition 4.2:

a) We have the following uniform bound in $\varepsilon$,

$$
\int_{\Sigma_{\varepsilon}}\left|\hat{h}_{\varepsilon}\right|^{2} d \mathscr{H}^{d-1} \leq C_{\dot{\phi}}(\zeta, \eta)
$$

b) Moreover, for every $\varphi \in C\left(\mathbf{R}^{d}\right)$,

$$
\int_{\Sigma_{\varepsilon}} \varphi\left[\hat{h}_{\varepsilon}-h_{\varepsilon}\right] v_{\varepsilon} d \mathscr{H}^{d-1} \stackrel{\varepsilon \downarrow 0}{\longrightarrow} 0 .
$$


Proof Let us fix $\varepsilon \in(0,1]$ and for $x \in \Sigma_{(\varepsilon)}$, let us set

$$
\hat{h}_{(\varepsilon)}(x):=\varepsilon \hat{h}_{\varepsilon}(\varepsilon x) .
$$

We now drop the subscripts $(\varepsilon)$ : we write $\Sigma=\Sigma_{(\varepsilon)}, U^{\star}=U_{(\varepsilon)}^{\star}, \chi_{\eta}=\chi_{(\varepsilon), \eta}$, etc. In rescaled variables, we have for $x \in \Sigma$,

$$
\hat{h}(x)=\chi_{\eta}(x) \int_{\mathbf{R}^{d}} \sigma(x+y) \cdot\left(\zeta_{\perp} \nabla_{y} \zeta_{/ /}\right)(y ; v(x)) d y .
$$

Recall that in the above integral we can reduce the domain of integration to $D_{1-2 \xi}^{2 \xi}(v(x))$.

We start with a few remarks. Let us consider the piece of hypersurface,

$$
\Gamma_{\eta}:=\Sigma \cap \operatorname{supp} \chi_{\eta}
$$

By definition of $\chi_{\eta}$, we have supp $\chi_{\eta}+B_{\sqrt{2}} \subset U^{\star}$ so that $\Sigma \cap\left(\operatorname{supp} \chi_{\eta}+B_{\sqrt{2}}\right) \subset$ $\Sigma^{0}$. In particular, $\Gamma_{\eta} \subset \Sigma_{0}$ and $v(x)=n(x)$ on $\Gamma_{\eta}$

By definition of $\beta_{\eta}$, Lemma 2.4 and Lemma 2.5 apply to any point of $\Gamma_{\eta}$. Following the notation of these lemmas, we define for every $x \in \Gamma_{\eta}$,

$$
D_{\text {int }}(x):=x+D_{1-2 \xi}^{2 \xi}(n(x)) \quad \subset \quad D(x):=x+D_{1-\xi}^{\xi}(n(x)) \quad \subset \quad \Omega .
$$

The inequalities (2.11) and (2.13) apply at $x$ and moreover, $\Sigma \cap D(x)=\Sigma^{0} \cap$ $D(x)$. Eventually, since $\eta<\xi / 2$, Lemma 2.4 implies $|t| \leq 1-\xi / 2$ in $D(x)$; so Lemma 2.6 apply to any element $y \in D(x), x \in \Gamma_{\eta}$.

(a) We have to estimate the quantity

$$
\int_{\Sigma_{\varepsilon}} \hat{h}_{\varepsilon}^{2} d \mathscr{H}^{d-1}=\varepsilon^{d-3} \int_{\Gamma_{\eta}} \hat{h}^{2} d \mathscr{H}^{d-1} .
$$

Let $x \in \Gamma_{\eta}$ and let $\psi_{x}$ be the harmonic function provided by Lemma 2.5.c. Since $\nabla \psi_{x}=\pi_{n(x)} \nabla \psi_{x}$ and $\psi_{x}$ is harmonic, we have

$$
\int_{\mathbf{R}^{d}} \nabla \psi_{x}(y) \cdot \nabla_{y} \zeta_{/ /}(y ; n(x)) \zeta_{\perp}(y ; n(x)) d y=\int_{\mathbf{R}^{d}} \nabla \psi_{x}(y) \cdot \nabla_{y} \zeta(y ; n(x)) d y=0 .
$$

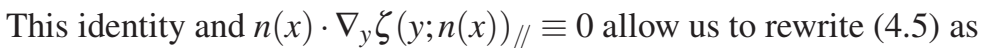

$$
\hat{h}(x)=\chi_{\eta}(x) \int_{\mathbf{R}^{d}}\left[\sigma(x+y)-n(x)-\nabla \psi_{x}(x+y)\right] \cdot\left(\zeta_{\perp} \nabla_{y} \zeta_{/ /}\right)(y ; n(x)) d y .
$$

Then (2.13) and the Cauchy-Schwarz inequality yield $|\hat{h}|^{2}(x) \leq C(\zeta) \mathscr{E}(D(x))$. Integrating on $\Gamma_{\eta}$ and using Fubini, we obtain

$$
\int_{\Gamma_{\eta}}|\hat{h}|^{2} d \mathscr{H}^{d-1} \leq C(\zeta) \int_{\Omega^{1-\xi / 2}}\left[f_{0}(\sigma, \nabla t)+|\nabla u-\nabla t|^{2}\right](y) q^{\xi}(y) d y,
$$

with the notation $\Omega^{1-\xi / 2}:=[|t|<1-\xi / 2]$ and

$$
q^{\xi}(y):=\int_{\Gamma_{\eta}} \theta^{\xi}(y-x, n(x)) d \mathscr{H}^{d-1}(x) .
$$


where $\theta^{\xi}(\cdot, n(x))$ is the characteristic function of $D_{1-\xi}^{\xi}(n(x))$.

By inequality (2.14) of Lemma 2.6, we have $\left|q^{\xi}(y)\right| \leq C(\eta)$. Hence,

$\varepsilon^{d-3} \int_{\Gamma_{\eta}}|\hat{h}|^{2} d \mathscr{H}^{d-1} \leq C(\zeta) \varepsilon^{d-3} \int_{\Omega}\left[f_{0}(\sigma, \nabla t)+|\nabla u-\nabla t|^{2}\right] \stackrel{\text { (Lemma 2.2) }}{\leq} C_{\mathscr{q}}(\zeta)$.

This establishes Proposition 4.1.a.

(b) Let $\varphi \in C\left(\mathbf{R}^{d}\right)$. In scaled variables we have to bound the vector $\hat{Q}_{(\varepsilon)}[\varphi]-$ $Q_{(\varepsilon)}[\varphi] \in \mathbf{R}^{d}$, with

$$
\begin{aligned}
& \hat{Q}_{(\varepsilon)}[\varphi]:=\varepsilon^{d-2} \int_{\Sigma_{(\varepsilon)}} \varphi(\varepsilon x) \hat{h}_{(\varepsilon)}(x) n_{(\varepsilon)}(x) d \mathscr{H}^{d-1}(x), \\
& Q_{(\varepsilon)}[\varphi]:=\varepsilon^{d-2} \int_{\Sigma_{(\varepsilon)}} \varphi(\varepsilon x) h_{(\varepsilon)}(x) n_{(\varepsilon)}(x) d \mathscr{H}^{d-1}(x) .
\end{aligned}
$$

The scaling factors $\varepsilon^{d-2}$ come from $\mathscr{H}^{d-1}\left(\Sigma_{\varepsilon}\right)=\varepsilon^{d-1} \mathscr{H}^{d-1}\left(\Sigma_{(\varepsilon)}\right)$ and $h_{\varepsilon}(x)=$ $\varepsilon^{-1} h_{(\varepsilon)}\left(\varepsilon^{-1} x\right)$.

Let us drop again the subscripts $(\varepsilon)$. Recall that $\Sigma \subset B_{R / \varepsilon}$, so we can assume that $\varphi$ is compactly supported. We can in fact assume that $\varphi$ is Lipschitz continuous by density of $W^{1, \infty}\left(\overline{B_{R / \varepsilon}}\right)$ in $C\left(\overline{B_{R / \varepsilon}}\right)$. Indeed, $\varphi \mapsto(Q-\widetilde{Q})[\varphi]$ is linear and by part (a) and the bounds of Proposition 2.1.b,

$$
\begin{aligned}
|(\hat{Q}-Q)[\varphi]| \leq\|\varphi\|_{\infty} \sqrt{2 \varepsilon^{d-3} \int_{\Sigma}|\hat{h}|^{2}+|h|^{2} d \mathscr{H}^{d-1}} \sqrt{\varepsilon^{d-1} \mathscr{H}^{d-1}(\Sigma)} \\
\leq C_{\not}(\zeta, \eta)\|\varphi\|_{\infty} .
\end{aligned}
$$

Now, we claim that we can somehow assume that $\varphi$ is supported in $\mathscr{G}_{\eta}^{\prime}$. Let us introduce a cut-off function $\chi_{\eta}^{\prime} \in \mathscr{D}\left(\mathbf{R}^{d},[0,1]\right)$ satisfying

$$
\chi_{\eta}^{\prime} \equiv 1 \text { on } \mathscr{G}_{\eta}^{\prime \prime}, \quad \chi_{\eta}^{\prime} \equiv 0 \text { on } \mathscr{U}_{\eta}^{\prime}, \quad\left\|\nabla \chi_{\eta}^{\prime}\right\|_{\infty} \leq 1 .
$$

with the larger bad set and smaller good set:

$$
\mathscr{U}_{\eta}^{\prime \prime}:=\mathscr{U}_{\eta}^{\prime}+B_{\sqrt{2}}, \quad \mathscr{G}_{\eta}^{\prime \prime}:=\mathbf{R}^{d} \backslash \mathscr{U}_{\eta}^{\prime \prime} .
$$

We then define

$$
\varphi_{\eta}(y):=\chi_{\eta}^{\prime}((1 / \varepsilon) y) \varphi(y) .
$$

Since $\left|\varphi_{\eta}-\varphi\right|$ is supported in $\mathscr{U}_{\eta}^{\prime \prime}$ and bounded by $\|\varphi\|_{\infty}$, we have, proceeding as above and using (2.23):

$$
\begin{aligned}
& \left|(\hat{Q}-Q)\left[\varphi_{\eta}-\varphi\right]\right| \\
& \leq \sqrt{2 \varepsilon^{d-3} \int_{\Sigma}|\hat{h}|^{2}+|h|^{2} d \mathscr{H}^{d-1}} \sqrt{\varepsilon^{d-3} \mathscr{H}^{d-1}\left(\Sigma \cap \mathscr{U}_{\eta}^{\prime \prime}\right)}\|\varphi\|_{\infty} \varepsilon \\
& \leq C_{\phi}(\zeta, \eta)\|\varphi\|_{\infty} \varepsilon \stackrel{\varepsilon \downarrow 0}{\longrightarrow} 0 .
\end{aligned}
$$


Therefore, we may and do substitute $\varphi_{\eta}$ for $\varphi$ and assume $\varphi \in W^{1, \infty}\left(\mathbf{R}^{d}\right)$ in the proof of (b). Remark that albeit not explicitly written, $\varphi_{\eta}$ depends on $\varepsilon$. Let us summarize the properties of $\varphi_{\eta}$ :

$$
\begin{array}{cc}
\left\|\varphi_{\eta}\right\|_{\infty} \leq\|\varphi\|_{\infty}, & \operatorname{supp} \varphi_{\eta} \subset \mathscr{G}_{\eta}^{\prime}, \\
\left\|\nabla \varphi_{\eta}\right\|_{L^{\infty}\left(\mathscr{G}_{\eta}^{\prime \prime}\right)} \leq\|\nabla \varphi\|_{\infty}, & \left\|\nabla \varphi_{\eta}\right\|_{L^{\infty}\left(\mathscr{U}_{\eta}^{\prime \prime}\right)} \leq \varepsilon^{-1}\|\varphi\|_{\infty}+\|\nabla \varphi\|_{\infty} .
\end{array}
$$

In particular $\chi_{\eta} \equiv 1$ on the support of $\varphi_{\eta}$ and we can drop the factor $\chi_{\eta}$ and replace $\Sigma$ by $\Gamma_{\eta} \subset \Sigma_{0}$ in the definitions of $Q_{(\varepsilon)}\left[\varphi_{\eta}\right]$ and $\hat{Q}_{(\varepsilon)}\left[\varphi_{\eta}\right]$.

In the sequel we write $Q$ and $\hat{Q}$ for $Q\left[\varphi_{\eta}\right]$ and $\hat{Q}\left[\varphi_{\eta}\right]$. Using formula (4.5), we rewrite $\hat{Q}$ as an integral over $(x, y) \in \Sigma \times \mathbf{R}^{d}$. Then, performing the change of variables $z=x+y \in \mathbf{R}^{d}, x \in \Gamma_{\eta}$ and using Fubini, we obtain, for $i=1, \cdots, d-1$,

$$
\hat{Q}_{i}=\varepsilon^{d-2} \int_{\mathbf{R}^{d}} \sigma(z) \cdot \hat{q}_{i}(z) d z,
$$

with the notation $\hat{Q}=\left(\hat{Q}_{1}, \cdots, \hat{Q}_{d}\right)$ and

$$
\hat{q}_{i}(z):=\int_{\Gamma_{\eta}} \varphi_{\eta}(\varepsilon x) n_{i}(x)\left[\zeta_{\perp} \nabla_{y} \zeta_{/ /}\right](z-x ; n(x)) d \mathscr{H}^{d-1}(x) .
$$

Here $n_{i}(x)$ denotes the $i$-th component of $n(x)$.

Next, having in mind the identities $\pi_{\bar{n}} \nabla_{y} \zeta_{/ /}(y ; \bar{n})=\nabla_{y} \zeta_{/ /}(y ; \bar{n})$ and $\pi_{\bar{n}} \nabla_{y} \zeta_{\perp}(y ; \bar{n})=$ 0 , we compute the expansion,

$$
\begin{aligned}
\pi_{n(x)} \nabla_{x}\left[\varphi_{\eta}(\varepsilon x) \zeta(z-x ; n(x))\right. & \left.n_{i}(x)\right] \\
& =a_{i}(x, z)+b_{i}(x, z)+\varphi_{\eta}(\varepsilon x) \pi_{n(x)} c_{i}(x, z),
\end{aligned}
$$

where:

$a$. The term $a_{i}(x, z)$ involves the gradient of the function $y \mapsto \zeta(y ; \bar{n})$,

$$
a_{i}(x, z):=-\varphi_{\eta}(\varepsilon x) n_{i}(x)\left[\zeta_{\perp} \nabla_{y} \zeta_{/ /}\right](z-x ; n(x)) .
$$

Remark that $a_{i}(x, z)$ is the negative of the integrand in (4.8).

$b$. The term $b_{i}(x, z)$ involves the gradient of $x \mapsto \varphi_{\eta}(\varepsilon x)$.

$$
b_{i}(x, z):=\varepsilon \zeta(z-x ; n(x)) n_{i}(x) \pi_{n(x)} \nabla \varphi_{\eta}(\varepsilon x) .
$$

$c$. The last term $c_{i}(x, z)$ involves the gradient of $n(x)$. It can be written on the following form,

$$
c_{i}(x, z)=\left.\sum_{k=1}^{d} \frac{\partial\left[\zeta(z-x ; \bar{n}) \bar{n}_{i}\right]}{\partial \bar{n}_{k}}\right|_{\bar{n}=n(x)} \nabla n_{k}(x)=\sum_{j=1}^{d} c_{i, j}(x ; z) \nabla n_{j}(x) .
$$

We have $\operatorname{supp}\left[c_{i, j}(\cdot ; z)\right] \subset D_{\sqrt{2}}(z)$ and $\left\|c_{i, j}\right\|_{\infty} \leq C(\zeta)$. 
Integrating (4.9) on $\Gamma_{\eta}$ and applying the Green formula (4.3) to the left hand side, we get,

$$
\begin{aligned}
\hat{q}_{i}(z)=- & \int_{\Gamma_{\eta}} a_{i}(x, z) d \mathscr{H}^{d-1}(z) \\
= & \int_{\Gamma_{\eta}} \varphi_{\eta}(\varepsilon x) \zeta(z-x ; n(x)) n_{i}(x) h(x) n(x) d \mathscr{H}^{d-1}(x) \\
& \quad+\int_{\Gamma_{\eta}} b_{i}(x, z) d \mathscr{H}^{d-1}(x)+\int_{\Gamma_{\eta}} \varphi_{\eta}(\varepsilon x) \pi_{n(x)} c_{i}(x, z) d \mathscr{H}^{d-1}(x) .
\end{aligned}
$$

Taking the dot product with $\sigma(z)=n(x)+[\sigma(z)-n(x)]$, integrating in $z \in \mathbf{R}^{d}$ and changing back the variables of integration to $y=z-x, x=x$, we obtain,

$$
\hat{Q}_{i}=\hat{Q}_{i, 1}+\hat{Q}_{i, 2}+\hat{Q}_{i, 3}+\hat{Q}_{i, 4}
$$

with

$$
\begin{aligned}
& \hat{Q}_{i, 1}:=\varepsilon^{d-2} \int_{\Gamma_{\eta}}\left[\int_{\mathbf{R}^{d}} \zeta(\cdot ; n(x))\right] h(x) \varphi_{\eta}(\varepsilon x) n_{i}(x) d \mathscr{H}^{d-1}(x) \\
& \hat{Q}_{i, 2}:=\varepsilon^{d-2} \int_{\Gamma_{\eta}}\left[\int_{\mathbf{R}^{d}} \zeta(\cdot ; n(x))(\sigma(x+\cdot)-n(x))\right] \cdot n(x) h(x) \varphi_{\eta}(\varepsilon x) n_{i}(x) d \mathscr{H}^{d-1}(x) \\
& \hat{Q}_{i, 3}:=\varepsilon^{d-1} \int_{\Gamma_{\eta}}\left[\int_{\mathbf{R}^{d}} \zeta(\cdot ; n(x)) \sigma(x+\cdot)\right]^{T} \pi_{n(x)} \nabla \varphi_{\eta}(\varepsilon x) d \mathscr{H}^{d-1}(x) \\
& \hat{Q}_{i, 4}:=\varepsilon^{d-2} \sum_{j=1}^{d} \int_{\Gamma_{\eta}}\left[\int_{\mathbf{R}^{d}} c_{i, j}(x, x+\cdot) \sigma(x+\cdot)\right]^{T} \pi_{n(x)} \nabla n_{j}(x) \varphi_{\eta}(\varepsilon x) d \mathscr{H}^{d-1}(x) .
\end{aligned}
$$

Le us consider $\hat{Q}_{i, 1}$. By definition of $\zeta$, the term into brackets is equal to 1 . Therefore,

$$
\hat{Q}_{i, 1}=Q_{i}
$$

We have to establish that the three remaining terms go to 0 as $\varepsilon \downarrow 0$.

Let us fix a point $x \in \Gamma_{\eta}$. The harmonic function $\psi_{x}$ given by Lemma 2.5.c applied in $x+D_{1-\xi}^{\xi}(n(x))$ satisfies $\nabla \psi_{x}(x)=0$ and $n(x) \cdot \nabla \psi_{x} \equiv 0$. In particular, we can substitute $\left[\sigma(x+\cdot)-n(x)-\nabla \psi_{x}(x+\cdot)\right]$ for $[\sigma(x+\cdot)-n(x)]$ in the definition of $\hat{Q}_{i, 2}$. Using (2.13) and the Cauchy-Schwarz inequality, we compute

$$
\begin{array}{r}
\left|\hat{Q}_{i, 2}\right| \leq C(\xi)\|\varphi\|_{\infty} \varepsilon^{d-2} \int_{\Gamma_{\eta}} \sqrt{\mathscr{E}\left(B_{\sqrt{2}}(x)\right)}|h|(x) d \mathscr{H}^{d-1}(x) \\
\leq C(\xi)\|\varphi\|_{\infty} \varepsilon \sqrt{\varepsilon^{d-3 \mathscr{W}(\Sigma)}} \sqrt{\varepsilon^{d-3} \int_{\Gamma_{\eta}} \mathscr{E}\left(B_{\sqrt{2}}(x)\right) d \mathscr{H}^{d-1}(x)} .
\end{array}
$$

Using Fubini and taking into account Lemma 2.2, we obtain

$$
\left|\hat{Q}_{i, 2}\right| \leq C(\xi, \eta)\|\varphi\|_{\infty} \varepsilon \sqrt{\varepsilon^{d-3} \mathscr{W}(\Sigma)} \sqrt{\varepsilon^{d-3} \int_{\Omega} f_{0}(\sigma, \nabla t)} .
$$


Unscaling, we get

$$
\left|\hat{Q}_{i, 2}\right| \leq C(\xi, \eta)\|\varphi\|_{\infty} \sqrt{\mathscr{W}\left(\Sigma_{\varepsilon}\right)} \sqrt{E_{0}} \varepsilon \stackrel{\varepsilon \downarrow 0}{\longrightarrow} 0 .
$$

Next, we consider $\hat{Q}_{i, 3}$. We first notice that $n(x) \cdot \pi_{n(x)} \equiv 0$ so that we can substitute $[\sigma(x+\cdot)-n(x)]$ for $\sigma(x+\cdot)$ in the integral into brackets. Now, since $n(x) \cdot \nabla \psi_{x} \equiv 0$ and $\zeta(\cdot ; n(x))$ is radially symmetric with respect to the direction $n(x)$, the mean value property yields

$$
\int_{\mathbf{R}^{d}} \zeta(y ; n(x)) \nabla \psi_{x}(x+y) d y=\nabla \psi_{x}(x)=0 .
$$

Therefore, we can substitute $\left[\sigma(x+\cdot)-n(x)-\nabla \psi_{x}(x+\cdot)\right]$ for $\sigma(x+\cdot)$ in the definition of $\hat{Q}_{i, 3}$. Proceeding as above, we obtain

$$
\begin{aligned}
& \left|\hat{Q}_{i, 3}\right| \leq C(\xi) \sqrt{\varepsilon^{d-3} \int_{\Omega} f_{0}(\sigma, \nabla t)} \sqrt{\varepsilon^{d-1} \int_{\Sigma}\left|\nabla \varphi_{\eta}\right|^{2} d \mathscr{H}^{d-1}} \varepsilon \\
& \leq C_{\not{q}}(\xi)\left(\left\|\nabla \varphi_{\eta}\right\|_{L^{\infty}\left(\Sigma \cap \mathscr{G}_{\eta}^{\prime \prime}\right)}+\left\|\nabla \varphi_{\eta}\right\|_{\infty} \sqrt{\varepsilon^{d-1} \mathscr{H}^{d-1}\left(\Sigma \cap \mathscr{U}_{\eta}^{\prime \prime}\right)}\right) \varepsilon \\
& \stackrel{(2.23)}{\leq} C_{\mathscr{\varepsilon}}(\xi)\left(\left\|\nabla \varphi_{\eta}\right\|_{L^{\infty}\left(\Sigma \cap G_{\eta}^{\prime \prime}\right)}+\left\|\nabla \varphi_{\eta}\right\|_{\infty} \varepsilon\right) \varepsilon \\
& \stackrel{\text { (4.6)(4.7) }}{\leq} C_{\not \mathcal{Q}}(\xi)\|\varphi\|_{W^{1, \infty}} \varepsilon \stackrel{\varepsilon \downarrow 0}{\longrightarrow} 0 \text {. }
\end{aligned}
$$

For the last term, we can obviously substitute $[\sigma(x+\cdot)-n(x)]$ for $\sigma(x+\cdot)$ but we can still also substitute $\left[\sigma(x+\cdot)-n(x)-\nabla \psi_{x}(x+\cdot)\right]$ as in fact

$$
\begin{aligned}
\int_{\mathbf{R}^{d}} \sum_{j=1}^{d} c_{i, j}(x, x+y) \nabla \psi_{x}^{T}(x+y) \pi_{n(x)} \nabla n_{j}(x) d y \\
\left.=\int_{\mathbf{R}^{d}} \nabla \psi_{x}(x+y) \cdot \pi_{n(x)} c_{i}(x, x+y)\right) d y=0 .
\end{aligned}
$$

To see this, we have to go back to the definition (4.10) of $c_{i}$. Without loss of generality, we assume that $x=0$ and $n(x)=e_{d}$. By (4.10), we have for $1 \leq i \leq$ $d-1$,

$$
c_{i}(0, z)=\zeta\left(z ; e_{d}\right) \nabla n_{i}(0) \quad \stackrel{(4.13)}{\Longrightarrow} \quad \int_{\mathbf{R}^{d}} \nabla \psi_{0}(z) \cdot \pi_{e_{d}} c_{i}(0, z)=0 .
$$

For $i=d$, we have

$$
c_{d}(0, z)=\left.\sum_{k=1}^{d-1} \frac{\partial[\zeta(z ; \bar{n})]}{\partial \bar{n}_{k}}\right|_{\bar{n}=e_{d}} \nabla n_{k}(0) .
$$

Writing $z=\left(z^{\prime}, z_{d}\right)$ and taking into account the relations

$\zeta\left(\left(z^{\prime}, z_{d}\right) ; e_{d}\right)=\zeta\left(\left(z^{\prime},-z_{d}\right) ; e_{d}\right), \quad \zeta(R z ; R \bar{n})=\zeta(z ; \bar{n}) \quad$ for every $R \in \mathrm{SO}(d)$, 
we have using rotations in the plane $\left(e_{k}, e_{d}\right)$,

$$
\left.\frac{\partial \zeta}{\partial \bar{n}_{k}}\left(\left(z^{\prime}, z_{d}\right) ; \bar{n}\right)\right|_{\bar{n}=e_{d}}+\left.\frac{\partial \zeta}{\partial \bar{n}_{k}}\left(\left(z^{\prime},-z_{d}\right) ; \bar{n}\right)\right|_{\bar{n}=e_{d}}=0, \quad \text { for } k=1, \cdots, d-1 .
$$

Hence, since $\nabla \psi_{0}\left(z^{\prime}, z_{d}\right)$ does not depend on $z_{d}$, we have

$$
\int_{\mathbf{R}^{d}} \nabla \psi_{0}(z) \cdot \pi_{e_{d}} c_{d}(0, z)=0 .
$$

Therefore, (4.15) holds.

Substituting $\left[\sigma(x+\cdot)-n(x)-\nabla \psi_{x}(x+\cdot)\right]$ for $\sigma(x+\cdot)$ in the definition of $\hat{Q}_{i, 4}$ and using (2.11) to bound $|\nabla n|(x)=|I I|(x)$ and (2.13) as above, we deduce

$$
\begin{gathered}
\left|\hat{Q}_{i, 4}\right| \leq C(\xi)\|\varphi\|_{\infty} \varepsilon^{d-2} \int_{\Gamma_{\eta}}\left[\mathscr{E}\left(B_{\sqrt{2}}(x)\right)\right]^{3 / 4} d \mathscr{H}^{d-1}(x) \\
\stackrel{\text { (Hölder) }}{\leq} C(\xi)\|\varphi\|_{\infty}\left[\varepsilon^{d-3} \int_{\Gamma_{\eta}} \mathscr{E}\left(B_{\sqrt{2}}(x)\right) d \mathscr{H}^{d-1}(x)\right]^{3 / 4}\left[\varepsilon^{d-1} \mathscr{H}^{d-1}(\Sigma)\right]^{1 / 4} \sqrt{\varepsilon} \\
=C_{\not{q}}(\xi)\left\|\varphi_{\eta}\right\|_{\infty} \sqrt{\varepsilon} \stackrel{\varepsilon \downarrow 0}{\longrightarrow} 0 . \quad(4.16)
\end{gathered}
$$

Proposition 4.1.b then follows from (4.11),(4.12),(4.14) and (4.16).

4.2 Passage to the limit $\varepsilon \downarrow 0$, computation of the lower bound

Proceeding in the same way as to obtain the lower semicontinuity of $\mathscr{W}$ (see (D.8)), we deduce from Proposition 4.1 that

$$
\mathscr{W}(\mathscr{V} 0)=\int_{\Sigma_{0}}\left(\theta_{0}^{+}+\theta_{0}^{-}\right)\left|H_{0}\right|^{2} d \mathscr{H}^{d-1} \leq \liminf _{\varepsilon \downarrow 0} \int_{\Sigma_{\varepsilon}}\left|\hat{h}_{\mathcal{\varepsilon}}\right|^{2} d \mathscr{H}^{d-1} .
$$

By definition of $\hat{h}_{\mathcal{E}}$, we can replace the domain of integration by $\Gamma_{\varepsilon, \eta}:=\varepsilon \Gamma_{(\varepsilon), \eta}$ in the last integral.

Let us recall the definition of the local energy at some point $x \in \Gamma_{(\varepsilon), \eta}$,

$$
\mathscr{E}_{(\varepsilon)}(x):=\int_{B_{3}(x) \cap \Omega_{\varepsilon}}\left[f_{0}\left(\sigma_{(\varepsilon)}, \nabla t_{(\varepsilon)}\right)+\left|\nabla u_{(\varepsilon)}-\nabla t_{(\varepsilon)}\right|^{2}\right] .
$$

We introduce another local energy. Let $\alpha>0$ be a small parameter, we set,

$$
\mathscr{F}_{(\varepsilon), \alpha}(x):=\int_{x+D_{1-2 \xi}^{2 \xi}\left(n_{(\varepsilon)}(x)\right)} f\left(\sigma_{(\varepsilon)}, \nabla t_{(\varepsilon)}\right)+\alpha \mathscr{E}_{(\varepsilon)}(x) .
$$

Remark 4.2 We add the term $\alpha \mathscr{E}_{(\varepsilon)}$ for compactness reasons. This terms controls oscillations of $\nabla u$ and thus of $\Sigma^{0}$.

We then consider local optimization problems associated with this local energy. By frame invariance, we only have to consider the case $x=0$ and $n(x)=e_{d}$. 
Definition 4.3 Let $\zeta, \xi=\xi(\zeta), \alpha>0$ and $\eta \in(0, \xi / 2)$ as above. We set

$$
c_{\zeta, \eta, \alpha}(f):=\frac{1}{\mathscr{H}^{d-1}\left(D_{1}^{\prime}\right)} \inf \left\{\frac{\mathscr{F}_{\alpha}^{\#}\left(a^{\#}\right)}{\left|\hat{h}^{\#}\right|^{2}\left(a^{\#}\right)}: a^{\#} \in \mathscr{S}_{\eta}^{\#}, \hat{h}^{\#}\left(a^{\#}\right) \neq 0\right\} \text {, }
$$

where:

(i) $\mathscr{S}_{\eta}^{\#}$ is the set of quadruplets $a^{\#}=\left(\sigma^{\#}, t^{\#}, u^{\#}, \Sigma^{\#}\right)$ with:

$$
\sigma^{\#} \in L^{2}\left(B_{3}, \mathbf{R}^{d}\right), \quad t^{\#}, u^{\#} \in W^{1,2}\left(B_{3},[-1,1]\right), \quad \Gamma^{\#} \subset D_{1}^{1 / 2}\left(e_{d}\right),
$$

such that:

- $t^{\#}$ is $\omega$-continuous, $u^{\#}$ is $\omega_{\mathscr{\varepsilon}}^{\star}$-continuous, $u^{\#}=t^{\#}$ in $\left\{\left|t^{\#}\right| \geq 9 / 10\right\}$,

- the vector field $\sigma^{\#}$ is divergence free in $\mathscr{D}^{\prime}\left(\left[\left|t^{\#}\right|<1\right]\right)$,

- $u^{\#}$ is harmonic and $\left|\nabla u^{\#}\right| \geq 1 / 2$ in $D_{1}^{1 / 2}\left(e_{d}\right)$,

- $\Gamma^{\#}$ is the hypersurface:

$$
\Gamma^{\#}=\left[u^{\#}=0\right] \cap D_{1}^{1 / 2}\left(e_{d}\right),
$$

and moreover, $0 \in \Gamma^{\#}$ and $\nabla u^{\#} /\left|\nabla u^{\#}\right|(0)=e_{d}$,

- we have the energy bound (see (4.4))

$$
\int_{B_{\lambda} \cap \Omega^{\#}}\left(f_{0}\left(\sigma^{\#}, \nabla t_{\varepsilon}\right)+\left|\nabla u^{\#}-\nabla t^{\#}\right|^{2}\right) \leq \beta_{\eta} .
$$

(ii) The local energy $\mathscr{F}_{\alpha}^{\#}\left(a^{\#}\right)$ is modeled on $\mathscr{F}_{(\varepsilon), \alpha}$ :

$$
\mathscr{F}_{\alpha}^{\#}\left(a^{\#}\right):=\int_{D_{1-2 \xi}^{2 \xi}} f\left(\sigma^{\#}, \nabla t^{\#}\right)+\alpha \mathscr{E}^{\#}\left(a^{\#}\right),
$$

with

$$
\mathscr{E}^{\#}\left(a^{\#}\right):=\int_{B_{3} \cap\left[t^{\#} \mid<1\right]}\left[f_{0}\left(\sigma^{\#}, \nabla t^{\#}\right)+\left|\nabla u^{\#}-\nabla t^{\#}\right|^{2}\right] .
$$

(iii) The approximate curvature $\hat{h}^{\#}\left(a^{\#}\right)$ is defined as (compare to (4.5)):

$$
\hat{h}^{\#}\left(a^{\#}\right):=\int_{\mathbf{R}^{d}} \sigma^{\#} \cdot\left(\zeta_{\perp} \nabla_{y} \zeta_{/ /}\right)\left(y ; e_{d}\right) d y .
$$

The parameter $\eta \in(0, \xi / 2)$ being fixed, according to the above definition we have for every $x \in \Gamma_{(\varepsilon), \eta}, \varepsilon \in(0,1]$ :

$$
\left|\hat{h}_{(\varepsilon)}\right|^{2}(x) \leq \frac{1}{c_{\zeta, \eta, \alpha}(f)} \frac{1}{\mathscr{H}^{d-1}\left(D_{1}^{\prime}\right)} \mathscr{F}_{(\varepsilon), \alpha}(x) .
$$

Using this inequality in (4.17), we obtain

$$
\begin{aligned}
& c_{\zeta, \eta, \alpha}(f) \mathscr{W}\left(\mathscr{V}_{0}\right) \\
& \quad \leq \frac{1}{\mathscr{H}^{d-1}\left(D_{1}^{\prime}\right)} \liminf _{\varepsilon \downarrow 0} \varepsilon^{d-3} \int_{\Gamma_{(\varepsilon), \eta}} \mathscr{F}_{(\varepsilon), \alpha}(x) d \mathscr{H}^{d-1}(x) .
\end{aligned}
$$


Let us consider the integral in the right hand side. Replacing $\mathscr{F}_{(\varepsilon), \alpha}(x)$ by its definition, applying Fubini and taking into account Lemma 2.4, we get

$$
\begin{aligned}
\int_{\Gamma_{(\varepsilon), \eta}} \mathscr{F}_{(\varepsilon), \alpha}(x) d \mathscr{H}^{d-1}(x) & \leq \int_{\Omega_{(\varepsilon)}^{\xi / 2}} f\left(\sigma_{(\varepsilon)}, \nabla t_{(\varepsilon)}\right) q_{(\varepsilon)} \\
& +\alpha \int_{\Omega_{(\varepsilon)}}\left[f_{0}\left(\sigma_{(\varepsilon)}, \nabla t_{(\varepsilon)}\right)+\left|\nabla u_{(\varepsilon)}-\nabla t_{(\varepsilon)}\right|^{2}\right] q_{(\varepsilon)}^{\prime} .
\end{aligned}
$$

with $\Omega_{(\varepsilon)}^{\xi / 2}=\left[\left|t_{(\varepsilon)}\right|(y)<1-\xi / 2\right]$,

$$
q_{(\varepsilon)}(y):=\mathscr{H}^{d-1}\left(\left\{x \in \Gamma_{(\varepsilon), \eta}: y \in x+D_{1-2 \xi}^{2 \xi}(n(x))\right\}\right) .
$$

and $q_{(\varepsilon)}^{\prime}(y)=\mathscr{H}^{d-1}\left(\Sigma_{(\varepsilon)}^{0} \cap B_{3}(y)\right)$.

By inequality (2.14) of Lemma 2.6, we have $q_{(\varepsilon)}(y) \leq \mathscr{H}^{d-1}\left(D_{1}^{\prime}\right)(1+s(\eta))$ with $s(\eta) \rightarrow 0$ as $\eta \downarrow 0$. On the other hand, thanks to (2.22), we have $q_{(\varepsilon)}^{\prime} \leq C_{\not \notin}$. Hence,

$$
\begin{aligned}
\frac{1}{\mathscr{H}^{d-1}\left(D_{1}^{\prime}\right)} \int_{\Gamma_{(\varepsilon), \eta}} \mathscr{F}_{(\varepsilon), \alpha}(x) d \mathscr{H}^{d-1}(x) & \\
\leq & \leq(1+s(\eta)) \mathscr{F}\left(\sigma_{(\varepsilon)}, \nabla t_{(\varepsilon)}, \Omega_{(\varepsilon)}\right)+C_{\not \varepsilon} \alpha .
\end{aligned}
$$

Putting this estimate in (4.20), unscaling and sending $\eta$ to 0 and then $\alpha$ to 0 , we end with

$$
\left[\liminf _{\alpha \downarrow 0} \liminf _{\eta \downarrow 0} c_{\zeta, \eta, \alpha}(f)\right] \mathscr{W}\left(\mathscr{V}_{0}\right) \leq \liminf _{\varepsilon \downarrow 0} \frac{\mathscr{F}\left(\sigma_{\varepsilon}, \nabla t_{\varepsilon}, \Omega_{\varepsilon}\right)}{\varepsilon^{3}} .
$$

Theorem 1.2 then follows from the next lemma which states the required lower bound for the local optimization problems.

\section{Lemma 4.1 (Local optimization)}

(a) For every $\alpha>0$, there holds:

$$
\liminf _{\eta \downarrow 0} c_{\zeta, \eta, \alpha}(f) \geq c_{0, \zeta}(f):=\frac{\operatorname{det} L}{2 L_{2,2}}\left(\mathscr{H}^{d-1}\left(D_{1}^{\prime}\right) \int_{D_{1}^{1}}\left|\partial_{y_{d}} \zeta\right|^{2}\left(y ; e_{d}\right) d y\right)^{-1}
$$

where $L$ is the $2 \times 2$ matrix defined in Lemma 3.1.

(b) There exists a sequence of admissible functions $\left(\zeta_{k}\right) \subset W^{1, \infty}\left(\mathbf{R}^{d} \times S^{d-1}, \mathbf{R}_{+}\right)$ (i.e. $\zeta_{k}$ complies to the constraints of Definition 4.1) such that

$$
c_{0, \zeta_{k}}(f) \stackrel{k \uparrow \infty}{\longrightarrow} c_{0}(f)=\frac{\operatorname{det} L}{3 L_{2,2}}
$$

Proof Let us fix $\alpha>0$ and let $\zeta$ be as in Definition 4.1. We first establish $(a)$. For this, we consider a minimizing sequence $a_{k}^{\#}=\left(\sigma_{k}^{\#}, \nabla t_{k}^{\#}, \nabla u_{k}^{\#}, \Sigma_{k}^{\#}\right) \subset \mathscr{S}_{\eta_{k}}^{\#}$ such that $\eta_{k} \downarrow 0, \hat{h}_{\zeta}^{\#}\left(a_{k}^{\#}\right) \neq 0$ and

$$
\lim _{k \uparrow \infty} \frac{\mathscr{F}_{\alpha}^{\#}\left(a_{k}^{\#}\right)}{\left|\hat{h}^{\#}\right|^{2}\left(a_{k}^{\#}\right)}=\mathscr{H}^{d-1}\left(D_{1}^{\prime}\right) \liminf _{\eta \downarrow 0} c_{\zeta, \eta, \alpha}(f) .
$$


Using the reflection symmetry with respect to the hyperplane $e_{d}^{\perp}$, we assume without loss of generality that $\hat{h}_{\zeta}^{\#}\left(a_{k}^{\#}\right)>0$.

We start by proving that $c_{\zeta, \eta, \alpha}(f)$ is uniformly bounded with respect to $\eta$. Lemmas 2.4 and 2.5 have been stated for the quadruplets

$$
\left(\sigma_{(\varepsilon)}, t_{(\varepsilon)}, u_{(\varepsilon)}, \Sigma_{(\varepsilon)}^{0}\right)
$$

but by definition of the sets $\mathscr{S}_{\eta}^{\#}$ they obviously also apply to the elements of the sequence $\left(a_{k}^{\#}\right)$ at point $x=0$. In particular, $\sigma_{k}^{\#} \rightarrow e_{d}$ in $L^{2}\left(D_{1-\xi}^{\xi}\right)$, which implies $\hat{h}_{\zeta}^{\#}\left(a_{k}^{\#}\right) \rightarrow 0$.

Now let $\hbar \in(0,1]$ and let us define $a^{\#}:=\left(\sigma^{\#}, t^{\#}, u^{\#}, \Sigma^{\#}\right)$, as

$$
\sigma^{\#}(y)=e_{d}+\hbar\left(\frac{1}{d-1} y^{\prime}-y_{d} e_{d}\right), \quad t^{\#}(y)=u^{\#}(y)=y_{d}, \quad \Sigma^{\#}=D_{1}^{\prime} .
$$

We easily check that $\hat{h}_{\zeta}^{\#}\left(a^{\#}\right)=\hbar$, that $a^{\#} \in \mathscr{S}_{\eta}^{\#}$ for $\eta \in\left(0, \eta_{0}\right)$ for some $\eta_{0}>0$ depending on $\hbar$ and that

$$
\mathscr{F}_{\alpha}^{\#}\left(a^{\#}\right) \leq C(f) \hbar^{2}=C(f)\left|\hat{h}^{\#}\left(a^{\#}\right)\right|^{2} .
$$

Therefore, $c_{\zeta, \eta, \alpha}(f) \leq C(f) / \mathscr{H}^{d-1}\left(S^{d-1}\right)$. Since $\left(a_{k}^{\#}\right)$ is a minimizing sequence, we can restrict to $\mathscr{F}_{\alpha}^{\#}\left(a_{k}^{\#}\right) \leq C(f)\left|\hat{h}^{\#}\left(a_{k}^{\#}\right)\right|^{2}$.

Using the notation $\hbar_{k}:=\hat{h}^{\#}\left(a_{k}^{\#}\right)$, we now assume

$$
\mathscr{F}_{\alpha}^{\#}\left(a_{k}^{\#}\right) \leq C(f)\left|\hbar_{k}\right|^{2} \stackrel{k \uparrow \infty}{\longrightarrow} 0 .
$$

Let us call $\psi_{k}^{\#}$ the harmonic function given by Lemma 2.5.c applied to $a_{k}^{\#}$ and let us set $\psi_{k}^{b}:=\left(1 / \sqrt{\hbar_{k}}\right) \psi_{k}^{\#}$. We define a vector field $\sigma_{k}^{b}$ and a function $t_{k}^{b}$ on $D_{1-2 \xi}^{2 \xi}$ by

$$
\sigma_{k}^{\#}=e_{d}+\sqrt{\hbar_{k}} \nabla \psi_{k}^{b}+\hbar_{k} \sigma_{k}^{b}, \quad \nabla t_{k}^{\#}=e_{d}+\sqrt{\hbar_{k}} \nabla \psi_{k}^{b}+\hbar_{k} \nabla t_{k}^{b}
$$

(To fix the the constant, we assume $\int_{D_{1-2 \xi}^{2 \xi}} t_{k}^{b}=0$ ) Using (4.21) in the estimates of Lemma 2.5.c, we have

$$
\left\|\nabla \psi_{k}^{b}\right\|_{L^{2}\left(D_{1-3 \xi / 2}^{0}\right)}^{2}+\left\|\sigma_{k}^{b}\right\|_{L^{2}\left(D_{1-2 \xi}^{2 \xi}\right)}^{2}+\left\|\nabla t_{k}^{b}\right\|_{L^{2}\left(D_{1-2 \xi}^{2 \xi}\right)}^{2} \leq C(f, \alpha, \xi) .
$$

Hence, up to extraction of a subsequence, there exist two functions

$$
\psi_{\infty}^{b}, t_{\infty}^{b} \in W^{1,2}\left(D_{1-2 \xi}^{2 \xi}\right)
$$


and a vector field $\sigma_{\infty}^{b} \in L^{2}\left(D_{1-2 \xi}^{2 \xi}, \mathbf{R}^{d}\right)$ such that $\psi_{\infty}^{b}$ is harmonic, $\partial_{d} \psi_{\infty}^{b} \equiv 0, \sigma_{\infty}^{b}$ is divergence free and

$$
\begin{array}{r}
\nabla \psi_{k}^{b} \stackrel{k \uparrow \infty}{\longrightarrow} \nabla \psi_{\infty}^{b} \quad \text { in } C^{\infty}\left(\overline{D_{1-2 \xi}^{0}}\right), \\
\sigma_{k}^{b} \stackrel{k \uparrow \infty}{\longrightarrow} \sigma_{\infty}^{b}, \quad \nabla t_{k}^{b} \stackrel{k \uparrow \infty}{\longrightarrow} \nabla t_{\infty}^{b} \quad \text { weakly in } L^{2}\left(D_{1-2 \xi}^{2 \xi}\right) .
\end{array}
$$

To study the asymptotic behavior of $\mathscr{F}_{\zeta}^{\#}\left(a_{k}^{\#}\right)$, we are going to use (4.22) and a Taylor expansion of $f$. The latter requires point-wise bounds. First, by (4.24), there exists a constant $K>0$ such that

$$
\sup _{D_{1-2 \xi}^{0}}\left|\nabla \psi_{k}^{b}\right| \leq K .
$$

We do not have pointwise bounds on the sequence $\left(\sigma_{k}^{b}, \nabla t_{k}^{b}\right)$ and we are led to reduce the domains of definition of these functions.

For this, let us introduce a small parameter $\rho>0$. By the preceding estimate, for $k$ large enough, say $k \geq k_{0}(\rho)$, there holds

$$
\sqrt{\hbar_{k}}\left|\nabla \psi_{k}^{b}\right|<\rho \quad \text { on } D_{1-2 \xi}^{0} .
$$

Now, for $k \geq 0$, we set

$$
S_{\rho, k}:=\left\{y \in D_{1-2 \xi}^{2 \xi}:\left|\left(\sigma_{k}^{b}(y), \nabla t_{k}^{b}(y)\right)\right|<\rho /\left|\hbar_{k}\right|\right\} .
$$

Notice, for later use, that since $\left(\sigma_{k}^{b}\right)$ and $\left(\nabla t_{k}^{b}\right)$ are bounded in $L^{2}\left(D_{1-2 \xi}^{2 \xi}\right)$ and $\hbar_{k} \rightarrow 0$, we have

$$
\mathscr{H}^{d-1}\left(D_{1-2 \xi}^{2 \xi} \backslash S_{\rho, k}\right) \stackrel{k \uparrow \infty}{\longrightarrow} 0 .
$$

Since $f$ is of class $C^{2}$ in some neighborhood of $\mathbb{S}^{d-1}$, there exists $\rho_{0}>0$ and a sublinear modulus of continuity $\omega_{f}(r) \stackrel{r \downarrow 0}{\rightarrow} 0$ such that for $\sigma^{\prime}, \tau^{\prime}, \sigma^{\prime \prime}, \tau^{\prime \prime} \in B_{\rho_{0}}\left(e_{d}\right)$, we have

$$
\left|D^{2} f\left(\sigma^{\prime \prime}, \tau^{\prime \prime}\right)-D^{2} f\left(\sigma^{\prime}, \tau^{\prime}\right)\right| \leq \omega_{f}\left(\left|\left(\sigma^{\prime \prime}-\sigma^{\prime}, \tau^{\prime \prime}-\tau^{\prime}\right)\right|\right) .
$$

Let us now assume $\rho \in\left(0, \rho_{0} / 2\right)$ and let us fix for a moment $k \geq k_{0}(\rho)$ and $y \in S_{k}$ so that by construction, we have $\sigma_{k}^{\#}(y), \nabla t_{k}^{\#}(y) \in B_{\rho_{0}}\left(e_{d}\right)$. We set

$$
m:=e_{d}+\sqrt{\hbar_{k}} \nabla \psi_{k}^{b}(y), \quad n:=m /|m|,
$$

(we also have $n \in B_{\rho_{0}}\left(e_{d}\right)$ ). Let us also introduce the pairs of vectors,

$$
M:=(m, m), \quad N:=(n, n), \quad \text { and } \quad Q:=\hbar_{k}\left(\sigma_{k}^{b}(y), \nabla t_{k}^{b}(y)\right),
$$

We estimate $f\left(\sigma_{k}^{\#}(y), \nabla t_{k}^{\#}(y)\right)$ from below by using (4.22) and a Taylor expansion of $f$ at $N$. Taking into account $f(N)=0, D f(N)=0$ and (4.27), we obtain

$$
\begin{aligned}
f\left(\sigma_{k}^{\#}(y),\right. & \left.\nabla t_{k}^{\#}(y)\right)=f(N+(M-N+Q)) \\
& \geq \frac{1}{2}(M-N+Q)^{T} D^{2} f(N)(M-N+Q)-\frac{1}{2} \omega_{f}(2 \rho)|M-N+Q|^{2} .
\end{aligned}
$$


We rewrite the quadratic term as

$$
D^{2} f(N)=D^{2}\left(f\left(e_{d}, e_{d}\right)\right)+\left[D^{2} f(N)-D^{2}\left(f\left(e_{d}, e_{d}\right)\right] .\right.
$$

Since, $\left|n-e_{d}\right|<\rho$, this leads to

$$
\begin{aligned}
& f\left(\sigma_{k}^{\#}(y), \nabla t_{k}^{\#}(y)\right) \\
& \geq \frac{1}{2}(M-N+Q)^{T} D^{2} f\left(e_{d}, e_{d}\right)(M-N+Q)-\omega_{f}(2 \rho)|M-N+Q|^{2} .
\end{aligned}
$$

Now performing a Taylor expansion of $m-n$, we get

$$
m-n=\frac{\hbar_{k}}{2}\left|\nabla \psi_{k}^{b}\right|^{2}(y) e_{d}+O\left(\left|\hbar_{k}\right|^{3 / 2} K^{3}\right) .
$$

Hence, setting

$$
v_{k}^{b}:=\sigma_{k}^{b}+\frac{\left|\nabla \psi_{k}^{b}\right|^{2}}{2} e_{d}, \quad \tau_{k}^{b}:=\nabla t_{k}^{b}+\frac{\left|\nabla \psi_{k}^{b}\right|^{2}}{2} e_{d},
$$

we obtain,

$$
M-N+Q=\hbar_{k}\left(v_{k}^{b}, \tau_{k}^{b}\right)+O\left(\left|\hbar_{k}\right|^{3 / 2} K^{3}\right) .
$$

Substituting this expansion in (4.28), dividing by $\left(\hbar_{k}\right)^{2}$ and integrating on $S_{\rho, k}$, we get

$$
\begin{aligned}
\frac{1}{\left|\hbar_{k}\right|^{2}} \mathscr{F}_{\zeta}^{\#}\left(a_{k}^{\#}\right) \geq \frac{1}{2} \int_{S_{\rho, k}}\left(v_{k}^{b}(y), \tau_{k}^{b}(y)\right)^{T} D^{2} f\left(e_{d}, e_{d}\right)\left(v_{k}^{b}(y), \tau_{k}^{b}(y)\right) \\
-2 \omega_{f}(2 \rho)\left(2 C(K) \mathscr{H}^{d-1}\left(D_{1}^{\prime}\right)+C(\alpha, \xi)\right),
\end{aligned}
$$

where we have used (4.23) to bound the remainder.

Let $\chi_{\rho, k}$ be the characteristic function of $S_{\rho, k}$. We deduce from (4.24) (4.25) and (4.26), that

$$
\begin{array}{r}
\left(\chi_{\rho, k} v_{k}^{b}, \chi_{\rho, k} \tau_{k}^{b}\right) \stackrel{k \uparrow \infty}{\longrightarrow}\left(v_{\infty}^{b}, \tau_{\infty}^{b}\right):=\left(\sigma_{\infty}^{b}+\frac{\left|\nabla \psi_{\infty}^{b}\right|^{2}}{2} e_{d}, \nabla t_{\infty}^{b}+\frac{\left|\nabla \psi_{\infty}^{b}\right|^{2}}{2} e_{d}\right) \\
\text { weakly in } L^{2}\left(D_{1-2 \xi}^{2 \xi}\right) .
\end{array}
$$

By positivity of $D^{2} f\left(e_{d}, e_{d}\right)$, the functional

$$
W \in L^{2}\left(D_{1-2 \xi}^{2 \xi}\right) \mapsto \int_{O} W^{T} D^{2} f\left(e_{d}, e_{d}\right) W
$$

is lower semicontinuous with respect to the topology of weak convergence in $L^{2}$. Therefore, sending $k$ to $+\infty$ in (4.29), then sending $\eta$ to 0 and taking into account the fact that $\rho>0$ is arbitrary, we end with

$$
\mathscr{H}^{d-1}\left(D_{1}^{\prime}\right)\left[\liminf _{\eta \downarrow 0} c_{\zeta, \eta, \alpha}\right] \geq \frac{1}{2} \int_{D_{1-2 \xi}^{2 \xi}}\left(v_{\infty}^{b}, \tau_{\infty}^{b}\right)^{T} D^{2} f\left(e_{d}, e_{d}\right)\left(v_{\infty}^{b}, \tau_{\infty}^{b}\right) .
$$


We simplify further the right hand side by using the structure of $D^{2} f\left(e_{d}, e_{d}\right)$ described in Lemma 3.1. Calling $p=\left(v_{\infty, d}^{b}, \tau_{\infty, d}^{b}\right)$ the last components of the vector fields $v_{\infty}^{b}, \tau_{\infty}^{b}$, we obtain,

$$
\mathscr{H}^{d-1}\left(D_{1}^{\prime}\right)\left[\liminf _{\eta \downarrow 0} c_{\zeta, \eta, \alpha}\right] \geq \frac{1}{2} \int_{D_{1-2 \xi}^{2 \xi}} p^{T} L p .
$$

where the positive symmetric $2 \times 2$ matrix $L$ is defined by (3.1).

Now we pass to the limit in the affine constraint. Putting the expansion (4.22) of $\sigma_{k}^{\#}$ in the definition of $\hbar_{k}=h_{\zeta}^{\#}\left(a_{k}^{\#}\right)$ and using the facts that $\psi_{k}^{\#}$ is harmonic and does not depend on $y_{d}$, we deduce from the constraint (4.18),

$$
\int_{\mathbf{R}^{d}} \sigma_{k}^{b} \cdot\left(\zeta_{\perp} \nabla_{y} \zeta_{/ /}\right)\left(y ; e_{d}\right) d y=1 .
$$

Passing to the limit $k \uparrow \infty, \sigma_{\infty}^{b}$ satisfies the constraint

$$
\int_{\mathbf{R}^{d}} \sigma_{\infty}^{b} \cdot\left(\zeta_{\perp} \nabla_{y} \zeta_{/ /}\right)\left(y ; e_{d}\right) d y=1 .
$$

Since $\nabla \cdot \sigma_{\infty}^{b} \equiv 0$ and $\zeta$ is compactly supported, this amounts to

$$
\int_{\mathbf{R}^{d}} \sigma_{\infty, d}^{b}(y) \partial_{y_{d}} \zeta\left(y ; e_{d}\right) d y=-1
$$

Now, since $p_{1}=\sigma_{\infty, d}^{b}+\left|\nabla \psi_{\infty}^{b}\right|^{2} / 2$ and $\nabla \psi_{\infty}^{b}$ does not depend on $y_{d}$, we also have

$$
\int_{\mathbf{R}^{d}} p_{1}(y) \partial_{y_{d}} \zeta\left(y ; e_{d}\right) d y=-1 .
$$

Eventually, we have to optimize the right hand side of (4.30) with respect to the elements $p \in L^{2}\left(D_{1-2 \xi}^{\xi}, \mathbf{R}^{2}\right)$ satisfying the constraint (4.32). Since $L$ is positive definite, the minimizer exists and is unique. The Euler-Lagrange equations lead to $p_{o p t}=\lambda \partial_{y_{d}} \zeta\left(\cdot ; e_{d}\right) L^{-1}\left(\begin{array}{l}1 \\ 0\end{array}\right)$, where the Lagrange multiplier

$$
\lambda=\frac{\operatorname{det} L}{L_{2,2}\left\|\partial_{y_{d}} \zeta\left(\cdot ; e_{d}\right)\right\|_{L^{2}\left(D_{1}^{0}\right)}^{2}}
$$

is fixed by the constraint. The minimal energy is $\lambda / 2$. Together with (4.30), this leads to

$$
\mathscr{H}^{d-1}\left(D_{1}^{\prime}\right)\left[\liminf _{\eta \downarrow 0} c_{\zeta, \eta, \alpha}\right] \geq \frac{\operatorname{det} L}{2 L_{2,2}\left\|\partial_{y_{d}} \zeta\left(\cdot ; e_{d}\right)\right\|_{L^{2}\left(D_{1}^{0}\right)}^{2}}
$$

and $(a)$ is proved. 
(b) We now optimize in $\zeta$. We are looking for the largest possible lower bound

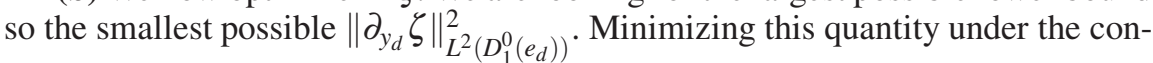
straints $\int_{D_{1}^{0}\left(e_{d}\right)} \zeta=1, \partial_{y_{d}} \zeta \in L^{2}\left(D_{1}^{0}\left(e_{d}\right)\right)$ and $\zeta=0$ on $\left\{y \in \partial D_{1}^{0}\left(e_{d}\right): y_{d}= \pm 1\right\}$, we obtain as unique minimizer

$$
\zeta_{0}(y)=\frac{3}{4 \mathscr{H}^{d-1}\left(D_{1}^{\prime}\right)}\left(1-y_{d}^{2}\right) \quad \text { with } \quad \mathscr{H}^{d-1}\left(D_{1}^{\prime}\right) \int_{D_{1}^{0}\left(e_{d}\right)}\left|\partial_{y_{d}} \zeta_{0}\right|^{2}=\frac{3}{2} .
$$

As required, we have $c_{0, \zeta_{0}}(f)=(\operatorname{det} L) /\left(3 L_{2,2}\right)$ and $\zeta_{\text {opt }}$ is a product of smooth functions depending on tangential and thickness coordinates respectively. However, we can not use $\zeta_{0}$ in Definition 4.1 because it is not compactly supported in $D_{1}^{0}\left(e_{d}\right)$. Anyway, we can approximate $\zeta_{0}$ by a sequence of compactly supported function $\left(\zeta_{j}\right) \subset W^{1, \infty}\left(D_{1}^{0}\left(e_{d}\right) \times S^{d-1}\right)$ that comply to the requirements of Definition 4.1. For example:

$$
\left.\zeta_{j}\left(R\left(y^{\prime}, y_{d}\right) ; R e_{d}\right)\right):=\zeta_{j}\left(\left(y^{\prime}, y_{d}\right) ; e_{d}\right):=\frac{j+1}{j} \frac{\theta_{j}\left(y^{\prime}\right)}{\left\|\theta_{j}\right\|_{L^{1}\left(D_{1}^{\prime}\right)}} \zeta_{0}\left(y^{\prime}, \frac{j+1}{j} y_{d}\right),
$$

with $\theta_{j} \in \mathscr{D}\left(D_{1}^{\prime},[0,1]\right)$ satisfying $\theta_{j}\left(y^{\prime}\right)=1$ for $\left|y^{\prime}\right| \leq 1-2 / j$. For such a sequence, we have $c_{0, \zeta_{j}}(f) \rightarrow(\operatorname{det} L) /\left(3 L_{2,2}\right)$ as $j \uparrow \infty$ which establishes part (b) of the lemma.

\section{Concluding remarks}

Before addressing the case of membranes with non-zero spontaneous curvature, let us discuss the hypotheses of our main results.

\section{Upper-bound in the general case.}

To complete the $\Gamma$-limit analysis, we should extend the construction of a recovery family of Theorem 1.3 to any data $\mathscr{V}_{0} \in \mathscr{A}_{0}(R, S)$. Namely, we should prove that given $\mathscr{V}_{0} \in \mathscr{A}_{0}(R, S)$ there exists a family $\left\{a_{\varepsilon}\right\}_{0<\varepsilon<1}$, with $a_{\varepsilon} \in \mathscr{A}_{\varepsilon}(R, S)$ such that $\mathscr{V}_{\varepsilon}^{\star}\left(a_{\varepsilon}\right) \rightarrow \mathscr{V}_{0}$ and $\mathscr{F}\left(a_{\varepsilon}\right) / \varepsilon^{3} \rightarrow \mathscr{W}\left(\Sigma_{0}\right)$ as $\varepsilon \downarrow 0$. A natural way for this would be to answer positively the following question.

Question 5.1 Let $\mathscr{V}_{0} \in \mathscr{A}_{0}(R, S)$. Does there exist a sequence of smooth open sets $\left(O_{k}\right) \subset B_{R}$ with outward unit normal $v_{k}$ and boundary $\Sigma_{k}$ such that (1.5) holds and furthermore,

$$
\mathscr{W}\left(\Sigma_{k}\right) \stackrel{k \uparrow \infty}{\longrightarrow} \mathscr{W}\left(\mathscr{V}_{0}\right) ?
$$

As described in Section 2.6, the elements of $\mathscr{A}_{0}$ are oriented rectifiable $(d-1)$ varifolds with a generalized mean curvature in $L^{2}$. To our knowledge the best regularity result established for varifolds with a generalized mean curvature is due to A. Menne [10] who established their $C^{2}$-rectifiabilty (see also R. Schätzle [12] and references therein). In this context, giving a positive answer to Question 5.1 seems a difficult task. 
The uniform continuity hypothesis.

We would like to get rid of the uniform continuity hypothesis on $x \mapsto \varepsilon^{-1} t_{\varepsilon}(\varepsilon x)$ (Hypothesis 1) and establish the compactness result under sole energy bounds.

We could try to relax the hypothesis by approximating the functions

$$
t_{\varepsilon} \in W_{l o c}^{1,2}\left(\mathbf{R}^{d},[-\varepsilon, \varepsilon]\right)
$$

by Lipschitz continuous functions $\hat{t}_{\varepsilon}$ as in the Appendix of [6] - see also Evans and Gariepy [5], Section 6.6.3, Theorem 3. In doing this, we should also substitute $\hat{\Omega}_{\varepsilon}=\left[\left|\hat{t}_{\varepsilon}\right|<\varepsilon\right]$ for the domain $\Omega_{\varepsilon}$ and find a vector field $\hat{\sigma}_{\varepsilon}$ which approximates $\sigma_{\varepsilon}$ and is divergence free in $\hat{\Omega}_{\varepsilon}$. The fact that the topologies of $\Omega_{\varepsilon}$ and $\hat{\Omega}_{\varepsilon}$ could be different is an obstacle for such construction. For instance, if $\sigma=x /|x|^{d}$ in $B_{1} \backslash \overline{B_{\rho}}$ then we can not extend $\sigma$ as a divergence free vector field in $B_{1}$. As a conclusion, we leave the relaxation of the uniform continuity hypothesis as an open problem.

\section{The confining hypothesis.}

We would also like to weaken the confining hypothesis: $\left[t_{\varepsilon} \equiv \varepsilon\right]$ in the complement of $B_{R}$. This hypothesis is only needed to prevent $\Sigma_{\varepsilon}$ from escaping to infinity when passing to the limit $\varepsilon \downarrow 0$. This is feasible, at least for $d=2$ and $d=3$.

In dimension $d=2$, the bad sets introduced in the compactness step are empty for $\varepsilon$ small enough: indeed, the number of bad balls in Lemma 2.7 satisfies $N \leq$ $C_{\xi}(\beta) \varepsilon$. Therefore, for $\varepsilon$ small enough $\Sigma_{\varepsilon}=\left[u_{\varepsilon}=0\right]$ is a finite union of nonintersecting analytic Jordan curves. Their total length and their elastic energy $\mathscr{W}\left(\Sigma_{\varepsilon}\right)$ is uniformly bounded. Substituting the condition " $t_{\varepsilon} \equiv \varepsilon$ in the neighborhood of infinity" for the confining assumption, we can track the different connected components by using finitely many translations and obtain at the limit a finite number of $W^{1,2}$-Jordan curves with total mass $S$.

In dimension $d=3$, the number of bad balls is only uniformly bounded: $N \leq C_{\mathscr{q}}(\beta)$. However, the number of connected components of $\Sigma_{\varepsilon}$ is uniformly bounded. Indeed, the Willmore energy of each component is bounded from below by $4 \pi$. Moreover, according to a result of Simon [14], Lemma 1.1 for every connected component $\Sigma_{\varepsilon}^{i}$ we have

$$
\operatorname{diam}\left(\Sigma_{\varepsilon}^{i}\right) \leq C \sqrt{\mathscr{H}^{d-1}\left(\sum_{\varepsilon}^{i}\right) \mathscr{W}\left(\Sigma_{\varepsilon}^{i}\right)} \quad\left(\leq C_{\not d}\right) .
$$

Hence, considering separately each connected component and using translations, we can assume that each connected component satisfies the confining assumption.

5.1 The case of a material with non vanishing spontaneous curvature

The model introduced in [11] is more general than the one we have considered so far. In Sections I.1.3, I.1.4, the divergence free condition is relaxed to

$$
\nabla \cdot\left[\frac{\sigma_{\varepsilon}}{\rho\left(\mu t_{\varepsilon}\right)}\right]=0 \quad \text { in } \mathscr{D}^{\prime}\left(\Omega_{\varepsilon}\right),
$$


where $\rho$ is a smooth positive function satisfying $\rho(0)=1$ and $\rho^{\prime}(0)=-1$ and $\mu \in \mathbf{R}$ is a given value of the spontaneous curvature. As expected in [11], the results of the present paper extend to this case. More precisely, we claim that if we substitute for the Willmore functional, the following energy introduced by Helfrich [8],

$$
\mathscr{W}_{\mu}(\Sigma):=\int_{\Sigma}|h-\mu|^{2} d \mathscr{H}^{d-1},
$$

then Theorems 1.1, 1.2 and 1.3 generalize to this setting without further changes.

Let us describe the modifications to be made in the proofs. We first consider the construction of the recovery family of Theorem 1.3. In this proof, we have to change the definition of $\sigma_{\varepsilon}$ by adding the factor $\rho(\mu t(y))$ in the right hand side of (3.2) so that, by construction, $\nabla \cdot[1 / \rho(\mu t) \sigma] \equiv 0$ in $\Omega_{\varepsilon}$ for $0<\varepsilon<\varepsilon_{\star}^{\prime} / 2$. Taking into account $\rho(0)=1, \rho^{\prime}(0)=-1$, the Taylor expansion (3.4) is changed to

$$
\sigma_{\varepsilon}\left(x+s v_{0}(x)\right)=v_{0}(x)+s\left[h_{0}(x)-\mu\right] v_{0}(x)+O\left(s^{2}\right) .
$$

This amounts to substitute $h_{0}-\mu$ for the mean curvature $h_{0}$. As claimed, we get,

$$
c_{0}(f) \mathscr{W}_{\mu}\left(\Sigma_{0}\right)=\lim _{\varepsilon \downarrow 0} \frac{\mathscr{F}\left(a_{\varepsilon}\right)}{\varepsilon^{3}} .
$$

Let us now consider $R, S>0$ and a family $a_{\varepsilon}=\left(\sigma_{\varepsilon}, \nabla t_{\varepsilon}, \Omega_{\varepsilon}\right)$ satisfying the hypotheses of Theorem 1.1 except that we substitute (5.1) for the divergence free condition on $\sigma_{\varepsilon}$. The (obvious) key idea is to set

$$
\sigma_{\varepsilon}^{\mu}:=\left[1 / \rho\left(\mu t_{\varepsilon}\right)\right] \sigma_{\varepsilon}
$$

and to consider the configurations $a_{\varepsilon}^{\mu}:=\left(\sigma_{\varepsilon}^{\mu}, \nabla t_{\varepsilon}, \Omega_{\varepsilon}\right)$. By construction, we have $a_{\varepsilon}^{\mu} \in \mathscr{A}_{\varepsilon}(R)$. Let us introduce the notation

$$
Q_{\varepsilon}^{\mu}:=\mathscr{Q}\left(\sigma_{\varepsilon}^{\mu}, \nabla t_{\varepsilon}\right) /(2 \varepsilon), \quad F_{\varepsilon}^{\mu}:=\mathscr{F}_{0}\left(a_{\varepsilon}^{\mu}\right) / \varepsilon^{3} .
$$

We claim that $\left\{a_{\varepsilon}^{\mu}\right\}$ satisfies the hypotheses of the theorem except that the constraint on the quantity of material only holds at the limit. More precisely, we have:

$$
F_{\varepsilon}^{\mu} \leq C_{\not}, \quad\left|Q_{\varepsilon}^{\mu}-S\right| \leq C_{\not \notin} \varepsilon
$$

Proof (of (5.2)) First, since by hypothesis $\mathscr{Q}\left(\sigma_{\varepsilon}, \nabla t_{\varepsilon}\right)=2 S \varepsilon$, we have

$$
Q_{\varepsilon}^{\mu}-S=\frac{1}{2 \varepsilon} \int_{\Omega_{\varepsilon}}\left(1 / \rho\left(\mu t_{\varepsilon}\right)-1\right) \sigma_{\varepsilon} \cdot \nabla t_{\varepsilon}
$$

From $\rho\left(\mu t_{\varepsilon}\right)=1+O(\varepsilon)$ and using the inequality $\left|1-\sigma_{\varepsilon} \cdot \nabla t_{\varepsilon}\right| \leq 2\left(f_{0}\left(\sigma_{\varepsilon}, \nabla t_{\varepsilon}\right)+\right.$ $\left.\sqrt{f_{0}\left(\sigma_{\varepsilon}, \nabla t_{\varepsilon}\right)}\right)($ see $(2.9))$ we deduce,

$$
\left|Q_{\varepsilon}^{\mu}-S\right| \leq C_{\mathscr{\varepsilon}}\left[\mathscr{F}_{0}\left(a_{\varepsilon}\right)+\mathscr{H}^{d}\left(\Omega_{\varepsilon}\right)\right] \leq C_{\dot{q}}\left(\varepsilon^{3}+\varepsilon Q_{\varepsilon}^{\prime}\right),
$$

where we have introduced the quantity $Q_{\varepsilon}^{\prime}:=\mathscr{H}^{d}\left(\Omega_{\varepsilon}\right) /(2 \varepsilon)$. Similarly,

$$
F_{\varepsilon}^{\mu} \leq C_{\not{q}}\left(\mathscr{F}_{0}\left(a_{\varepsilon}\right) / \varepsilon^{3}+\mathscr{H}^{d}\left(\Omega_{\varepsilon}\right) / \varepsilon\right) \leq C_{\not{\phi}}\left(1+Q_{\varepsilon}^{\prime}\right) .
$$


Next, from the estimate (2.3) of Lemma 2.1, we also have

$$
\left|Q_{\varepsilon}^{\prime}-Q_{\varepsilon}^{\mu}\right| \leq C_{\not \varepsilon} \varepsilon^{2}\left[F_{\varepsilon}^{\mu}+\sqrt{F_{\varepsilon}^{\mu} Q_{\varepsilon}^{\prime}}\right]
$$

Combining (5.3) and (5.5) we first deduce $Q_{\varepsilon}^{\prime} \leq S+C_{\dot{q}} \varepsilon^{3}\left(1+F_{\varepsilon}^{\mu}\right)$. With (5.4), this leads to $F_{\varepsilon}^{\mu} \leq C_{\not \notin}$ and $Q_{\varepsilon}^{\prime} \leq S+C_{\not \notin} \varepsilon^{3}$. Returning to (5.3) we obtain (5.2).

With minor adjustments in the proof, we see that $\left\{a_{\varepsilon}^{\mu}\right\}$ satisfies the conclusion of Theorem 1.1 and owing to $\sigma_{\varepsilon}=(1+O(\varepsilon)) \sigma_{\varepsilon}^{\mu}$, the same convergence properties hold for the initial family $\left\{a_{\varepsilon}\right\}$.

Eventually, let us show that under the above assumptions, the conclusion of Theorem 1.2 (lower bound) holds with $\mathscr{W}_{\mu}$ in place of $\mathscr{W}$. We have two choices. Either we go further with $a_{\varepsilon}^{\mu}$ as main unknown and consider the stored energy function $f_{\mu}(s, \sigma, \tau):=f(\rho(\mu s) \sigma, \tau)$ or we come back to the initial unknown $\sigma_{\varepsilon}$. In the first case, we substitute $\sigma_{\varepsilon}^{\mu}$ for $\sigma_{\varepsilon}$ in the definition of the approximate mean curvature, that is for $x \in \Sigma_{\varepsilon}$, we set

$$
\hat{h}_{\varepsilon}(x):=\frac{\chi_{(\varepsilon), \eta}(\varepsilon x)}{\varepsilon} \int_{\mathbf{R}^{d}} \sigma_{\varepsilon}^{\mu}(x+\varepsilon z) \cdot \pi_{v_{\varepsilon}(x)} \nabla_{y} \zeta\left(z ; v_{\varepsilon}(x)\right) d z .
$$

With this definition, Proposition 4.1 still holds. In particular, $\hat{h}_{\varepsilon}$ weakly approximates $h_{\varepsilon}$. Then, we define the energy of $a_{\varepsilon}^{\mu}$ as

$$
\mathscr{F}_{\mu}\left(t_{\varepsilon}, \sigma_{\varepsilon}, \nabla t_{\varepsilon}, \Omega_{\varepsilon}\right):=\int_{\Omega_{\varepsilon}} f_{\mu}\left(\sigma_{\varepsilon}^{\mu}, \nabla t_{\varepsilon}\right)=\int_{\Omega_{\varepsilon}} f\left(\sigma_{\varepsilon}, \nabla t_{\varepsilon}\right)=\mathscr{F}\left(\sigma_{\varepsilon}, \nabla t_{\varepsilon}, \Omega_{\varepsilon}\right) .
$$

We are led to study a new class of local problems associated with the energy density functions $f_{\mu}$ which depends on the additional variable $s$. This point of view is interesting as it opens the way to other alternative models. However, for the sake of brevity, we continue with the second method.

We still build the family of hypersurfaces $\left\{\Sigma_{\varepsilon}\right\}$ and related objects from the family $\left\{a_{\varepsilon}^{\mu}\right\}$. We also define the approximate mean curvature by (5.6). Then, we want to define an approximation of $h-\mu$. Let us try

$$
\begin{aligned}
\hat{h}_{\varepsilon}^{\prime}(x):= & \frac{\chi_{(\varepsilon), \eta}(\varepsilon x)}{\varepsilon} \int_{\mathbf{R}^{d}} \sigma_{\varepsilon}(x+\varepsilon z) \cdot \pi_{v_{\varepsilon}(x)} \nabla_{y} \zeta\left(z ; v_{\varepsilon}(x)\right) d z, \\
& =\frac{\chi_{(\varepsilon), \eta}(\varepsilon x)}{\varepsilon} \int_{\mathbf{R}^{d}}\left[\sigma_{\varepsilon}(x+\varepsilon z)-v_{\varepsilon}(x)\right] \cdot \pi_{v_{\varepsilon}(x)} \nabla_{y} \zeta\left(z ; v_{\varepsilon}(x)\right) d z
\end{aligned}
$$

which corresponds to the original definition of $\hat{h}$ in the case $\mu=0$. As already mentioned, the function $\hat{h}_{\varepsilon}$ is an approximation of $h_{\varepsilon}$. Now for $x \in \Sigma_{\varepsilon}$, we have

$$
\begin{aligned}
\hat{h}_{\varepsilon}^{\prime}(x) & -\hat{h}_{\varepsilon}(x) \\
& =\chi_{(\varepsilon), \eta}(\varepsilon x) \int_{\mathbf{R}^{d}} q_{\varepsilon}(x, z)\left[\sigma_{\varepsilon}(x+\varepsilon z)-v_{\varepsilon}(x)\right] \cdot\left(\zeta_{\perp} \nabla_{y} \zeta_{/ /}\right)\left(z ; v_{\varepsilon}(x)\right) d z
\end{aligned}
$$


with $q_{\varepsilon}(x, z):=\left[\rho\left(\mu t_{\varepsilon}(x+\varepsilon z)\right)-1\right] / \varepsilon$. Integrating by parts, we have,

$$
\begin{aligned}
& \hat{h}_{\varepsilon}^{\prime}(x)-\hat{h}_{\varepsilon}(x) \\
= & -\chi_{(\varepsilon), \eta}(\varepsilon x) \int_{\mathbf{R}^{d}} \nabla_{z}\left[q_{\varepsilon}(x, z) \zeta_{\perp}\left(z ; v_{\varepsilon}(x)\right)\right] \cdot\left[\sigma_{\varepsilon}^{\mu}(x+\varepsilon z)-v_{\varepsilon}(x)\right] \zeta_{/ /}\left(z ; v_{\varepsilon}(x)\right) d z .
\end{aligned}
$$

Now from $\left|\nabla_{z}\left[q_{\varepsilon}(x, z) \zeta_{\perp}\left(z ; v_{\varepsilon}(x)\right)\right]\right| \leq C\left(1+\left|\nabla t_{\varepsilon}\right|(x+\varepsilon z)\right)$, we deduce,

$$
\left|\hat{h}_{\varepsilon}^{\prime}(x)-\hat{h}_{\varepsilon}(x)\right| \leq C \chi_{(\varepsilon), \eta}(\varepsilon x)\left[\left[\mathscr{E}_{3}(x / \varepsilon)\right]^{1 / 2}+\left[\mathscr{E}_{3}(x / \varepsilon)\right]^{1 / 4}\right] .
$$

Integrating on $\Sigma_{\varepsilon}$, we deduce,

$$
\int_{\Sigma_{\varepsilon}}\left|\hat{h}_{\varepsilon}^{\prime}(x)-\hat{h}_{\varepsilon}(x)\right| \leq C_{\dot{q}}\left(\varepsilon+\varepsilon^{1 / 2}\right) \stackrel{\varepsilon \downarrow 0}{\longrightarrow} 0 .
$$

We conclude that $\hat{h}_{\varepsilon}^{\prime}$ is also a weak approximation of $h_{\varepsilon}$ and not of $h_{\varepsilon}-\mu$.

Let us now give a relevant definition of a weak approximation of $h_{\varepsilon}-\mu$. For $x \in \Sigma_{\varepsilon}$, we set

$$
\hat{h}_{\varepsilon}^{\mu}(x):=-\frac{\chi_{(\varepsilon), \eta}(\varepsilon x)}{\varepsilon} \int_{\mathbf{R}^{d}} \sigma_{\varepsilon}(x+\varepsilon z) \cdot \nabla_{y} \zeta_{\perp}\left(z ; v_{\varepsilon}(x)\right) \zeta_{/ /}\left(z ; v_{\varepsilon}(x)\right) d z .
$$

When $\mu=0$, we see (by integrating by parts) that this definition is the same as the definitions (5.6) or (5.7). In the general situation, an integration by parts leads to

$$
\hat{h}_{\varepsilon}^{\mu}(x)-\hat{h}_{\varepsilon}^{\prime}(x)=\chi_{(\varepsilon), \eta}(\varepsilon x) \int_{\mathbf{R}^{d}} \nabla_{z} \cdot\left[\frac{\sigma_{\varepsilon}(x+\varepsilon z)}{\varepsilon}\right] \zeta\left(z ; v_{\varepsilon}(x)\right) d z
$$

Form the constraint 5.1 we have

$$
\begin{aligned}
\nabla_{z} \cdot\left[\frac{\sigma_{\varepsilon}(x+\varepsilon z)}{\varepsilon}\right] & =\mu \rho^{\prime}\left(\mu t_{\varepsilon}\right)\left[\nabla t_{\varepsilon} \cdot \sigma_{\varepsilon}\right](x+\varepsilon z) \\
& =-\mu+\mu\left[1-\nabla t_{\varepsilon} \cdot \sigma_{\varepsilon}\right](x+\varepsilon z)+O(\varepsilon)\left|\nabla t_{\varepsilon} \cdot \sigma_{\varepsilon}\right|(x+\varepsilon z)
\end{aligned}
$$

Therefore,

$$
\left|\hat{h}_{\varepsilon}^{\mu}(x)-\hat{h}_{\varepsilon}^{\prime}(x)-\mu\right| \leq C(\xi)\left(\sqrt{\mathscr{E}_{3}(x / \varepsilon)}+\mathscr{E}_{3}(x / \varepsilon)+\varepsilon\right),
$$

and $\hat{h}_{\varepsilon}^{\mu}$ is a weak approximation of $h_{\varepsilon}-\mu$ in the sense of Proposition 4.1.

Now we can continue the proof of Theorem 1.2 by substituting $\hat{h}_{\varepsilon}^{\mu}$ for $\hat{h}_{\varepsilon}$ and by performing the respective substitution in the definition of the local problem, i.e. replacing $\hat{h}^{\sharp}$ by $\hat{h}^{\mu, \sharp}$ in Definition 4.3 with (4.18) replaced by

$$
\hat{h}^{\mu \sharp \sharp}\left(a^{\#}\right):=-\int_{\mathbf{R}^{d}} \sigma^{\#} \cdot\left(\zeta_{/ /} \nabla_{y} \zeta_{\perp}\right)\left(y ; e_{d}\right) d y .
$$

Then, in place of the identity (4.31), we have

$$
\int_{\mathbf{R}^{d}} \sigma_{\infty}^{b} \cdot\left(\zeta_{/ /} \nabla_{y} \zeta_{\perp}\right)\left(y ; e_{d}\right) d y=-1
$$


which yields, as before, the constraint (4.32) for the 2-dimensional final optimisation problem. No further changes are required.

Eventually, in order to pass the limit in $\mathscr{W}_{\mu}\left(\Sigma_{\varepsilon}\right)$ as $\varepsilon \downarrow 0$, we need to extend the definition of $\mathscr{W}_{\mu}$ as a lower semi-continuous fonctional defined on the set of oriented $(d-1)$ varifolds which admit a $L^{2}$ generalized mean curvature. With the notation of Appendix D, this is achieved by setting

$$
\mathscr{W}_{\mu}(\mathscr{V}):=\mathscr{W}(\mathscr{V})-2 \mu\langle\mathscr{V} ;(x, n) \mapsto H(x) \cdot n\rangle+\mu^{2}\langle\mathscr{V} ; 1\rangle .
$$

With this definition, we have (compare (D.7))

$$
\sqrt{\mathscr{W}_{\mu}(\mathscr{V})}=\|H-\mu n\|_{L^{2}\left(\mathbf{R}^{d, \mathscr{V}}\right)}=\sup \langle\mathscr{V} ; X \cdot(H-\mu n)\rangle
$$

where the supremum is taken over $\left\{X \in C_{c}^{1}\left(\mathbf{R}^{d} \times S^{d-1}\right) ;\|X\|_{L^{2}\left(\mathbf{R}^{d}, \mathscr{V}\right)} \leq 1\right\}$. We deduce that the functional $\mathscr{W}_{\mu}$ is lower semicontinuous with respect to the convergence of measures. For the elements of $\mathscr{A}_{0}$, we can write (compare (D.9)),

$$
\mathscr{W}_{\mu}\left(\mathscr{V}_{0}\right)=\int_{\Sigma_{0}}\left|H_{0}-\mu v_{0}\right|^{2}\left(\theta_{0}^{+}+\theta_{0}^{-}\right) d \mathscr{H}^{d-1} .
$$

As a conclusion, substituting (5.8) for the generalized Willmore functional, Theorem 1.2 extends to the case $\mu \neq 0$.

\section{A Proof of Lemma 2.3}

let us rephrase the lemma.

Lemma A.1 Let $t \in W_{l o c}^{1,2}\left(\mathbf{R}^{d}\right)$, let $O$ be a bounded open subset of $\mathbf{R}^{d}$ and let $u$ be the variational harmonic extension of $t$ in $O$. We assume that there exists $\delta>0$ and a (concave) modulus of continuity $\omega$ such that $O$ has the exterior ball property with radius $\delta$ and that $t$ is $\omega$-continuous with values into $[-1,1]$. Then there exists a modulus of continuity $\omega^{\star}$ only depending on $d, \delta$ and $\omega$ such that $u$ is $\omega^{\star}$-continuous in $\mathbf{R}^{d}$.

Proof We start by regularizing the boundary data $t$ and the domain. For $0<\eta \leq$ $\delta / 2$, we set $t_{\eta}^{\prime}:=t \star \rho_{\eta}$ where $\rho_{\eta}=\eta^{-d} \rho(\cdot / \eta)$ is a standard non-negative mollifier, compactly supported in $B_{\eta}$. We also set $O_{\eta}^{\prime}=O+B_{\eta} \supset O$.

Writing $t_{\eta}^{\prime}(y)-t_{\eta}^{\prime}(z)=\int[t(y-x)-t(z-x)] d \rho_{\eta}(x) \leq \int \omega(y-z) d \rho_{\eta}=\omega(y-z)$, we see that the functions $\left\{t_{\eta}^{\prime}\right\}_{0<\eta<\delta / 2}$ are $\omega$-continuous. They are also uniformly bounded in $W_{l o c}^{1,2}\left(\mathbf{R}^{d}\right)$ and satisfy $-1 \leq t_{\eta}^{\prime} \leq 1$.

By construction, the domain $O_{\eta}^{\prime}$ satisfies the exterior ball condition for balls with radii $\delta / 2$ and the interior ball condition for balls with radii $\eta$. In particular $\partial O_{\eta}^{\prime}$ has $C^{1,1}$ regularity and $O_{\eta}^{\prime}$ is a bounded Lipschitz domain.

Let $u_{\eta}^{\prime}$ be the variational harmonic extension of $t_{\eta}^{\prime}$ in $O_{\eta}^{\prime}$, defined as

$$
u_{\eta}^{\prime}:=\operatorname{argmin}\left\{\int_{B_{R / \varepsilon}}|\nabla \varphi|^{2}: \varphi \in W_{l o c}^{1,2}\left(\mathbf{R}^{d}\right), \varphi \equiv t_{\eta}^{\prime} \text { a.e on } \mathbf{R}^{d} \backslash O_{\eta}^{\prime}\right\} .
$$




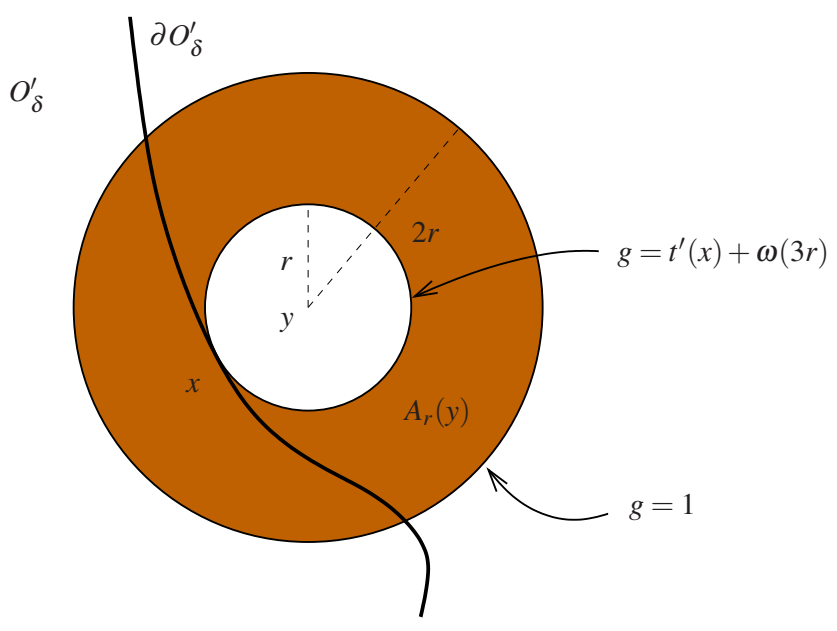

Fig. A.1 The barrier function $g$.

By classical elliptic regularity theory, $u_{\eta}^{\prime}$ is of class $C^{1}$ in $\overline{O_{\eta}^{\prime}}$ and $u_{\eta}^{\prime} \equiv t_{\eta}^{\prime}$ on $\partial O_{\eta}^{\prime}$. We claim that

$\exists$ a modulus of continuity $\omega^{\prime}=\omega^{\prime}(\omega, \delta, d)$ such that $u_{\eta}^{\prime} \in C_{\omega^{\prime}}\left(\overline{O_{\delta, \eta}^{\prime}}\right)$.

Assuming the claim, there exists a function $u^{\prime} \in W^{1,2}\left(B_{R / \varepsilon}\right)$ such that, up to extraction, $u_{\eta}^{\prime} \rightarrow u^{\prime}$ as $\eta \downarrow 0$. Moreover, $u^{\prime}$ is harmonic in $O$, is $\omega^{\prime}$-continuous in $\bar{O}$ and $u^{\prime} \equiv t$ in $\mathbf{R}^{d} \backslash \bar{O}$. Hence $u=u^{\prime}$ and $u$ is $\omega^{\prime}$-continuous in $\bar{O}$. Since $u=t$ is $\omega$-continuous in $\mathbf{R}^{d} \backslash \bar{O}$, we conclude that $u$ is $\left(\omega+\omega^{\prime}\right)$-continuous in $\mathbf{R}^{d}$ and the lemma is proved.

Let us now establish (A.1). We fix $\eta \in(0, \delta / 2]$ and drop the subscripts $\eta$.

Step 1 (interior estimate) First, by the maximum principle, we have in any case $\left|u^{\prime}(y)-u^{\prime}(z)\right| \leq 2$. Now, let $r \in(0,1 / 2]$ and $B_{r}(x) \subset O^{\prime}$. By harmonic regularity we have $|\nabla u| \leq C / r$ in $B_{r / 2}(x)$. So $\left|u^{\prime}(y)-u^{\prime}(z)\right| \leq C \sqrt{|y-z|}$ for every $y, z \in B_{r^{2}}(x)$.

From this estimate, we conclude that we only have to establish that for any $x \in \partial O^{\prime}, u^{\prime}(z) \rightarrow u^{\prime}(x)$ as $z \in O_{\delta}^{\prime} \rightarrow x$ with a convergence rate that only depends on $\omega, \delta$ and $d$.

Step 2 (continuity up to the boundary) Let us fix $x \in \partial O_{\delta}^{\prime}$ and let $r \in(0, \delta / 2]$. By hypothesis, there exists a ball $B_{r}(y) \subset \mathbf{R}^{d} \backslash \overline{O^{\prime}}$ such that $x \in \partial B_{r}(y)$.

As a barrier, we consider the harmonic function $g$ defined on the ring $A_{r}(y):=$ $B_{2 r}(y) \backslash \overline{B_{r}}(y)$ and satisfying the boundary conditions $g \equiv t^{\prime}(x)+\omega(3 r)$ on $\partial B_{r}(y)$ and $g \equiv 1$ on $\partial B_{2 r}(y)$ (see Figure A.1).

We claim that $g \geq u^{\prime}$ in $A_{r}(y) \cap O_{\delta}^{\prime}$. Indeed, $g-u^{\prime}$ is harmonic on this set, so by the maximum principle it is sufficient to check the inequality on $\partial\left(A_{r}(y) \cap O^{\prime}\right)$. This boundary splits into:

$$
\partial\left(A_{r}(y) \cap O^{\prime}\right)=\left(\partial B_{2 r}(y) \cap \overline{O^{\prime}}\right) \cup\left(\overline{A_{r}(y)} \cap \partial O^{\prime}\right) .
$$


On the first part, we have $g-u^{\prime}=1-u^{\prime} \geq 0$. On the second part $u^{\prime}=t^{\prime}$ on $\partial O^{\prime}$, so, $g(z)-u^{\prime}(z)=t^{\prime}(x)+\omega(3 r)-t^{\prime}(z) \geq \omega(3 r)-\omega(|x-z|) \geq 0$ since $|x-z| \leq 3 r$ and $t^{\prime}$ is $\omega$-continuous. Hence, $g \geq u^{\prime}$ in $A_{r}(y) \cap O^{\prime}$.

An explicit computation gives

$$
g(z)=t^{\prime}(x)+\omega(3 r)+\left(1-t^{\prime}(x)-\omega(3 r)\right) \frac{G(1)-G(|z-y| / r)}{G(1)-G(2)},
$$

where $z \mapsto G(|z|)$ denotes a radially symmetric decreasing multiple of the fundamental solution of the Laplacian $\left(G(s)=-\ln s\right.$ if $d=2$ and $G(s)=s^{2-d}$ for $d \geq 3)$. Hence, for every $x \in \partial O^{\prime}, r \in(0, \delta / 2)$ and $z \in O^{\prime} \cap B_{r}(x)$, we have

$$
u^{\prime}(z)-t^{\prime}(x) \leq g(z)-t^{\prime}(x) \leq \omega(3 r)+2 \frac{G(1)-G(|z-y| / r)}{G(1)-G(2)}
$$

Substituting $-t^{\prime}$ for $t$ and $-u^{\prime}$ for $u$, we obtain the corresponding result with opposite signs. We conclude that for every $x \in \partial O^{\prime}, r \in(0, \delta / 2)$ and $z \in O^{\prime} \cap B_{r}(x)$,

$$
\left|u^{\prime}(z)-t^{\prime}(x)\right| \leq \omega(3 r)+2 \frac{G(1)-G(|z-y| / r)}{G(1)-G(2)} .
$$

In particular, for $x \in \partial O^{\prime}$ and $z \in O^{\prime}$, such that $|z-x| \leq \delta^{2}$, we can choose $r=$ $\sqrt{|x-z|}$. In this case,

$$
\frac{|z-y|}{r} \leq \frac{|x-y|}{r}+\frac{|z-x|}{r}=1+\sqrt{|z-x|} .
$$

This leads to,

$$
\left|u^{\prime}(z)-t^{\prime}(x)\right| \leq \omega(3 \sqrt{|x-z|})+2 \frac{G(1)-G(1+\sqrt{|z-x|})}{G(1)-G(2)} \stackrel{|z-x| \downarrow 0}{\longrightarrow} 0 .
$$

This establishes the claim (A.1).

\section{B Proofs of Lemmas 2.4, 2.5 and 2.6}

\section{B.1 proof of Lemma 2.4}

(a) Without loss of generality, we assume $z=0$ and $\xi \in(0,1 / 20)$. Let $\eta>0$. We establish by contradiction that (a) holds for $\beta>0$ small enough.

If (a) were not true for any value of $\beta>0$, there would exist two sequences $\left(a_{k}\right),\left(a_{k}^{\prime}\right)$ with $a_{k}=\left(\sigma_{k}, \nabla t_{k}, O_{k}\right), a_{k}^{\prime}=\left(\sigma_{k}, \nabla u_{k}, O_{k}^{\prime}\right)$ such that:

(i) $\left(a_{k}\right)$ and $\left(a_{k}^{\prime}\right)$ both satisfy the hypotheses of Theorem I.1.1 with $O=B_{3}$. In particular, $\left(u_{k}\right)$ and $\left(t_{k}\right)$ are uniformly equicontinuous.

(ii) Either $\left|\nabla u_{k}\right|(0)<1 / 2$;

or $\left|\nabla u_{k}\right|(0) \geq 1 / 2$ and using the notation

$$
\bar{n}_{k}:=\nabla u_{k} /\left|\nabla u_{k}\right|(0), \quad D_{k}:=-u_{k}(0) \bar{n}_{k}+D_{1}^{\xi}\left(\bar{n}_{k}\right), \quad \varphi_{k}(y)=u_{k}(0)-z \cdot \bar{n}_{k},
$$

we have either: 
- $D_{k} \not \subset O_{k} \cap O_{k}^{\prime}$

- or $D_{k} \subset O_{k} \cap O_{k}^{\prime}$ and

$$
\left\|t_{k}-\varphi_{k}\right\|_{L^{\infty}\left(\bar{D}_{k}\right)}+\left\|u_{k}-\varphi_{k}\right\|_{L^{\infty}\left(\bar{D}_{k}\right)}+\left\|\nabla t_{k}-\nabla \varphi_{k}\right\|_{L^{2}\left(D_{k}\right)}>\eta ;
$$

(iii) Moreover, $\left|u_{k}\right|(0) \leq 1 / 2$ and setting $\widetilde{F}_{k}:=\left[\left|t_{k}\right| \geq 9 / 10\right]+\overline{B_{\delta}}$, we have (taking into account (2.10)) $u_{k} \equiv t_{k}$ on $\widetilde{F}_{k}$ and $u_{k}$ is harmonic in $B_{3} \backslash \widetilde{F}_{k}$.

First, up to extraction, we may assume that both $\left(t_{k}(0)\right)_{k}$ and $\left(u_{k}(0)\right)_{k}$ converge towards respectively $s$ and $s^{\prime}$ with $|s| \leq 9 / 10$ and $\left|s^{\prime}\right| \leq 1 / 2$. By Theorem I.1.1 there exists $\bar{n}, \bar{n}^{\prime} \in S^{d-1}$ such that

$$
t_{k} \stackrel{k \uparrow \infty}{\longrightarrow} \varphi \text { on } C_{l o c}\left(O_{\star}\right), \quad u_{k} \stackrel{k \uparrow \infty}{\longrightarrow} \varphi^{\prime} \text { on } C_{l o c}\left(O_{\star}^{\prime}\right),
$$

with $\varphi(y):=s+\bar{n} \cdot y, \varphi^{\prime}(y):=s^{\prime}+\bar{n}^{\prime} \cdot y, O_{\star}=B_{2} \cap[|\varphi|<1], O_{\star}^{\prime}=B_{2} \cap\left[\left|\varphi^{\prime}\right|<1\right]$.

Now, for any $k \geq 1, u_{k}$ is harmonic in $B_{\delta_{\xi}^{\star}}$. By harmonic regularity, we see that $\nabla u_{k}(0) \rightarrow \nabla \varphi^{\prime}(0)=\bar{n}^{\prime}$. In particular, for $k$ large enough, there holds

$$
\left|\nabla u_{k}\right|(0) \geq 1 / 2 \text {. }
$$

Next, considering the segment $I=\{y+(r-s) \bar{n}:-1<r<1\} \subset O_{\star}^{\prime}$, and the mapping $p:(-1,1) \rightarrow I$ defined by $p(r)=y+(r-s) \bar{n}$, we have $u_{k} \circ p \rightarrow I d_{(-1,1)}$ as $k \uparrow \infty$.

In particular, for $k$ large enough $\left|u_{k}\right|>9 / 10$ on $p(19 / 20,1)$ which implies $t_{k} \equiv u_{k}$ on $p(19 / 20,1)$.

Passing to the limit we conclude that $\bar{n}=\bar{n}^{\prime}, s=s^{\prime}$ and $\varphi=\varphi^{\prime}$. By uniform equicontinuity of the sequence $\left(t_{k}\right)$, and convergence of $\left(t_{k}\right)$ towards $\varphi^{\prime}$ on $O_{\star}^{\prime}$ we then see that

$$
\widetilde{D}_{k}:=-u_{k}(0) \bar{n}_{k}+D_{1+\xi}^{\xi / 2}\left(\bar{n}_{k}\right) \subset\left[\left|t_{k}\right|<1-\xi / 2\right], \quad \text { for } k \text { large enough. }
$$

We already know that the $L^{\infty}$ norms in (B.1) converge to 0 .

Applying the weak rigidity inequality of Theorem I.2.2 to $t_{k}$ in $\widetilde{D}_{k} \supset D_{k}$ we see that the $W^{1,2}$-seminorms also converge to 0 which contradicts (B.1). This proves (a).

(b) Part (b) follows from (a), the harmonicity of $u$ in the domain $[u<4 / 5]$ and the implicit function theorem. However, we use below a fixed point method (somehow reproving the implicit function theorem).

We assume $\mathscr{E}_{3}(z) \leq \beta_{a}\left(\xi, \eta^{\prime}\right)$ where $\beta_{a}(\xi, \eta)$ denotes the positive constant provided by part (a) and where $\eta^{\prime} \in(0, \eta)$ will be fixed later. As above we assume $\xi<1 / 20$, we also assume without loss of generality $z_{0}=0$ and $n(z)=e_{d}$ so that $\varphi(y)=y_{d}$ and $D=D_{1}^{\xi}$.

We have to show that there exists an analytic function $\Psi: D_{1}^{\prime} \times(-1 / 2,1 / 2) \rightarrow \mathbf{R}$ with $\|\Psi\|_{C^{2}} \leq \eta$ such that for every $(y, s) \in D_{1}^{\xi} \times(-1 / 2,1 / 2)$, there holds

$$
u(y)-s=0 \quad \Longleftrightarrow \quad y_{d}-s=\Psi\left(y^{\prime}, s\right) .
$$


Step 1. Let us first notice that we can restrict the problem to a domain in which $u$ is harmonic. Indeed, from part (a) we have for every $y \in D_{1}^{\xi} \backslash D_{1}^{3 / 8}$ and $|s|<1 / 2$

$$
|u(y)-s| \geq|\varphi(y)-s|-|u(y)-\varphi(y)|>1 / 8-\|u-\varphi\|_{L^{\infty}\left(D_{1}^{\xi}\right)} \geq 1 / 8-\eta^{\prime} .
$$

Assuming $\eta^{\prime} \leq 1 / 8$, the left hand side of (B.2) can only hold if $y \in D_{1}^{3 / 8}$. Now in this set, we have

$$
|u(y)| \leq 5 / 8+\|u-\varphi\|_{L^{\infty}\left(D_{1}^{\xi}\right)} \leq 5 / 8+\eta^{\prime} \leq 3 / 4 .
$$

Since $u$ is harmonic in $[|u|<4 / 5]$, we see that, by $\omega_{\dot{\alpha}}^{\star}$-continuity, $u$ is harmonic $D_{1}^{3 / 8}+B_{\rho}$ for some $\rho>0$ only depending on $\omega_{\dot{q}}^{\star}$. Also notice that for the same reason we may assume

$$
\|u-\varphi\|_{L^{\infty}\left(D_{1}^{3 / 8}+B_{\rho}\right)} \leq 2 \eta^{\prime}
$$

In summary, we only have to establish (B.2) for $(y, s) \in D_{1}^{3 / 8} \times(-1 / 2,1 / 2)$ and we can assume that $u$ is harmonic in $D_{1}^{3 / 8}+B_{\rho}$ and satisfies (B.3).

Step 2. Noting, $z_{d}=y_{d}-s$ and $\theta\left(y^{\prime}, s, z_{d}\right):=[u-\varphi]\left(y^{\prime}, z_{d}+s\right)$, (B.2) rewrites as the fixed point problem

$$
z_{d}=-\theta\left(y^{\prime}, s, z_{d}\right) \quad \Longleftrightarrow \quad z_{d}=\Psi\left(y^{\prime}, s\right),
$$

in the convex domain $Q:=\left\{\left(y^{\prime}, s, z_{d}\right): y^{\prime} \in D_{1}^{\prime},|s|<1 / 2,\left|z_{d}+s\right|<5 / 8\right\}$. Now by (B.3) $\theta$ maps $Q$ into $\left[-2 \eta^{\prime}, 2 \eta^{\prime}\right]$ and by harmonic regularity we have

$$
\left\|D^{k} \theta\right\|_{L^{\infty}(Q)} \leq C(k) \eta^{\prime} / \rho^{k}
$$

Assuming $C(1) \eta^{\prime} / \rho \leq 1 / 2$, we have in particular

$$
\left\|\partial_{z_{d}} \theta\right\|_{L^{\infty}(Q)} \leq 1 / 2
$$

Therefore applying Picard's iteration to (B.4), we obtain for every $\left(y^{\prime}, s\right) \in D_{1}^{1 / 2}$ the existence of a unique $z_{d}=: \Psi\left(y^{\prime}, s\right)$ satisfying (B.4). Moreover, we deduce from (B.5) that $\Psi$ is smooth with

$$
\left\|D^{k} \Psi\right\|_{L^{\infty}\left(D_{1}^{1 / 2}\right)} \leq C(k) \eta^{\prime} / \rho^{k} \quad \text { for } k \geq 0 .
$$

Hence assuming $\eta^{\prime}>0$ small enough we have, $\|\Psi\|_{C^{2}} \leq \eta$. Eventually, using again (B.6), we can apply the analytic implicit function theorem to $\Phi\left(y^{\prime}, s, z_{d}\right):=$ $z_{d}+\theta\left(y^{\prime}, s, z_{d}\right)$ in the neighborhood of each point $\left(y^{\prime}, s, \Psi\left(y^{\prime}, s\right)\right)$. We deduce that $\Psi$ is analytic which ends the proof of (b).

(c) This point easily follows from (b). 
B.2 proof of Lemma 2.5

Part (a) is a direct consequence of the preceding lemma. Part (b) follows from Corollary I.3.1 and the harmonicity of $u$ in $B_{\delta_{\xi}^{\star}}$.

Let us establish part (c). Without loss of generality, we assume $x=0$ and $\xi \in(0,1 / 20)$. We apply Theorem I.2.2 to the function $u$ in the cylinder $D_{\text {int }} \subset D$ and in $B_{\delta_{\mho^{\star}}} \subset D$. There exists $e_{\star}^{\prime}, e_{\star} \in S^{d-1}$ such that

$$
\begin{aligned}
& \int_{D_{\text {int }}}\left|\nabla u-e_{\star}^{\prime}\right|^{2} \leq C(\xi) \sqrt{\mathscr{E}(D)}, \\
& \int_{B_{\delta_{\xi}^{\star}}}\left|\nabla u-e_{\star}\right|^{2} \leq C\left(\delta_{\not{q}}^{\star}\right) \sqrt{\mathscr{E}(D)} .
\end{aligned}
$$

In particular, we have $\left|e_{\star}-e_{\star}^{\prime}\right|^{2} \leq C\left(\xi, \delta_{\dot{\xi}}^{\star}\right) \sqrt{\mathscr{E}(D)}$. Moreover since $u$ is harmonic in $B_{\delta_{\xi}^{\star}}$, we see that by the mean value property we can choose $e_{\star}=n(0)=: \bar{n}-$ as in the proof of Corollary I.3.1. Inequality (B.7) then implies

$$
\int_{D_{\text {int }}}|\nabla u-\bar{n}|^{2} \leq C\left(\xi, \delta_{\not{q}}^{\star}\right) \sqrt{\mathscr{E}(D)} .
$$

Now, let us apply Theorem I.3.1 to $u$ in $D_{\text {int }} \subset D$. There exists a harmonic function $\psi_{1} \in L^{2}\left(D_{\text {int }}\right)$ such that $\bar{n} \cdot \nabla \psi_{1} \equiv 0$ and

$$
\int_{D_{\text {int }}}\left|\nabla u-\bar{n}-\nabla \psi_{1}\right|^{2} \leq C\left(\xi, \delta_{\dot{q}}^{\star}\right) \mathscr{E}(D)
$$

Now, notice that by orthogonality, we have

$$
\left|\nabla \psi_{1}\right|^{2}(0)+|\nabla u(0)-\bar{n}|^{2}=\left|\nabla u(0)-\nabla \psi_{1}(0)-\bar{n}\right|^{2} \stackrel{(\mathrm{B} .9)}{\leq} C\left(\xi, \delta_{\dot{\phi}}^{\star}\right) \mathscr{E}(D) .
$$

In particular,

$$
\left|\nabla \psi_{1}\right|^{2}(0) \leq C\left(\xi, \delta_{\not{\phi}}^{\star}\right) \mathscr{E}(D) .
$$

Eventually, setting $\psi(y):=\psi_{1}(y)-y \cdot \nabla \psi_{1}(0)$, the function $\psi$ is harmonic in $D_{\text {int }}$, it satisfies $\bar{n} \cdot \nabla \psi \equiv 0$ and (2.13) holds thanks to (B.9) and (B.10). Inequality (2.12) then follows from (2.13) and (B.8).

\section{B.3 proof of Lemma 2.6}

We can prove the lemma by arguing by contradiction as in the proof of Lemma 2.4. The only difference is that we have to take into account all the connected components of $\left\{y: \lim _{k \rightarrow \infty} u_{k}(y) \in(-1,1)\right\}$ (see Figure I.1.4). Details are left to the reader. 


\section{Proof of Lemma 3.1}

We assume that $f$ satisfies (1.1) and (1.6), (1.7). Writing $D^{2} f(e, e)$ in the basis,

$$
\mathscr{B}=\left(\left(b_{1}, 0\right), \cdots,\left(b_{d-1}, 0\right),\left(0, b_{1}\right), \cdots,\left(0, b_{d-1}\right),(e, 0),(0, e)\right),
$$

we have

$$
D^{2} f(e, e)=\left(\begin{array}{ccc}
A & B & M_{1} \\
B^{T} & C & M_{2} \\
M_{1}^{T} & M_{2}^{T} & L
\end{array}\right)
$$

where $A, B, C, M_{1}, M_{2}, L$ are real matrices, $A, B, C \in \mathscr{M}_{d-1, d-1}(\mathbf{R}), M_{1}, M_{2} \in$ $\mathscr{M}_{d-1,2}(\mathbf{R}), L \in \mathscr{M}_{2,2}(\mathbf{R})$ and $A, C$ and $L$ are symmetric.

Let us consider the action of rotations with axis $e$ : for every $Q \in \mathrm{SO}(d-1)$ and every $x, y \in e^{\perp}=\mathbf{R}^{d-1} \subset \mathbf{R}^{d}$, we have $f((e+Q x),(e+Q y))=f((e+x),(e+y))$, which yields,

$$
D^{2} f(e, e)=\left(\begin{array}{ccc}
Q^{T} A Q & Q^{T} B Q & M_{1} Q \\
Q^{T} B^{T} Q & Q^{T} C Q & M_{2} Q \\
\left(M_{1} Q\right)^{T} & \left(M_{2} Q\right)^{T} & L
\end{array}\right) \quad \text { for every } Q \in \operatorname{SO}(d-1) .
$$

Identifying this expression with (C.1), we see that $M_{1}=M_{2}=0$ and that for $K=$ $A, B, C$ we have

$$
Q^{T} K Q=K \quad \text { for every } Q \in \mathrm{SO}(d-1),
$$

Let us split $B$ into its symmetric and skew-symmetric parts: $B=B_{s y}+B_{s k}$, so that (C.2) also holds for $K=B_{s y}$ and $K=B_{s k}$. Since $A, B_{s y}$ and $C$ are symmetric, hence diagonal in some orthonormal basis, this implies that these operators are diagonal in any orthonormal basis. Hence, there exists $a, b, c \in \mathbf{R}$ such that

$$
A=a \mathbf{I}_{d-1}, \quad B_{s y}=b \mathbf{I}_{d-1}, \quad C=c \mathbf{I}_{d-1} .
$$

At this point, in the basis $\mathscr{B}, D^{2} f(e, e)$ writes

$$
D^{2} f(e, e)=\left(\begin{array}{ccc}
a \mathbf{I}_{d-1} & b \mathbf{I}_{d-1}+B_{s k} & 0 \\
b \mathbf{I}_{d-1}-B_{s k} & c \mathbf{I}_{d-1} & 0 \\
0 & 0 & L
\end{array}\right) .
$$

Now, for a fixed $z \in S^{d-1} \cap e^{\perp}$, we observe that $d\left((e, e)+r(z, z), \mathbb{S}^{d-1}\right)=$ $O\left(r^{2}\right)$. Since $f$ is minimal on $\mathbb{S}^{d-1}$, we have $f((e, e)+r(z, z))=O\left(r^{4}\right)$ and the second order derivative of $r \mapsto f((e, e)+r(z, z))$ vanishes at $r=0$. From the last expression for $D^{2} f(e, e)$, this second order derivative is equal to $a|z|^{2}+b|z|^{2}+$ $z^{T} B_{s k} z+b|z|^{2}-z^{T} B_{s k} z+c|z|^{2}=a+2 b+c$. Hence

$$
b=-(a+c) / 2 \text {. }
$$

Next, the space $T=\operatorname{span}\{(e, 0),(0, e)\}^{\perp}$ is stable under the action of the symmetric operator $D^{2} f(e, e)$. Since this operator is non-negative, for every $v \in T$ 
we have $v^{T} D^{2} f(e, e) v \geq 0$. Choosing $v=\left(z, \lambda z^{\prime}\right)$ with $z, z^{\prime} \in e^{\perp}$, this inequality reads

$$
c\left|z^{\prime}\right|^{2} \lambda^{2}+2\left(b z \cdot z^{\prime}+z^{T} B_{s k} z^{\prime}\right) \lambda+a|z|^{2} \geq 0 \quad \text { for every } \lambda \in \mathbf{R} .
$$

Choosing $z=z^{\prime} \in S^{d-1} \cap e^{\perp}$, the term $z^{T} B_{s k} z^{\prime}$ vanishes and we end up with the condition $c \lambda^{2}+2 b \lambda+a \geq 0$ for every $\lambda \in \mathbf{R}$. This leads to $b^{2} \leq a c$ which together with the identity $b=-(a+c) / 2$ yields

$$
a=c=-b .
$$

Let us now establish that $B_{s k}=0$. Since $B_{s k} \in \mathscr{M}_{d-1, d-1}(\mathbf{R})$ is skew-symmetric, we have $B_{s k}=0$ if $d=2$. Now, we assume $d \geq 3$ and we apply (C.3) with $\lambda=1$, $z \in S^{d-1} \cap e^{\perp}$ and $z^{\prime}=z+r y$ for some $y \in e^{\perp} \cap z^{\perp}$. We deduce $z^{T} B_{s k} y=O(r)$. Hence, $z^{T} B_{s k} y=0$ for every $y \in e^{\perp} \cap z^{\perp}$ which yields the conclusion:

$$
B_{s k}=0 .
$$

We have established that $D^{2} f(e, e)$ has the form stated in the lemma. The nonnegativity of $a$ and $L$ follow from that of $D^{2} f(e, e)$. Eventually, assuming $f \geq \kappa f_{0}$ for some $\kappa>0$, we obtain $D^{2} f(e, e) \geq \kappa D^{2} f_{0}(e, e)$ which easily yields $a>0$ and $L$ positive definite.

\section{Oriented varifolds with $L^{2}$-generalized mean curvature}

We gather here well known facts about oriented integer varifolds. In particular, we extend the Willmore functional to the elements of $\mathscr{A}_{0}$.

Let us first introduce a suitable generalization of Definition 1.1.b extending the notion of hypersurface.

Definition D.1 (oriented integer rectifiable $(d-1)$-varifolds)

a) Given:

i. a $(d-1)$ rectifiable set $\Sigma \subset \mathbf{R}^{d}$, that is:

- $\mathscr{H}^{d-1}(\Sigma)<\infty$ and $\Sigma$ is $\mathscr{H}^{d-1}$-measurable,

- there exists a countable set of Lipschitz continuous functions $L_{k}: \mathbf{R}^{d-1} \rightarrow$ $\mathbf{R}^{d}$, such that $\mathscr{H}^{d-1}\left(\Sigma \backslash \cup_{k} L_{k}\left(\mathbf{R}^{d-1}\right)\right)=0$;

ii. a measurable unit normal $v$, that is $v: \Sigma \rightarrow S^{d-1}$ is $\mathscr{H}^{d-1}$-measurable and for almost every $x_{0} \in \Sigma$ and every $\varphi \in C_{c}\left(\mathbf{R}^{d}\right)$, there holds:

$$
\lambda^{d-1} \int_{\Sigma} \varphi\left(\lambda\left(\cdot-x_{0}\right)\right) d \mathscr{H}^{d-1} \stackrel{\lambda \uparrow \infty}{\longrightarrow} \theta\left(x_{0}\right) \int_{v^{\perp}\left(x_{0}\right)} \varphi d \mathscr{H}^{d-1}
$$

iii. two positive integer valued $\mathscr{H}^{d-1}$-integrable functions $\theta^{-}, \theta^{+}: \Sigma \rightarrow \mathbf{N} \backslash\{0\}$. 
We define the oriented $(d-1)$-varifold $\mathscr{V}=\mathscr{V}\left(\Sigma, v, \theta^{+}, \theta^{-}\right)$as

$$
\langle\mathscr{V} ; \varphi\rangle:=\int_{\Sigma}\left\{\theta^{+}(x) \varphi(x, v(x))+\theta^{-}(x) \varphi(x,-v(x))\right\} d \mathscr{H}^{d-1}(x) .
$$

b) The set of all such oriented integer rectifiable $(d-1)$-varifolds is denoted by $\mathbf{I V}^{o}$. In the sequel, we only write integer varifold for oriented integer rectifiable $(d-1)$-varifold. We also introduce the set

$$
\mathbf{I V}_{0}^{o}:=\left\{\mathscr{V} \in \mathbf{I V}^{o}: \Lambda \mathscr{V}=\nabla U \text { for some } U \in \mathscr{D}^{\prime}\left(\mathbf{R}^{d}\right)\right\}
$$

Equivalently, this set is formed by integer varifolds whose associated current has vanishing boundary (see Remark 1.2.b).

\section{Remark D.1}

$i$. The total mass of $\mathscr{V}=\mathscr{V}\left(\Sigma, v, \theta^{+}, \theta^{-}\right)$is $\langle v ; \mathbf{1}\rangle=\int_{\Sigma}\left(\theta^{+}+\theta^{-}\right) d \mathscr{H}^{d-1}$ and $\Lambda \mathscr{V}=\left(\theta^{+}-\theta^{-}\right) v \mathscr{H}^{d-1}\llcorner\Sigma$.

ii. The functions $\theta^{+}, \theta^{-}$account for multiplicity: the above formula means that the generalized surface pass at point $x \in \Sigma, \theta^{+}$times with orientation $v(x)$ and $\theta^{-}(x)$ times with opposite orientation.

iii. By continuity of the mapping $\Lambda: \mathscr{M}\left(\mathbf{R}^{d} \times S^{d-1}\right) \rightarrow \mathscr{D}^{\prime}\left(\mathbf{R}^{d}\right)^{d}$, the space $\{\mathscr{V} \in$ $\left.\mathscr{M}\left(\mathbf{R}^{d} \times S^{d-1}\right): \Lambda \mathscr{V} \equiv 0\right\}$ is closed in $\mathscr{M}\left(\mathbf{R}^{d} \times S^{d-1}\right)$.

Another important tool in the context of area minimization is the first variation $\delta \mathscr{V}(X)$ of a $(d-1)$-varifold. If $\mathscr{V}=\mathscr{V}(\Sigma, v)$ is the oriented $(d-1)$ varifold associated with a smooth hypersurface $\Sigma=\partial O$, this quantity describes the initial rate of change of the total mass of $\mathscr{V}$ under the flow generated by $X \in \mathscr{D}\left(\mathbf{R}^{d}, \mathbf{R}^{d}\right)$. It is defined as

$$
\delta \mathscr{V}(X):=\int_{\Sigma} \operatorname{div}_{\Sigma} X d \mathscr{H}^{d-1}
$$

Recall that for every $x \in \Sigma$, if $\left(e_{1}, \cdots, e_{d-1}\right)$ denotes an orthonormal basis of $v(x)^{\perp}$, we have

$$
\operatorname{div}_{\Sigma} X(x)=\sum_{i=1}^{d-1} e_{i}^{T} \nabla X(x) e_{i} .
$$

The right hand side of (D.1) naturally extends as a linear continuous functional defined on the space of $(d-1)$-varifolds: for every $\mathscr{V} \in \mathscr{M}\left(\mathbf{R}^{d} \times S^{d-1}\right)$, we set

$$
\delta \mathscr{V}(X):=\left\langle\mathscr{V} ;(y, n) \mapsto \operatorname{div}_{n^{\perp}} X(y)\right\rangle, \quad \text { for every } X \in \mathscr{D}\left(\mathbf{R}^{d}, \mathbf{R}^{d}\right) .
$$

In general, $\delta \mathscr{V}$ is a distribution of $\mathscr{D}^{\prime}\left(\mathbf{R}^{d}\right)^{d}$. However, if $\Sigma \subset \mathbf{R}^{d}$ is a smooth $(d-1)$-submanifold with tangent hyperplane $v^{\perp}(x)$, then the first variation of $\mathscr{V}=\mathscr{V}(\Sigma, v)$ can be related to the vectorial mean curvature $H: \Sigma \rightarrow \mathbf{R}^{d}$ defined as $H=h v=(-\nabla \cdot v) v$. The vector field $H$ only depends on the local geometry of $\Sigma$ : it does not depend on the local orientation $\pm v$. With this notation, the tangential Green formula reads,

$$
-\int_{\Sigma} H \cdot X d \mathscr{H}^{d-1}=\int_{\Sigma} \operatorname{div}_{\Sigma} X d \mathscr{H}^{d-1} \text { for every } X \in \mathscr{D}\left(\mathbf{R}^{d}, \mathbf{R}^{d}\right) .
$$


In particular, the first variation of $\mathscr{V}$ is a measure given by

$$
\delta \mathscr{V}(X)=-\int_{\Sigma} H \cdot X d \mathscr{H}^{d-1}
$$

Now let $R, S>0$ and let us consider an element $\mathscr{V}_{0} \in \mathscr{A}_{0}(R, S)$ and an associated sequence of varifolds $\mathscr{V}_{k}=\mathscr{V}\left(\Sigma_{k}, v_{k}\right)$ complying to (1.5). The estimates of (1.5) and the Cauchy-Schwarz inequality easily imply

$$
\left|\delta \mathscr{V}_{k}(X)\right| \leq K\|X\|_{\infty} \text { for every } X \in \mathscr{D}\left(\mathbf{R}^{d}, \mathbf{R}^{d}\right),
$$

for some constant $K \geq 0$. By the Riesz representation theorem, this implies $\delta \mathscr{V}_{k} \in$ $\mathscr{M}\left(\mathbf{R}^{d}\right)^{d}$ for any $k \geq 1$ with the uniform bound

$$
\left\|\delta \mathscr{V}_{k}\right\|\left(\mathbf{R}^{d}\right) \leq K
$$

We can now state a compactness result due to Hutchinson [9]. It extends Allard's compactness theorem for (non-oriented) integer varifolds [1] to oriented integer varifolds.

Theorem D.1 ([9] Theorem 3.1. ${ }^{1}$ ) Let $K>0$ and let $E_{K}$ be the set of varifolds $\mathscr{V} \in \mathbf{I} \mathbf{V}_{0}^{o}$ such that $\delta \mathscr{V}$ is a Radon measure of $\mathscr{M}\left(\mathbf{R}^{d}\right)^{d}$ and

$$
\|\mathscr{V}\|\left(\mathbf{R}^{d} \times S^{d-1}\right)+\|\delta \mathscr{V}\|\left(\mathbf{R}^{d}\right) \leq K
$$

Then $E_{K}$ is compact in $\mathscr{M}\left(\mathbf{R}^{d} \times S^{d-1}\right)$.

We can apply this result to the sequence $\left(\mathscr{V}_{k}\right)$ and deduce,

$$
\mathscr{V}_{0}=\mathscr{V}\left(\Sigma_{0}, n_{0}, \theta_{0}^{+}, \theta_{0}^{-}\right) \in \mathbf{I} \mathbf{V}_{0}^{o}
$$

We now have to prove that $\mathscr{V}_{0}$ admits a $L^{2}$-generalized mean curvature. Let us introduce this notion.

Definition D.2 ( $L^{2}$-generalized mean curvature. Willmore energy of a varifold)

a) Let $\mathscr{V} \in \mathscr{M}\left(\mathbf{R}^{d} \times S^{d-1}\right)$ and let $\pi \mathscr{V} \in \mathscr{M}\left(\mathbf{R}^{d}\right)$ be the pushforward of $\mathscr{V}$ by the mapping $(y, n) \in \mathbf{R}^{d} \times S^{d-1} \mapsto y \in \mathbf{R}^{d}$, that is $[\pi \mathscr{V}](E)=\mathscr{V}\left(E \times S^{d-1}\right)$ for every Borel set $E \subset \mathbf{R}^{d}$.

We say that $\mathscr{V}$ admits a $L^{2}$-generalized curvature, if there exists $K \geq 0$ such that

$$
\delta \mathscr{V}(X) \leq K\|X\|_{L^{2}\left(\mathbf{R}^{d}, \pi \mathscr{V}\right)} \quad \text { for every } X \in C_{c}^{1}\left(\mathbf{R}^{d}\right)^{d}
$$

Since $\pi \mathscr{V}$ is a Radon measure, it is regular and the space $C_{c}^{1}\left(\mathbf{R}^{d}\right)^{d}$ is dense in $L^{2}\left(\mathbf{R}^{d}, \pi \mathscr{V}\right)^{d}$. Consequently, if we assume the above bound, we can apply the (Hilbert space) Riesz representation theorem to deduce the existence of a $\pi \mathscr{V}$ measurable mapping $H: \operatorname{supp}(\pi \mathscr{V}) \rightarrow \mathbf{R}^{d}$, such that

$$
\delta \mathscr{V}(X)=-\int_{\mathbf{R}^{d}} H \cdot X d[\pi \mathscr{V}] \quad \text { for every } X \in C_{c}^{1}\left(\mathbf{R}^{d}\right)^{d} .
$$

\footnotetext{
1 We only use the special case of oriented integer varifolds without boundary
} 
This formula extends (D.3) and we will say that $H$ is the $\left(L^{2}\right.$-)generalized (vectorial) mean curvature of $\mathscr{V}$.

b) If $\mathscr{V} \in \mathscr{M}\left(\mathbf{R}^{d} \times S^{d-1}\right)$ admits a $L^{2}$-generalized mean curvature $H$, we define its Willmore energy as

$$
\mathscr{W}(\mathscr{V}):=\int_{\mathbf{R}^{d}}|H|^{2} d[\pi \mathscr{V}] .
$$

If $\mathscr{V}$ does not admit a $L^{2}$-generalized mean curvature, we set $\mathscr{W}(\mathscr{V})=+\infty$.

Remark D.2

a) Of course if $\mathscr{V}=\mathscr{V}(\Sigma, v)$, where $\Sigma$ is a smooth compact surface oriented by $v$, then it admits a generalized vectorial mean curvature which matches the usual definition $H=-\left(\operatorname{div}_{\Sigma} v\right) v$. Moreover, $[\pi \mathscr{V}]=\mathscr{H}^{d-1}\llcorner\Sigma$ so $\mathscr{W}(\mathscr{V}(\Sigma, v))=\mathscr{W}(\Sigma)$. b) For $\mathscr{V}=\mathscr{V}\left(\Sigma, v, \theta^{+}, \theta^{-}\right) \in \mathbf{I V}^{o}$, then $[\pi \mathscr{V}]=\left(\theta^{+}+\theta^{-}\right) \mathscr{H}^{d-1}\llcorner\Sigma$. If $\mathscr{V}$ admits a $L^{2}$-generalized mean curvature $H: \Sigma \rightarrow \mathbf{R}^{d}$, then $H$ is $\mathscr{H}^{d-1}$-measurable and we have,

$$
\mathscr{W}(\mathscr{V})=\int_{\Sigma}|H|^{2}\left(\theta^{+}+\theta^{-}\right) d \mathscr{H}^{d-1} .
$$

c) If a rectifiable varifold $\mathscr{V}=\mathscr{V}\left(\Sigma, v, \theta^{+}, \theta^{-}\right)$admits a generalized mean curvature $H$, then $H$ is $[\pi \mathscr{V}]$-almost everywhere collinear to $v$ (see K. Brakke [4]).

It is easily seen that the extension of the Willmore energy defined above is lower semi-continuous with respect to the convergence of Radon measures. Indeed, let $\left(\mathscr{V}_{k}\right) \subset \mathscr{M}\left(\mathbf{R}^{d} \times S^{d-1}\right)$ converging to $\mathscr{V}$ and assume without loss of generality that $\liminf W\left(\mathscr{V}_{k}\right)<\infty$. Using the Cauchy-Schwarz inequality, we have, with obvious notation: for every $X \in C_{c}^{1}\left(\mathbf{R}^{d}\right)^{d}$,

$$
\delta \mathscr{V}_{k}(X)=\left\langle\pi \mathscr{V}_{k} ; H_{k} \cdot X\right\rangle \leq \sqrt{\mathscr{W}\left(\mathscr{V}_{k}\right)}\|X\|_{L^{2}\left(\mathbf{R}^{d}, \pi \mathscr{V}_{k}\right)}=\sqrt{\mathscr{W}\left(\mathscr{V}_{k}\right)} \sqrt{\left\langle\pi \mathscr{V}_{k} ;|X|^{2}\right\rangle}
$$

By the convergence $\mathscr{V}_{k} \rightarrow \mathscr{V}$ as $k \uparrow \infty$, the left hand side converges towards $\delta \mathscr{V}(X)$ and $\|X\|_{L^{2}\left(\mathbf{R}^{d}, \pi^{\mathscr{V}} / k\right)} \rightarrow\|X\|_{L^{2}\left(\mathbf{R}^{d}, \pi^{\mathscr{V}}\right)}$. So,

$$
\delta \mathscr{V}(X) \leq \sqrt{\liminf \mathscr{W}\left(\mathscr{V}_{k}\right)}\|X\|_{L^{2}\left(\mathbf{R}^{d}, \pi \mathscr{V}\right)}
$$

Hence, $\mathscr{V}$ admits a $L^{2}$-generalized vectorial mean curvature $H \in L^{2}\left(\mathbf{R}^{d}, \pi \mathscr{V}\right)^{d}$. Now, by density of $C_{c}^{1}\left(\mathbf{R}^{d}\right)^{d}$ in $L^{2}\left(\mathbf{R}^{d}, \pi \mathscr{V}\right)^{d}$, we have

$$
\sqrt{\mathscr{W}(\mathscr{V})}=\|H\|_{L^{2}\left(\mathbf{R}^{d}, \pi \mathscr{V}\right)}=\sup \langle\mathscr{V} ; X \cdot H\rangle=\sup \delta \mathscr{V}(X),
$$

where the suprema are taken over all $X \in C_{c}^{1}\left(\mathbf{R}^{d}\right)$ such that $\|X\|_{L^{2}\left(\mathbf{R}^{d}, \pi \mathscr{V}\right)} \leq 1$. With (D.6), this yields

$$
\mathscr{W}(\mathscr{V}) \leq \liminf _{k \uparrow \infty} \mathscr{W}\left(\mathscr{V}_{k}\right)
$$

as claimed.

In our context, if $\mathscr{V}_{0} \in \mathscr{A}_{0}$, then it is the limit of a sequence $\mathscr{V}_{k}=\mathscr{V}\left(\Sigma_{k}, v_{k}\right)$ of smooth oriented integer varifolds with uniformly bounded Willmore energy. 
We conclude that $\mathscr{V}_{0}=\mathscr{V}\left(\Sigma_{0}, v_{0}, \theta_{0}^{+}, \theta_{0}^{-}\right) \in \mathbf{I} \mathbf{V}_{0}^{o}$ admits a $L^{2}$-generalized mean curvature $H_{0}$, the formula of Remark D.2.b provides a more explicit expression for $\mathscr{W}\left(\mathscr{V}_{0}\right)$ :

$$
\mathscr{W}\left(\mathscr{V}_{0}\right)=\int_{\Sigma_{0}}\left|H_{0}\right|^{2}\left(\theta_{0}^{+}+\theta_{0}^{-}\right) d \mathscr{H}^{d-1}
$$

Acknowledgements The author thanks the referee for his careful reading of the manuscript and his helpful suggestions concerning the presentation of this paper. The author is grateful to Olivier Pantz for suggesting this research subject and to Simon Masnou for helpful indications on Phase Field models and Varifolds. The author has been partially supported by the Agence National de la Recherche, through the project: ANR-12-BS01-0014-01 Geometrya.

\section{Index of notation}

. ${ }^{\star} \quad$ the superscript $\star$ indicates an ancillary object used in several sections

$G_{\varepsilon}^{\star} \quad \mathbf{1 8}\left(\right.$ Sec.2.4) good set in the construction of $\Sigma_{\varepsilon}$

$U_{\varepsilon}^{\star} \quad \mathbf{1 8}$ (Sec.2.4) bad set in the construction of $\Sigma_{\varepsilon}$

$\beta_{\dot{\phi} 1}^{\star}(\xi, \eta) \quad \mathbf{1 5}$ (Lem.2.4) bound on the energy of "good" balls

$\beta_{\dot{\phi}, 2}^{\star}(\xi) \quad 16$ (Lem.2.5) bound on the energy of "good" balls

$\beta_{\phi, 3}^{\star}(\xi, \eta) \quad 17$ (Lem.2.6) bound on the energy of "good" balls

$\delta_{\not{q}}^{\star} \quad 14$ (Cor.2.1) positive constant, $d\left(\Sigma_{(\varepsilon)}^{s}, F_{(\varepsilon), \delta}\right) \geq \delta_{\dot{q}}^{\star}$ for $s \in(-1 / 2,1 / 2)$

$\Sigma_{\varepsilon}^{\star} \quad \mathbf{1 8}($ Sec. 2.4$) \quad$ defined as $\Sigma_{\varepsilon}^{0} \cap G_{\varepsilon}^{\star}$, good part of $\Sigma_{\varepsilon}$

$\chi^{\star} \quad 4$ (Def.1.1) (non-negative) cut-off function, $\chi^{\star}(1)=1, \operatorname{supp} \chi^{\star} \subset(1 / 2,2)$

$\omega_{\dot{\phi}}^{\star} \quad 14$ (Lem.2.3) a common modulus of continuity for the functions $u_{(\varepsilon)}$

$[\mathscr{P}] \quad 7$ (Sec.1.6) set of elements satisfying the property $\mathscr{P}$

$e^{\perp} \quad 7$ (Sec.1.6) the hyperplane of $\mathbf{R}^{d}$ orthogonal to $e \in S^{d-1}$

$y^{\prime} \quad 7$ (Sec.1.6) the vector $\left(y_{1}, \cdots, y_{d-1}\right) \in \mathbf{R}^{d-1}, y \in \mathbf{R}^{d}$

- $(\varepsilon)$ the subscript $(\varepsilon)$ indicates a rescaled object, in order of appearance:

$$
\begin{aligned}
& t_{(\varepsilon)}, \Omega_{(\varepsilon)} 9 \text { (Sec.2) } \\
& \Omega_{(\varepsilon)}^{\prime}, F_{(\varepsilon)}^{\prime} \mathbf{1 2} \text { (Sec. 2.2) } \\
& O_{(\varepsilon), \delta}, F_{(\varepsilon), \delta} 12 \text { (Sec.2.2) } \\
& u_{(\varepsilon)} 13 \text { (Def.2.1) } \\
& \Sigma_{(\varepsilon)}^{s}, n_{(\varepsilon)} 13 \text { (Def.2.2) } \\
& U_{(\varepsilon)}^{\star}, G_{(\varepsilon)}^{\star} 18 \text { (Sec. 2.4) }
\end{aligned}
$$

$\mathscr{A}_{\varepsilon} \quad 2$ set of admissible configurations

$\mathscr{A}_{\varepsilon}(R) \quad 2$ set of admissible configurations supported in $B_{R}$

$\mathscr{A}_{\varepsilon}(R, S) 2$ configurations of $\mathscr{A}_{\varepsilon}(R)$ with total mass $2 \varepsilon S$

$\mathscr{A}_{0}(R, S) \mathbf{5}$ (Def.1.3) limit set, subset of $\mathscr{M}\left(\mathbf{R}^{d} \times S^{d-1}\right)$

a 3 triplet $(\sigma, \tau, \Omega)$, a generic configuration of $\mathscr{A}_{\varepsilon}$

$a_{\varepsilon} \quad \mathbf{6}$ (Thm.1.1), $\mathbf{6}$ (Thm.1.2) triplet $\left(\sigma_{\varepsilon}, \nabla t_{\varepsilon}, \Omega_{\varepsilon}\right) \in \mathscr{A}_{\varepsilon}(R, S)$

$B_{r}(y) \quad 7$ (Sec.1.6) open ball with center $y$ and radius $r$ in $\mathbf{R}^{d}, B_{r}=B_{r}(0)$

$C\left(\alpha_{1}, \cdots, \alpha_{k}\right) \mathbf{7}$ (Sec.1.6) a (non-negative) constant only depending on the dimension $d$ and on the parameters $\alpha_{1}, \cdots, \alpha_{k}$ 
$C_{\mathscr{q}}\left(\alpha_{1}, \cdots, \alpha_{k}\right) 7$ (Sec.1.6) a constant only depending on the parameters $\alpha_{1}, \cdots, \alpha_{k}$ and on the data introduced in the hypotheses $\left(d, R, S, \omega, \chi^{\star}\right.$ or $\left.E_{0}\right)$ but not on $\varepsilon$

$c_{0}(f) \quad \mathbf{6}$ (Thm.1.2) positive number depending on the Hessian matrix of $f$

$c_{\xi, \eta, \alpha}(f) \quad 39$ (Def.4.3) minimum value of the local optimization problem

$D_{\lambda}^{\prime}(\bar{n}) \quad 15$ (Def.2.4) a $(d-1)$-ball in $\bar{n}^{\perp}$ with center 0 and radius $\lambda, D_{\lambda}^{\prime}=D_{\lambda}^{\prime}\left(e_{d}\right)$

$D_{\lambda}^{\xi}(\bar{n}) \quad 15\left(\right.$ Def.2.4) cylinder with basis $D_{\lambda}^{\prime}(\bar{n})$ and width $2(1-\xi), D_{\lambda}^{\xi}=D_{\lambda}^{\xi}\left(e_{d}\right)$

$d \quad$ dimension of the ambient space, $d \geq 2$

$d(A, B)$ the (euclidian) distance between the point (or set) $A$ and the set $B$

$\mathscr{E}_{(\varepsilon)}, \mathscr{E}_{(\varepsilon), \lambda} 15$ (Def.2.3) local energy

$\mathscr{E}^{\#}\left(a^{\#}\right) \quad 39$ (Def.4.3) part of the energy of a local configuration $a^{\#}$

$E_{0} \quad 4$ (Eq.1.4) bound on the energy $\mathscr{F}_{0}\left(a_{\varepsilon}\right) / \varepsilon^{3}$

$\left(e_{1}, \cdots, e_{d}\right)$ standard basis of $\mathbf{R}^{d}$

$\mathscr{F}(a) \quad \mathbf{3}$ (Sec.1.1) elastic energy of a configuration $a$

$\mathscr{F}_{0}(a) \quad \mathbf{3}$ (Sec.1.1) energy of the configuration $a$ with the stored energy function $f_{0}$

$\mathscr{F}_{(\varepsilon), \alpha} 38$ (Sec.4.2) local energy

$\mathscr{F}_{\alpha}^{\#}\left(a^{\#}\right) \quad 39$ (Def.4.3) energy of a local configuration $a^{\#}$

$f \quad 3$ (Eq.1.2) stored energy function

$f_{0} \quad \mathbf{3}$ (Eq.1.2) archetype of a non-degenerate stored energy function

$\mathscr{H}^{k}(E) \quad 7$ (Sec.1.6) $\quad k$-dimensional Hausdorff measure of the set $E \subset \mathbf{R}^{d}$

$h_{\varepsilon} \quad$ mean curvature of the hypersurface $\Sigma_{\varepsilon}$

$\hat{h}_{\varepsilon} \quad 32$ (Def.4.2) approximate mean curvature of the hypersurface $\Sigma_{\varepsilon}$

$\hat{h}^{\#}\left(a^{\#}\right) \quad 39$ (Def.4.3, Eq.5.7) approximate curvature of the local configuration $a^{\#}$

$L \quad 6$ (Thm.1.2) $2 \times 2$ matrix extracted from $D^{2} f\left(e_{d}, e_{d}\right)$

$\mathscr{M}\left(\mathbf{R}^{d} \times S^{d-1}\right) 4$ (Def.1.1) space of Radon measures over $\mathbf{R}^{d} \times S^{d-1}$

$M_{\varepsilon} \quad \mathbf{9}$ (Prop.2.1) the set $\left[\left|t_{\varepsilon}\right|<\varepsilon\right]$

$\mathscr{N} \quad \mathbf{6}$ (Eq.1.6), $\mathbf{6}$ (Eq.1.7) a neighbourhood of the identity matrix in $\mathbf{R}^{d \times d}$

$n_{\varepsilon} \quad 13$ (Def.2.2) $\quad \nabla u_{\varepsilon} /\left|\nabla u_{\varepsilon}\right|$ whenever it makes sense

$O_{\varepsilon} \quad \mathbf{9}$ (Prop.2.1) smooth open set approximating $M_{\varepsilon}=\left[t_{\varepsilon}<0\right]$

$\mathscr{Q}(\sigma, \tau) \quad 2$ quantity of material

$S^{d-1} \quad 7$ (Sec.1.6) $(d-1)$-dimensional unit sphere of $\mathbf{R}^{d}$

$\mathbb{S}^{d-1} \quad \mathbf{3}$ the set of zeros of the stored energy functions - a $(d-1)$-dimensional unit sphere embedded in $\mathbf{R}^{d} \times \mathbf{R}^{d}$

$R \quad 2,5$ (Def.1.3), $\mathbf{6}$ (Th.1.1), $\mathbf{6}$ (Th.1.2) confining radius in the definition of $\mathscr{A}_{\varepsilon}(R)$ and $\mathscr{A}_{\varepsilon}(R, S)$

$S \quad 2,5$ (Def.1.3), 6 (Th.1.1), 6 (Th.1.2) "area" of the configurations of $\mathscr{A}_{\mathcal{E}}(R, S)$ and of the elements of $\mathscr{A}_{0}(R, S)$

$T_{\mathcal{E}} \quad \mathbf{6}(\mathrm{Thm} .1 .1), \mathbf{6}(\mathrm{Thm} .1 .2) \quad$ approximate characteristic function of $\left[t_{\varepsilon}<0\right]$, defined as $T_{\varepsilon}:=\left(\varepsilon-t_{\varepsilon}\right) / 2 \varepsilon$

$u_{\varepsilon} \quad 13$ (Def.2.1) harmonic extension of $t_{\varepsilon}$

$\mathscr{V} \quad 4$ (Def.1.1) a generic oriented $(d-1)$-varifold

$\mathscr{V}(\Sigma, v) \quad 4$ (Def.1.1) varifold associated with the oriented hypersurface $(\Sigma, v)$

$\mathscr{V}_{\varepsilon}^{\star}(a) \quad \mathbf{5}$ (Def.1.2) varifold associated with the configuration $a$

$\mathscr{V}_{0} \quad \mathbf{6}$ (Thm.1.1), 6 (Thm.1.2) limit varifold

$\mathscr{W}(\Sigma) \quad 2$ Willmore energy of the hypersurface $\Sigma$

$W_{l o c}^{1,2}\left(\mathbf{R}^{d}\right)$ Sobolev space of functions with locally square integrable derivatives, endowed with its standard topology 
$\zeta, \zeta_{/ /}, \zeta_{\perp} \mathbf{3 1}$ (Def.4.1) test functions for the definition of $\hat{h}_{\varepsilon}$

$\kappa 3$ (Eq.1.2) positive constant introduced in the hypothesis $f \geq \kappa f_{0}$

$\Lambda \mathscr{V} \quad 4$ (Def.1.1) distribution associated with the oriented $(d-1)$-varifold $\mathscr{V}$

$v_{\varepsilon} \quad 9$ (Prop.2.1) outward unit normal to $\Sigma_{\varepsilon}=\partial O_{\varepsilon}$

$\pi_{e} \quad 7$ (Sec.1.6) the projection onto the hyperplane orthogonal to $e \in S^{d-1}$

$\Sigma_{\varepsilon} \quad 9$ (Prop.2.1), 17 (Sec.2.4) smooth hypersurface, the boundary of $O_{\varepsilon}$

$\Sigma_{\varepsilon}^{s} \quad \mathbf{1 3}$ (Def.2.2) level sets of $u_{\varepsilon}$

$\chi_{\varepsilon, \xi} \quad 23$ (Sec.2.5) cut-off function in the proof of Prop. 2.1.c

$\chi_{\varepsilon, \eta} \quad 32$ (Def.4.2) cut-off function for the definition of $\hat{h}_{\varepsilon}$

$\chi_{\varepsilon, \eta}^{\prime} \quad 34$ (Sec.4.1) cut-off function in the proof of Prop. 4.1

$\omega \quad 3$ (Hyp.1) a common modulus of continuity for the functions $t_{(\varepsilon)}$

\section{References}

1. William K. Allard. On the first variation of a varifold. Ann. of Math. (2), 95:417-491, 1972.

2. Frederick J. Almgren. The theory of varifolds. Mimeographed lecture. Princeton Univ. Press, Princeton, 1965.

3. Luigi Ambrosio, Nicola Fusco, and Diego Pallara. Functions of bounded variation and free discontinuity problems. Oxford Mathematical Monographs. The Clarendon Press Oxford University Press, New York, 2000.

4. Kenneth A. Brakke. The motion of a surface by its mean curvature, volume 20 of Mathematical Notes. Princeton University Press, Princeton, N.J., 1978.

5. Lawrence C. Evans and Ronald F. Gariepy. Measure theory and fine properties of functions. Studies in Advanced Mathematics. CRC Press, Boca Raton, FL, 1992.

6. Gero Friesecke, Richard D. James, and Stefan Müller. A theorem on geometric rigidity and the derivation of nonlinear plate theory from three-dimensional elasticity. Comm. Pure Appl. Math., 55(11):1461-1506, 2002.

7. David Gilbarg and Neil S. Trudinger. Elliptic partial differential equations of second order. Classics in Mathematics. Springer-Verlag, Berlin, 2001. Reprint of the 1998 edition.

8. Wolfgang Helfrich. Elastic properties of lipid bilayers-theory and possible experimentsl. $Z$. Naturforsch, C28:693-703, 1973.

9. John E. Hutchinson. Second fundamental form for varifolds and the existence of surfaces minimising curvature. Indiana Univ. Math. J., 35(1):45-71, 1986.

10. Ulrich Menne. Second Order Rectifiability of Integral Varifolds of Locally Bounded First Variation. J. Geom. Anal., 23(2):709-763, 2013.

11. Benoît Merlet. A highly anisotropic nonlinear elasticity model for vesicles. I: Eulerian formulation, rigidity estimates and vanishing energy limit. Submitted, pages 1-23, 2013.

12. Reiner Schätzle. Quadratic tilt-excess decay and strong maximum principle for varifolds. Ann. Sc. Norm. Super. Pisa Cl. Sci. (5), 3(1):171-231, 2004.

13. Leon Simon. Lectures on geometric measure theory, volume 3 of Proceedings of the Centre for Mathematical Analysis, Australian National University. Australian National University Centre for Mathematical Analysis, Canberra, 1983.

14. Leon Simon. Existence of surfaces minimizing the Willmore functional. Comm. Anal. Geom., 1(2):281-326, 1993. 